\title{
THE SCHWARTZ SPACE OF A GENERAL SEMISIMPLE LIE GROUP. II: WAVE PACKETS ASSOCIATED TO SCHWARTZ FUNCTIONS
}

\author{
REBECCA A. HERB
}

\begin{abstract}
Let $G$ be a connected semisimple Lie group. If $G$ has finite center, Harish-Chandra used Eisenstein integrals to construct Schwartz class wave packets of matrix coefficients and showed that every $K$-finite function in the Schwartz space is a finite sum of such wave packets. This paper is the second in a series which generalizes these results of Harish-Chandra to include the case that $G$ has infinite center. In this paper, the Plancherel theorem is used to decompose $K$-compact Schwartz class functions (those with $K$-types in a compact set) as finite sums of wave packets. A new feature of the infinite center case is that the individual wave packets occurring in the decomposition of a Schwartz class function need not be Schwartz class. These wave packets are studied to obtain necessary conditions for a wave packet of Eisenstein integrals to occur in the decomposition of a Schwartz class function. Applied to the case that $f$ itself is a single wave packet, the results of this paper yield a complete characterization of Schwartz class wave packets.
\end{abstract}

\section{INTRODUCTION}

Suppose $G$ is a connected semisimple Lie group. Then the tempered spectrum of $G$ consists of families of representations induced unitarily from cuspidal parabolic subgroups. Each family is parametrized by the unitary characters of a Cartan subgroup. The Plancherel theorem expands Schwartz class functions on $G$ in terms of the distribution characters of these tempered representations. Very roughly, for $f$ in the Schwartz space $\mathscr{C}(G)$ we can write

$$
f(x)=\sum f_{H}(x), \quad H \in \operatorname{Car}(G),
$$

where $\operatorname{Car}(G)$ denotes a complete set of representatives for conjugacy classes of Cartan subgroups of $G$ and

$$
f_{H}(x)=\int_{\widehat{H}} \Theta(H: \chi)(R(x) f) m(H: \chi) d \chi .
$$

Here $\Theta(H: \chi)$ denotes the distribution character of the representation $\pi(H: \chi)$

Received by the editors June 27, 1989.

1980 Mathematics Subject Classification (1985 Revision). Primary 22E30, 22E46.

The author was partially supported by NSF Grant DMS-88-02586. 
corresponding to $\chi \in \widehat{H}, R(x) f$ is the right translate of $f$ by $x \in G$, and $m(H: \chi) d \chi$ is the Plancherel measure. (See [5] for details on the Plancherel theorem.)

Suppose $G$ has finite center and $f$ is $K$-finite, where $K$ is a maximal compact subgroup of $G$. Fix $H \in \operatorname{Car}(G)$. Then for each $\chi \in \widehat{H}, \Theta(H: \chi)(R(x) f)$ is a finite sum of $K$-finite matrix coefficients of the representation $\pi(H: \chi)$. Thus we can think of $f_{H}$ as a wave packet of matrix coefficients associated to $f$. In $[1,2,3]$ Harish-Chandra used Eisenstein integrals to construct Schwartz class wave packets of matrix coefficients. He then showed that for every $K$ finite $f \in \mathscr{C}(G), f_{H}$ is a finite sum of wave packets of the type constructed. In particular, this shows that $f_{H} \in \mathscr{C}(G)$.

Now suppose that $G$ has infinite center $Z_{G}$. Let $K$ be a maximal relatively compact subgroup. (That is, $K / Z_{G}$ is compact.) There are no $K$-finite functions in $\mathscr{C}(G)$. However the set $\mathscr{C}(G)_{K}$ of $K$-compact functions, those with $K$-types lying in a compact subset of $\widehat{K}$, is dense in $\mathscr{C}(G)$. (See Theorem 2.12 of this paper.) Now for any $\pi \in \widehat{G}$, the $K$-types of $\pi$ lie in a discrete subset of $\widehat{K}$ since they have a fixed $Z_{G}$-character. Thus for any $\chi \in \widehat{H}$ and $f \in \mathscr{C}(G)_{K}, \Theta(H: \chi)(R(x) f)$ is a finite sum of $K$-finite matrix coefficients of the representation $\pi(H: \chi)$. Thus again we can think of $f_{H}$ as a wave packet of matrix coefficients.

The purpose of this paper is to study these wave packets associated to Schwartz class functions. A new feature of the infinite center case is that for $f \in \mathscr{C}(G), f_{H}$ is not necessarily Schwartz class. This is because of interference between different series of representations when a principal series representation decomposes as a sum of limits of discrete series. When $G$ has infinite center, these limits of discrete series are actual limits along continuous families of relative discrete series representations, and so occur in a nontrivial way in the Plancherel formula in the terms corresponding to different Cartan subgroups. This means that, for $f \in \mathscr{C}(G)$, there are matching conditions between the terms $f_{H}, H \in \operatorname{Car}(G)$, which are necessary in order that the sum be a Schwartz function when the individual terms are not. These matching conditions are derived from a character identity (Theorem 9.6) that generates that of W. Schmid [12] to include derivatives of all orders along the continuous parameters. Thus at each point where two continuous series meet there are infinitely many derivatives of the two associated families of matrix coefficients that must match if the sum of the wave packets is to be a Schwartz function.

It has been known for some time that matching conditions would be necessary for Schwartz functions in the infinite center case. This was first pointed out to me in 1984 by D. Miličić. He and H. Kraljević discovered this phenomenon when they were studying the Fourier transform for the $C^{*}$-algebra of the universal covering group of $S L(2, \mathbf{R})[10]$.

In [9], the author, together with J. A. Wolf, defined Eisenstein integrals and used them to form Schwartz class wave packets. These, as in the finite center case, are formed from a single continuous family of representations, although in 
the infinite center case there are extra continuous parameters coming from continuous families of relative discrete series representations. Thus the Schwartz functions constructed lie in $\mathscr{C}_{H}(G)=\left\{f \in \mathscr{C}(G): f=f_{H}\right\}$, and these wave packets must satisfy conditions to make them match with the functions $f_{H^{\prime}}=0$ for other Cartan subgroups $H^{\prime}$. In fact, one of the conditions assumed was too strong. In [4], the results of [9] are improved by analyzing the behavior of $c$ functions more carefully as a function of all continuous parameters. Less restrictive conditions than those used in [9] are shown to be sufficient for a single series wave packet to be a Schiwartz function.

In this paper it is shown that the conditions assumed in [4] are necessary for a wave packet of Eisenstein integrals to be a Schwartz class function. Thus the results of this paper, combined with those of $[4,9]$, give a complete characterization of the $K$-compact functions in $\mathscr{C}_{H}(G)$. Conditions are also given which are necessary for a wave packet of Eisenstein integrals to be a wave packet associated to a Schwartz class function, that is a wave packet which may not be Schwartz, but which can be patched together with wave packets from other series of representations to form a Schwartz class function. These necessary conditions are obtained by studying the properties of the wave packets associated to functions in $\mathscr{C}_{H}(G)$ and $\mathscr{C}(G)$, respectively.

To explain the main results of this paper more precisely, we need some notation. Let $G$ be a connected reductive Lie group and let $K$ be a maximal relatively compact subgroup of $G$. It is proved in [9] that $K=K_{1} \times V$, where $K_{1}$ is compact and $V$ is a vector group. Now $\widehat{V}=\left\{e^{h}: h \in i^{*}\right\}$ is a vector group and $\widehat{K}$ is the union of continuous families $\left\{\tau \otimes e^{h}: h \in i v^{*}\right\}$, where $\tau \in \widehat{K}$. Now let $P=M A N$ be a cuspidal parabolic subgroup of $G$ and $H=T \times A$ a Cartan subgroup with $T \subseteq K$ a maximal relatively compact Cartan subgroup of $M$. The characters $e^{\bar{h}}$ of $K$ give characters of $T$ by restriction. Thus the characters of $T$ lie in continuous families of the form $\left\{\chi \otimes e^{h}: h \in i v^{*}\right\}$, $\chi \in \widehat{T}$. (These are not all distinct characters of $T$.) Each character in the family corresponds to a relative discrete series or limit of relative discrete series representation $\pi(M: h)$. Let $\mathscr{D}$ be a convex open subset of $i v^{*}$ so that the Harish-Chandra parameters of the corresponding representations lie in a fixed Weyl chamber and points on the boundary of $\mathscr{D}$ correspond to limits of discrete series. Then a continuous family of representations corresponding to $H$ will be of the form $\left\{\pi(H: h: \nu)=\operatorname{Ind}_{M A N}^{G}(\pi(M: h) \otimes \nu \otimes 1): h \in \mathscr{D}, \nu \in \widehat{A}\right\}$. It is proved in [6] that for fixed $\tau \in \widehat{K}$, the multiplicity of $\tau \otimes e^{h}$ in $\pi(H: h: \nu)$ is independent of $h \in \mathscr{D}, \nu \in \hat{A}$.

Fix a continuous family $\{\pi(H: h: \nu): h \in \mathscr{D}, \nu \in \widehat{A}\}$ as above and $\tau_{1}, \tau_{2} \in \widehat{K}$ such that the $\tau_{i} \otimes e^{h}$ occur in $\pi(H: h: \nu), i=1,2$. For any $f \in \mathscr{C}(G)$, define

$$
\hat{f}(h: \nu: x)=\delta_{1}^{*}(h){ }_{K / Z} \Theta(H: h: \nu)(R(x) f){ }_{K / Z} \delta_{2}(h),
$$

where $\delta_{1}^{*}(h)$ and $\delta_{2}(h)$ are the normalized characters of $\tau_{1} \otimes e^{h}$ and $\left(\tau_{2} \otimes e^{h}\right)^{*}$ 
respectively, and $\Theta(H: h: \nu)$ is the distribution character of $\pi(H: h: \nu)$. Then set

$$
f(H: x)=\int_{\mathscr{D} \times \widehat{A}} \hat{f}(h: \nu: x) m(h: \nu) d h d \nu,
$$

where $m(h: \nu) d h d \nu$ is Plancherel measure. Then $f(H: x)$ is called a wave packet corresponding to $f$. Theorem 3.5 of this paper says that if $f \in \mathscr{C}(G)$ is $K$-compact, then $f$ is a finite sum of wave packets of this type.

The wave packets of Eisenstein integrals in [4, 9] were defined as follows. First we defined Eisenstein integrals $E(h: \nu)$ which are smooth families of $K$ finite matrix coefficients of the representations $\pi(H: h: \nu)$. Then we defined wave packets of the form

$$
f_{\alpha}(x)=\int_{\mathscr{D} \times \widehat{A}} E(h: \nu: x) \alpha(h: \nu) m(h: \nu) d h d \nu,
$$

where $\alpha$ is a jointly smooth function of $h$ and $\nu$ which is rapidly decaying at infinity in both variables. We proved that $f_{\alpha}$ is a Schwartz class function if $\alpha$ is also rapidly decaying as $h$ approaches the boundary of $\mathscr{D}$ and $E(h: \nu: x) \alpha(h: \nu) m(h: \nu)$ is jointly smooth. This second condition is a restriction only at points where $m$, which is separately smooth in $h$ and $\nu$, fails to be jointly smooth.

Theorem 8.3 of this paper says that every wave packet $f(H: x)$ corresponding to $f \in \mathscr{C}(G)$ as in $(1.2 \mathrm{~b})$ is a finite sum of wave packets $f_{\alpha}$ of Eisenstein integrals as defined in (1.3). Moreover, if $f \in \mathscr{C}_{H}(G)$, then the functions $\alpha$ are rapidly decaying as $h$ approaches the boundary of $\mathscr{D}$. Theorem 10.22 gives matching conditions between $\hat{f}(h: \nu: x)$ and corresponding factors from other series of representations which must be satisfied for any $f \in \mathscr{C}(G)$. Theorem 10.24 says that if $f \in \mathscr{C}_{H}(G)$, then $\hat{f}(h: \nu: x) m(h: \nu)$ is jointly smooth. Points where $m(h: \nu)$ is not jointly smooth correspond to principal series representations which decompose into sums of limits of discrete series which occur as actual limits along continuous families of relative discrete series. The fact that $\hat{f}(h: \nu: x)$ matches with zero at these points implies that $\hat{f}(h: \nu: x) m(h: \nu)$ is jointly smooth.

In order to complete the characterization of the $K$-compact functions in the Schwartz space for the infinite center case it is necessary to prove that the matching conditions coming from character formulas are sufficient to guarantee that a finite sum of non-Schwartz wave packets is Schwartz. This will be shown in another paper.

In this paper $G$ is always assumed to be a connected reductive Lie group. A larger class of reductive groups was defined by Wolf in [15]. This larger class includes the Levi components $M$ of all cuspidal parabolic subgroups $P=$ $M A N$ of $G$. However, in [4, 9] and in this paper results are not proved by induction on the dimension of $G$, and so it is not necessary to work inside a class of groups closed under passing from $G$ to $M$. It was shown in [9] that 
every group in the larger class is obtained as follows. Let $G^{0}$ be the identity component of $G$. Then $G$ has a closed normal subgroup of finite index of the form $G^{\prime}=G^{0} \times E$, direct product, where $E$ is a finitely generated free abelian group. In [9] we indicated how results on wave packets of matrix coefficients for $G^{0}$ could be extended first to $G^{\prime}$ and then to $G$. It would be a routine exercise to use the same techniques to extend the results of this paper to this class of groups.

The organization of the paper is as follows.

In $\S 2$, the definition of $\mathscr{C}(G)$ is reviewed and the set $\mathscr{C}(G)_{K}$ of $K$-compact Schwartz functions is defined. The main result is Theorem 2.12 which says that $\mathscr{C}(G)_{K}$ is dense in $\mathscr{C}(G)$.

In $\S 3$, the parametrization of continuous families of tempered representations is reviewed and the wave packets corresponding to $f \in \mathscr{C}(G)$ are defined. The main result is Theorem 3.5 which says that each $f \in \mathscr{C}(G)_{K}$ is a finite sum of these wave packets.

In $\S 4$, the families $\Psi_{f}(h: \nu)$ of spherical functions of matrix coefficients of the relative discrete series representations $\pi(M: h)$ are defined corresponding to $f \in \mathscr{C}(G)$. The main result is Theorem 4.6 which says that the Fourier transform functions $\hat{f}(h: \nu)$ defined in (1.2a) can be expressed in terms of Eisenstein integrals of these spherical functions.

In $\S 5$, vector spaces $\mathscr{S}(h)$ of spherical functions of matrix coefficients of the $\pi(M: h)$ are studied. The main result is Theorem 5.32 which shows how to construct bases for $\mathscr{S}(h)$ and its dual $\mathscr{S}(h)^{*}$, which vary smoothly as $h$ varies. This is a very technical section and the results are proved using the universal cover $M^{\prime}$ of $M^{0}$, the identity component of $M$.

The main result of $\S 6$ is Theorem 6.1, which gives a growth estimate for the spherical functions $\Psi_{f}(h: \nu)$ associated to $f \in \mathscr{C}(G)$. In Theorem 7.2 this estimate is sharpened for the case $f \in \mathscr{C}_{H}(G)$.

The results of $\S \S 5-7$ are combined in $\S 8$ (Theorem 8.3 ) to show that every wave packet associated to $f \in \mathscr{C}(G)$ is a finite sum of wave packets $f_{\alpha}$ of Eisenstein integrals. Further, if $f \in \mathscr{C}_{H}(G)$, the functions $\alpha$ are shown to decay at the boundary of $\mathscr{D}$.

In $\S 9$ (Theorem 9.6), the character identity of Schmid is generalized to include derivatives along the continuous families of parameters.

Finally in $\S 10$, the Plancherel function $m(h: \nu)$ is studied. In Theorem 10.22 , the character formula of $\S 9$ is used to prove matching conditions for the functions $\hat{f}(h: \nu)$ associated to $f \in \mathscr{C}(G)$. These matching conditions are used in Theorem 10.24 to show that if $f \in \mathscr{C}_{H}(G)$, then $\hat{f}(h: \nu) m(h: \nu)$ is jointly smooth.

This paper is a continuation of a study of harmonic analysis for semisimple Lie groups with infinite center which has been joint work with J. A. Wolf (see [5-9]). I am grateful to him for many suggestions and helpful comments during the writing of this paper. In particular, he provided the proofs for Lemmas 6.6-6.8. 


\section{The SchwartZ SPACE AND $K$-COMPaCt fUNCTIONS}

Suppose $G$ is a connected reductive Lie group. Fix a Cartan involution $\theta$ of $G$ as in [15] and let $K$ denote the fixed point set of $\theta$. It is the full inverse image of a maximal compact subgroup of the linear group $G / Z_{G}$. The following was proved in [9].

Proposition 2.1. $K$ has a unique maximal compact subgroup $K_{1}$ and has a closed normal vector subgroup $V$ such that

(a) $K=K_{1} \times V$,

(b) $Z=Z_{G} \cap V$ is co-compact in both $V$ and $Z_{G}$.

Let $\mathfrak{g}=\mathfrak{k}+\mathfrak{p}$ be the \pm 1 eigenspace decomposition under $\theta$. Choose a maximal abelian subspace $\mathfrak{a}_{0} \subset \mathfrak{p}$ and a positive restricted root system $\Phi^{+}=$ $\Phi^{+}\left(\mathfrak{g}, \mathfrak{a}_{0}\right)$. As usual, $\rho=\frac{1}{2} \sum_{\alpha \in \Phi^{+}} m(\alpha) \alpha$, where $m(\alpha)=\operatorname{dim} \mathfrak{g}_{\alpha}$. The Iwasawa decomposition

$$
G=N_{0} A_{0} K, \quad x=n(x) \cdot \exp H(x) \cdot \kappa(x)
$$

specifies the zonal spherical function on $G$ for $0 \in \mathfrak{a}_{0}^{*}$,

$$
\Xi(x)=\int_{K / Z} e^{-\rho(H(k x))} d(k Z) .
$$

Growth in $G$ is determined by a function $\tilde{\sigma}: G \rightarrow \mathbf{R}^{+}$which is defined as follows. Choose an $\operatorname{Ad}_{G}(K)$-invariant positive definite inner product on $V$. If $x \in G$ we decompose

$$
x=v(x) \cdot k_{1}(x) \cdot \exp \xi(x) \in V K_{1} \cdot \exp (\mathfrak{p})
$$

and then we set

$$
\sigma_{V}(x)=\|v(x)\|, \quad \sigma(x)=\|\xi(x)\|, \quad \tilde{\sigma}(x)=\sigma_{V}(x)+\sigma(x) .
$$

The main properties of $\sigma$ are

$$
\sigma\left(k_{1} x k_{2}\right)=\sigma(x) \text { for all } x \in G, k_{1}, k_{2} \in K
$$

and

$$
\sigma(x y) \leq \sigma(x)+\sigma(y) \text { for all } x, y \in G .
$$

The corresponding properties of $\tilde{\sigma}$ are

$$
\begin{gathered}
\tilde{\sigma}\left(k x k^{-1}\right)=\tilde{\sigma}(x) \quad \text { for all } x \in G, k \in K \\
\tilde{\sigma}\left(k_{1} x k_{2}\right)=\tilde{\sigma}(x) \text { for all } x \in G, k_{1}, k_{2} \in K_{1} ; \\
\tilde{\sigma}(x y) \leq 3(\tilde{\sigma}(x)+\tilde{\sigma}(y)) \text { for all } x, y \in G .
\end{gathered}
$$

Let $W$ be a Banach space and $f \in C^{\infty}(G: W)$. If $D_{1}, D_{2} \in \mathscr{U}(\mathfrak{g})$ and $r \in \mathbf{R}$ we define

$$
D_{1}\|f\|_{r, D_{2}}=\sup _{x \in G}(1+\tilde{\sigma}(x))^{r} \Xi(x)^{-1}\left\|f\left(D_{1} ; x ; D_{2}\right)\right\|_{W} .
$$


The Schwartz space is

$$
\begin{aligned}
\mathscr{C}(G: W)=\left\{f \in C^{\infty}(G: W):\right. & \|f\|_{r, D_{2}}<\infty \\
& \text { for all } \left.D_{1}, D_{2} \in \mathscr{U}(\mathfrak{g}) \text { and all } r \in \mathbf{R}\right\} .
\end{aligned}
$$

It is a complete locally convex topological vector space with the topology defined by the seminorms $(2.8 \mathrm{a})$. And of course the most important case is

$$
\mathscr{C}(G)=\mathscr{C}(G: \mathbf{C}) \text {. }
$$

For any $\zeta \in \hat{Z}$, let $C^{\infty}(G / Z, \zeta)=\left\{f \in C^{\infty}(G): f(x z)=\overline{\zeta(z)} f(x)\right.$ for all $z \in Z, x \in G\}$. Then we also have the relative Schwartz spaces

$$
\begin{array}{r}
\mathscr{C}(G / Z, \zeta)=\left\{f \in C^{\infty}(G / Z, \zeta): \sup (1+\sigma(x))^{r} \Xi(x)^{-1}\left|f\left(D_{1} ; x ; D_{2}\right)\right|<\infty\right. \\
\text { for all } \left.D_{1}, D_{2} \in \mathscr{U}(\mathfrak{g}), \quad r \in \mathbf{R}\right\} .
\end{array}
$$

For $f \in \mathscr{C}(G), \zeta \in \widehat{Z}$, define

$$
f_{\zeta}(x)=\int_{Z} f(x z) \zeta(z) d z
$$

Then it was proved in $[6,7.2]$ that $f_{\zeta} \in \mathscr{C}(G / Z, \zeta)$ for all $f \in \mathscr{C}(G), \zeta \in \widehat{Z}$.

When $K$ is noncompact, there are no $K$-finite functions in $\mathscr{C}(G)$. We replace the notion of $K$-finiteness by $K$-compactness, which is equivalent when $K$ is compact so that $\widehat{K}$ is discrete. For $\tau \in \widehat{K}$, let

$$
\delta(\tau)=\operatorname{deg} \tau^{*} \operatorname{tr} \tau^{*}
$$

denote the normalized character of the contragradient $\tau^{*}$ of $\tau$. We say $f \in$ $\mathscr{C}(G)$ is $K$-compact if there is a compact subset $\Omega$ of $\widehat{K}$ so that

$$
\delta\left(\tau^{*}\right){ }_{K} f=0=f{ }^{*}{ }_{K} \delta(\tau) \quad \text { unless } \tau \in \Omega .
$$

Write $\mathscr{C}(G)_{K}$ for the set of all $K$-compact functions in $\mathscr{C}(G)$ and let $\mathscr{C}_{H}(G)=$ $\left\{f \in \mathscr{C}(G): f=f_{H}\right\}$ when $f_{H}$ is defined as in (1.1b).

Theorem 2.12. $\mathscr{C}(G)_{K}$ is a dense subspace of $\mathscr{C}(G)$. Further, for any $H \in$ $\operatorname{Car}(G), \mathscr{C}_{H}(G)_{K}=\mathscr{C}_{H}(G) \cap \mathscr{C}(G)_{K}$ is a dense subspace of $\mathscr{C}_{H}(G)$.

The remainder of this section is devoted to the proof of Theorem 2.12. First we state a series of lemmas. The arguments are an elementary generalization of those of $[8, \S 12]$, so the proofs are omitted.

Let $G_{1}=\left\{x \in G: x=k \exp (\xi)\right.$ for some $k \in K_{1}$ and $\left.\xi \in \mathfrak{p}\right\}$. Then using $K=K_{1} \times V$ we can write every $x \in G$ uniquely as $x=v x_{1}$, where $v \in V$ and $x_{1} \in G$. Further, by (2.5), $\tilde{\sigma}(x)=\sigma_{V}(x)+\sigma\left(x_{1}\right)$. For $f \in C^{\infty}(G), x \in G$, $v \in V$, define

$$
f(v: x)=f(v x)
$$


For $D, D^{\prime} \in \mathscr{U}(\mathfrak{g}), b \in \mathscr{U}(\mathfrak{v}), r, s \geq 0$, define

$\mu\left(D, D^{\prime}, b, r, s\right)(f)=\sup _{\substack{x \in G_{1} \\ v \in V}} \Xi^{-1}(x)(1+\sigma(x))^{r}\left(1+\sigma_{V}(v)\right)^{s}\left|f\left(v ; b: D_{c} x ; D^{\prime}\right)\right|$,

$$
\mathscr{C}(G)^{\prime}=\left\{f \in C^{\infty}(G): \mu\left(D, D^{\prime}, b, r, s\right)(f)<\infty \text { for all } D, D^{\prime}, b, r, s\right\} .
$$

Lemma 2.14. $\mathscr{C}(G)^{\prime}=\mathscr{C}(G)$ and the families of seminorms $\left\{_{D_{1}}\|\cdot\|_{r, D_{2}}\right\}$ and $\left\{\mu\left(D, D^{\prime}, b, r, s\right)\right\}$ are equivalent.

Proof. See [8, Lemma 12.13].

Let $\mathscr{C}\left(i v^{*}\right)$ denote the ordinary Euclidean Schwartz space for $i v^{*}$. Similarly, define $\mathscr{C}(V)$ to be the Schwartz space for the vector group $V$ using the seminorms

(2.15a)

$$
\|\psi\|_{b, s}=\sup _{v \in V}\left(1+\sigma_{V}(v)\right)^{s}|\psi(v ; b)|, \quad \psi \in C^{\infty}(V), \quad b \in \mathscr{U}(\mathfrak{v}), \quad s \geq 0 .
$$

Then as usual, $\mathscr{C}\left(i v^{*}\right)$ and $\mathscr{C}(V)$ are isomorphic via the Fourier transforms:

$$
\begin{array}{ll}
\hat{\varphi}(v)=\int_{i \mathfrak{v}^{*}} e^{h}(v) \varphi(h) d h, & \varphi \in \mathscr{C}\left(i \mathfrak{v}^{*}\right), \quad v \in V \\
\hat{\psi}(h)=\int_{V} e^{-h}(v) \psi(v) d v, & \psi \in \mathscr{C}(V), h \in i v^{*} .
\end{array}
$$

For an appropriate normalization of $d h$, we have $\hat{\hat{\psi}}=\psi$.

Lemma 2.16. There is a family $\left\{\beta_{t}\right\}, t>1$, of functions in $C_{c}^{\infty}\left(i \mathfrak{v}^{*}\right)$ with the following property. For any $\varphi \in \mathscr{C}\left(i \mathrm{v}^{*}\right), t>1$, write $\varphi_{t}=\beta_{t} \cdot \varphi \in C_{c}^{\infty}\left(i \mathrm{v}^{*}\right)$. Then given $v \in \mathscr{U}(\mathfrak{v}), s \geq 0$, there is a continuous seminorm $\gamma$ on $\mathscr{C}(V)$ so that $\left\|\hat{\varphi}-\left(\varphi_{t}\right)^{\wedge}\right\|_{b, s} \leq \gamma(\hat{\varphi}) / t$ for all $\varphi \in \mathscr{C}\left(i \mathfrak{v}^{*}\right), t>1$.

Proof. See [8, Lemma 12.14].

Now for $f \in \mathscr{C}(G)$ and a fixed $x \in G$, define $\psi(v)=f(v x), v \in V$. Then $\psi \in \mathscr{C}(V)$ since $\|\psi\|_{b, s} \leq \mu(1,1, b, 0, s)(f) \Xi(x)$, so that $\varphi=\hat{\psi} \in \mathscr{C}\left(i \mathrm{v}^{*}\right)$. Define $\left\{\beta_{t}\right\}$ and $\varphi_{t}=\beta_{t} \cdot \varphi, t>1$, as in Lemma 2.16, and set

$$
\begin{gathered}
f_{t}(v: x)=\hat{\varphi}_{t}(v), \quad v \in V \\
f_{t}(x)=f_{t}(1: x) .
\end{gathered}
$$

Lemma 2.18. Let $\mu$ be a continuous seminorm on $\mathscr{C}(G)$. Then there exists a continuous seminorm $\mu^{\prime}$ so that $\mu\left(f-f_{t}\right) \leq \mu^{\prime}(f) / t$ for all $f \in \mathscr{C}(G), t>1$.

Proof. See [8, Lemma 12.15].

Lemma 2.19. Let $f \in \mathscr{C}(G)$. Then $f \in \mathscr{C}_{H}(G)$ if and only if $f_{\zeta} \in \mathscr{C}_{H}(G / Z, \zeta)$ for all $\zeta \in \hat{Z}$. 
Proof. Recall from [5, §6] that $f_{H}(x)=\int_{\widehat{Z}}\left(f_{\zeta}\right)_{H}(x) d \zeta$. Thus $\left(f_{H}\right)_{\zeta}=\left(f_{\zeta}\right)_{H}$ for all $\zeta, H$. But now $f \in \mathscr{C}_{H}(G)$ if and only if $f_{H^{\prime}}=0$ for all $H^{\prime} \neq H$ if and only if $\left(f_{H^{\prime}}\right)_{\zeta}=\left(f_{\zeta}\right)_{H^{\prime}}=0$ for all $H^{\prime} \neq H$, all $\zeta$, if and only if $f_{\zeta} \in \mathscr{C}_{H}(G / Z, \zeta)$ for all $\zeta$. Q.E.D.

Proof of Theorem 2.12. We know [6, 6.20] that $K_{1}$-finite functions are dense in $\mathscr{C}(G)$. We also have the compact group $V / Z$ acting on $\mathscr{C}(G)$ by the adjoint action. As in [6, 6.20], ad $(V / Z)$-finite functions in $\mathscr{C}(G)$ are dense. Thus to show $\mathscr{C}(G)_{K}$ is dense in $\mathscr{C}(G)$, it is enough to show that any $K_{1}$-finite, $\operatorname{ad}(V / Z)$-finite $f \in \mathscr{C}(G)$ can be approximated by functions in $\mathscr{C}(G)_{K}$. But if $f$ is a $K_{1}$ - and $\operatorname{ad}(V / Z)$-finite, so are all the $f_{t}, t>1$. Further, for all $h \in i \mathrm{v}^{*}$, using the notation of (2.17),

$$
\left(e^{h} *_{V} f_{t}\right)(x)=\int_{V} e^{-h}(v) f_{t}(v: x) d v=\varphi_{t}(h)=\beta_{t}(h) \varphi(h)=0
$$

unless $h \in \operatorname{supp} \beta_{t}$. Thus $f_{t} \in \mathscr{C}(G)_{K}$ for all $t>1$. But using Lemma 2.18, $f_{t} \rightarrow f$ in $\mathscr{C}(G)$ as $t \rightarrow \infty$. Thus $\mathscr{C}(G)_{K}$ is dense in $\mathscr{C}(G)$.

To prove that $\mathscr{C}_{H}(G) \cap \mathscr{E}(G)_{K}$ is dense in $\mathscr{C}_{H}(G)$, it suffices as in [8] to prove that $f_{t} \in \mathscr{C}_{H}(G)$ whenever $f \in \mathscr{C}_{H}(G)$. For $h \in i \mathfrak{v}^{*}$, let $\zeta(h)=\left.e^{h}\right|_{Z}$, and for $\zeta \in \hat{Z}$, let $\left(i v^{*}\right)_{\zeta}=\left\{h \in i v^{*}: \zeta(h)=\zeta\right\}$. This is a discrete subset of $i \mathfrak{v}^{*}$ since $V / Z$ is compact. Now for $f \in \mathscr{C}_{H}(G), f_{\zeta} \in \mathscr{C}_{H}(G / Z, \zeta)$ for all $\zeta \in \widehat{Z}$ by Lemma 2.19. Hence $e^{h}{ }_{V} f=e^{h}{ }_{V / Z} f_{\zeta(h)} \in \mathscr{C}_{H}(G / Z, \zeta(h))$ for all $h \in i v^{*}$. But $f_{t}(x)=\int_{i 0^{*}} \beta_{t}(h)\left(e^{h}{ }_{V} f\right)(x) d h$, so

$$
\left(f_{t}\right)_{\zeta}(x)=\sum_{h \in\left(i 0^{*}\right)_{\zeta}} \beta_{t}(h)\left(e^{h} *_{V} f\right)(x)
$$

is a finite (since $\beta_{t}$ has compact support) linear combination of functions in $\mathscr{C}_{H}(G / Z, \zeta)$, hence lies in $\mathscr{C}_{H}(G / Z, \zeta)$. Thus $f_{t} \in \mathscr{C}_{H}(G)$ using Lemma 2.19. Q.E.D.

\section{WAVE PACKETS CORRESPONDING TO SCHWARTZ FUNCTIONS}

For $f \in \mathscr{C}(G)$, the Plancherel theorem expands $f$ in terms of the distribution characters, $\Theta(H: \chi)$, of tempered representations of $G$ parametrized by Cartan subgroups $H$ of $G$ and characters $\chi \in \widehat{H}$. As in (1.1) we can write

$$
f(x)=\sum_{H \in \operatorname{Car}(G)} f_{H}(x),
$$

where

$$
f_{H}(x)=\int_{\widehat{H}} \Theta(H: \chi)(R(x) f) m(H: \chi) d \chi .
$$

Fix $H \in \operatorname{Car}(G)$. We may assume $H$ is $\theta$-stable and write $H=T \times A$, where $T=H \cap K, A=\exp (\mathfrak{a}), \mathfrak{a}=\mathfrak{h} \cap \mathfrak{p}$. We first will make more precise the contribution of $H$ to the Plancherel formula. 
Let $P=M A N$ be a parabolic subgroup associated to $H$. We recall the parametrization of the $H$-series of tempered representations from [9].

Let $\Phi_{M}=\Phi(\mathfrak{m}, \mathfrak{t})$ denote the roots of $\mathfrak{m}$ with respect to $\mathfrak{t}, \Phi_{M}^{+}$a choice of positive roots. Let $\rho_{M}$ denote the half-sum over $\Phi_{M}^{+}$and let $\Phi_{M, K}^{+}=$ $\Phi_{M}^{+} \cap \Phi\left(\mathfrak{k}_{M}, \mathfrak{t}\right)$. Then set

$$
\begin{aligned}
\Lambda_{M}=\left\{\lambda \in i t^{*}: \lambda-\rho_{M}\right. & \text { is integral, } \\
& \left.\lambda \text { is } \Phi_{M} \text {-nonsingular and } \Phi_{M, K}^{+} \text {-dominant }\right\} ;
\end{aligned}
$$

$$
\begin{aligned}
& \pi_{\lambda}^{0}: \text { the relative discrete series representation of } M^{0} \\
& \text { corresponding to } \lambda \in \Lambda_{M} ; \\
& X(\lambda)=\left\{\chi \in Z_{M^{M}}\left(M^{0}\right)^{\wedge}:\left.\chi\right|_{Z_{M^{0}}} \text { is a multiple of }\left.e^{\lambda-\rho_{M}}\right|_{Z_{M^{0}}}\right\} ; \\
& \pi(H: \chi: \lambda: \nu)=\operatorname{Ind}_{Z_{M} G\left(M^{0}\right) M^{0} A N}\left(\chi \otimes \pi_{\lambda}^{0} \otimes e^{i \nu} \otimes 1\right), \\
& \quad \lambda \in \Lambda_{M}, \quad \chi \in X(\lambda), \nu \in \mathscr{F}=\mathfrak{a}^{*} ;
\end{aligned}
$$

$\Theta(H: \chi: \lambda: \nu):$ the character of $\pi(H: \chi: \lambda: \nu)$.

Recall $K=K_{1} \times V$ from Proposition 2.1. Write $K_{M}^{0}=M^{0} \cap K$. Proposition 2.1 can also be applied to $K_{M}^{0}$ to write $K_{M}^{0}=K_{1, M}^{0} \times V_{M}$, where $K_{1, M}^{0}=$ $K_{1} \cap M^{0}$ is maximal compact in $K_{M}^{0}$. Note $V_{M} \not \subset V$ in general. We can now write $\Lambda_{M}$ as the union of continuous families defined as follows.

For $h \in i \mathfrak{v}^{*}=\left\{h \in i \mathfrak{k}^{*}: h\left(\mathfrak{k}_{1}\right)=0\right\}$, set $h_{M}(h)=\left.h\right|_{\mathfrak{t}}$. Let $\Phi_{M, 1}^{+}=\left\{\alpha \in \Phi_{M}^{+}\right.$: $\left\langle\alpha, h_{M}(h)\right\rangle=0$ for all $\left.h \in i \mathfrak{v}^{*}\right\}$ and

$$
\begin{aligned}
\Lambda_{M, 0}=\left\{\lambda_{0} \in i t^{*}:\right. & \lambda_{0}\left(\mathfrak{v}_{M}\right)=0, \lambda_{0}-\rho_{M} \text { is integral, and } \\
& \left.\lambda_{0} \text { is } \Phi_{M, K}^{+} \text {-dominant and } \Phi_{M, 1}^{+} \text {-nonsingular }\right\}
\end{aligned}
$$

For $\lambda_{0} \in \Lambda_{M, 0}$, let $\mathscr{C}\left(\lambda_{0}\right)$ denote the set of connected components of $\{h \in$ $i \mathfrak{v}^{*}:\left\langle\beta, \lambda_{0}+h_{M}(h)\right\rangle \neq 0$ for all $\left.\beta \in \Phi_{M}^{+} \backslash \Phi_{M, 1}^{+}\right\}$. Then $\Lambda_{M}$ is the disjoint union of continuous families

$$
\left\{\lambda_{0}+h_{M}(h): h \in \mathscr{D}\right\}, \quad \text { where } \lambda_{0} \in \Lambda_{M, 0} \text { and } \mathscr{D} \in \mathscr{C}\left(\lambda_{0}\right) .
$$

Let $X_{1}=\left\{h \in i v^{*}: h_{M}(h)=0\right\}$. Thus the families $\left\{\lambda_{0}+h_{M}(h): h \in i \mathfrak{v}^{*}\right\}$ are really parametrized by cosets in $i v^{*} / X_{1}$. Define an equivalence relation on $X\left(\lambda_{0}\right)$ by $\chi \sim \chi^{\prime}$ if $\chi^{\prime}=\chi \otimes e^{h}$ for some $h \in X_{1}$. (Note for $h \in X_{1}$, $\left.e^{h}\right|_{Z_{M^{0}}}=\left.e^{h_{M}(h)}\right|_{Z_{M^{0}}}=1$ so $\chi \otimes e^{h} \in X\left(\lambda_{0}\right)$ whenever $\chi \in X\left(\lambda_{0}\right), h \in X_{1}$.) Let $\left[X\left(\lambda_{0}\right) / X_{1}\right]$ denote a set of representatives for the equivalence classes of $X\left(\lambda_{0}\right)$ with respect to $\sim$. Then we can parametrize relative discrete series representations of $M^{\dagger}=Z_{M}\left(M^{0}\right) M^{0}$ as follows. 
Lemma 3.3. Let $\lambda \in \Lambda_{M}, \chi \in X(\lambda)$. Then there are $\lambda_{0} \in \Lambda_{M, 0}, \chi_{0} \in$ $\left[X\left(\lambda_{0}\right) / X_{1}\right]$, and $h \in i v^{*}$ such that $\lambda=\lambda_{0}+h_{M}(h)$ and $\chi=\chi_{0} \otimes e^{h}$. Further, for $\lambda_{0}, \lambda_{0}^{\prime} \in \Lambda_{M, 0}, \chi_{0} \in\left[X\left(\lambda_{0}\right) / X_{1}\right], \chi_{0}^{\prime} \in\left[X\left(\lambda_{0}^{\prime}\right) / X_{1}\right]$, and $h, h^{\prime} \in i v^{*}$, $\lambda_{0}+h_{M}(h)=\lambda_{0}^{\prime}+h_{M}\left(h^{\prime}\right)$ and $\chi_{0} \otimes e^{h}=\chi_{0}^{\prime} \otimes e^{h^{\prime}}$ if and only if $\lambda_{0}=\lambda_{0}^{\prime}, \chi_{0}=\chi_{0}^{\prime}$, and $h \equiv h^{\prime}(\bmod S)$, where $S=\left\{h \in i \mathrm{v}^{*}:\left.e^{h}\right|_{T}=1\right\}$.

Proof. Write $\mathfrak{t}=\mathfrak{t}_{1} \oplus \mathfrak{v}_{M}$, where $\mathfrak{t}_{1}=\mathfrak{t} \cap \mathfrak{k}_{1}$. For $\lambda \in \Lambda_{M}, \lambda_{0}=\left.\lambda\right|_{\mathfrak{t}_{1}} \in$ $\Lambda_{M, 0}$, and, as in [9, $\left.\S 3\right]$, there is $h_{0} \in i \mathfrak{v}^{*}$ such that $\left.\lambda\right|_{\mathfrak{v}_{M}}=h_{M}\left(h_{0}\right)$. Then $\lambda=\lambda_{0}+h_{M}\left(h_{0}\right)$. Now $\chi \otimes e^{-h_{0}} \in X\left(\lambda_{0}\right)$ and so there are $\chi_{0} \in\left[X\left(\lambda_{0}\right) / X_{1}\right]$ and $h_{1} \in X_{1}$ with $\chi \otimes e^{-h_{0}}=\chi_{0} \otimes e^{h_{1}}$, i.e., $\chi=\chi_{0} \otimes e^{h_{0}+h_{1}}$. Now if we take $h=h_{0}+h_{1}$ we have $\chi=\chi_{0} \otimes e^{h}$ and $\lambda=\lambda_{0}+h_{M}(h)$ since $h_{M}\left(h_{1}\right)=0$.

Then

Now assume $\lambda_{0}, \lambda_{0}^{\prime} \in \Lambda_{M, 0}$ and $h, h^{\prime} \in i v^{*}$ with $\lambda_{0}+h_{M}(h)=\lambda_{0}^{\prime}+h_{M}\left(h^{\prime}\right)$.

and

$$
\lambda_{0}=\lambda_{0}+\left.h_{M}(h)\right|_{\mathfrak{t}_{1}}=\lambda_{0}^{\prime}+\left.h_{M}\left(h^{\prime}\right)\right|_{\mathfrak{t}_{1}}=\lambda_{0}^{\prime}
$$

$$
h_{M}(h)=\lambda_{0}+\left.h_{M}(h)\right|_{\mathfrak{v}_{M}}=\lambda_{0}^{\prime}+\left.h_{M}\left(h^{\prime}\right)\right|_{\mathfrak{v}_{M}}=h_{M}\left(h^{\prime}\right) \text {. }
$$

Thus $h \equiv h^{\prime}\left(\bmod X_{1}\right)$. Now if $\chi_{0}, \chi_{0}^{\prime} \in\left[X\left(\lambda_{0}\right) / X_{1}\right]$ with $\chi_{0} \otimes e^{h}=\chi_{0}^{\prime} \otimes e^{h^{\prime}}$ we have $\chi_{0}^{\prime}=\chi_{0} \otimes e^{h-h^{\prime}}$, where $h-h^{\prime} \in X_{1}$ so that $\chi_{0}=\chi_{0}^{\prime}$. Thus $\chi_{0} \otimes$ $e^{h}=\chi_{0} \otimes e^{h^{\prime}}$ so $\left.e^{h-h^{\prime}}\right|_{Z_{M}\left(M^{0}\right)}=1$. But $S=\left\{h:\left.e^{h}\right|_{Z_{M}\left(M^{0}\right)}=1\right\} \cap X_{1}$, so $h \equiv h^{\prime}(\bmod S)$. Q.E.D.

We can now rewrite $(3.1 \mathrm{~b})$ more precisely as

$$
\begin{aligned}
f_{H}(x)= & c_{H} \sum_{\lambda_{0} \in \Lambda_{M} .0} \sum_{\chi_{0} \in\left[X\left(\lambda_{0}\right) / X_{1}\right]} \operatorname{deg} \chi_{0} \\
& \times \int_{i v^{*} / S} \int_{\mathscr{F}} \Theta\left(H: \chi_{0} \otimes e^{h}: \lambda_{0}+h_{M}(h): \nu: R(x) f\right) \\
& \times m\left(H: \chi_{0} \otimes e^{h}: \lambda_{0}+h_{M}(h): \nu\right) d h d \nu,
\end{aligned}
$$

where $c_{H}$ is a constant depending on normalizations of Haar measures, $m\left(H: \chi_{0} \otimes e^{h}: \lambda_{0}+h_{M}(h): \nu\right)$ is the Plancherel function defined in [5, 6.17], $d \nu$ is Lebesgue measure on $\mathscr{F}=\mathfrak{a}^{*}$, and $d \dot{h}$ is the quotient of Lebesgue measure on $i \mathrm{v}^{*} / S$. (Note $S$ is a discrete subgroup of $i \mathrm{v}^{*}$ since $K / T$ is compact.)

In order to write $f_{H}$ as a sum of wave packets which can be compared with those defined in [9], we need to expand $f$ in terms of $K$-types. In order for all sums to be finite, we need to assume that $f$ is $K$-compact.

Recall $Z=Z_{G} \cap V \subseteq Z_{M}\left(M^{0}\right)$. For $\chi \in Z_{M}\left(M^{0}\right)^{\wedge}$, let $\widehat{K}(\chi)=\{\tau \in \widehat{K}$ : $\tau(k z)=\zeta(z) \tau(k)$ for all $k \in K, z \in Z$, where $\zeta$ is the $Z$-character of $\chi\}$. Then all $K$-types of the representation $\pi\left(H: \chi_{0} \otimes e^{h}: \lambda_{0}+h_{M}(h): \nu\right)$ lie in $\widehat{K}\left(\chi_{0} \otimes e^{h}\right)=\left\{\tau_{h}=\tau_{0} \otimes e^{h}: \tau_{0} \in \widehat{K}\left(\chi_{0}\right)\right\}$. Since $Z \subseteq T$ we have $\left.e^{h}\right|_{Z}=1$ for all $h \in S$. Thus $\chi_{0} \otimes e^{h} \in \widehat{K}\left(\chi_{0}\right)$ for all $\chi_{0} \in \widehat{K}\left(\chi_{0}\right), h \in S$, and we can 
define an equivalence relation on $\widehat{K}\left(\chi_{0}\right)$ by $\chi \sim \chi^{\prime}$ if $\chi^{\prime}=\chi \otimes e^{h}, h \in S$. Let $\left[\widehat{K}\left(\chi_{0}\right) / S\right]$ denote a complete set of representatives for the corresponding equivalence classes.

Theorem 3.5. Suppose $f \in \mathscr{C}(G)_{K}$. Then

$$
\begin{aligned}
f_{H}(x)= & c_{H} \sum_{\lambda_{0} \in \Lambda_{M, 0}} \sum_{\mathscr{D} \in \mathscr{C}\left(\lambda_{0}\right)} \sum_{\chi_{0} \in\left[X\left(\lambda_{0}\right) / X_{1}\right]} \operatorname{deg} \chi_{0} \\
& \times \sum_{\tau_{1} \in \widehat{K}\left(\chi_{0}\right)} \sum_{\tau_{2} \in\left[\widehat{K}\left(\chi_{0}\right) / S\right]} f\left(H: \lambda_{0}: \mathscr{D}: \chi_{0}: \tau_{1}: \tau_{2}: x\right),
\end{aligned}
$$

where

(3.6a) $\hat{f}\left(H: \lambda_{0}: \chi_{0}: \tau_{1}: \tau_{2}: h: \nu: x\right)$

$$
=\delta\left(\tau_{1, h}^{*}\right) *_{K / Z} \Theta\left(H: \chi_{0} \otimes e^{h}: \lambda_{0}+h_{M}(h): \nu: R(x) f\right) *_{K / Z} \delta\left(\tau_{2, h}\right)
$$

and

$$
\begin{aligned}
f(H & \left.: \lambda_{0}: \mathscr{D}: \chi_{0}: \tau_{1}: \tau_{2}: x\right) \\
& =\int_{\mathscr{D} \times \mathscr{F}} \hat{f}\left(H: \lambda_{0}: \chi_{0}: \tau_{1}: \tau_{2}: h: \nu: x\right) m\left(\chi_{0} \otimes e^{h}: \lambda_{0}+h_{M}(h): \nu\right) d h d \nu .
\end{aligned}
$$

Further, only finitely many of the functions $f\left(H: \lambda_{0}: \mathscr{D}: \chi_{0}: \tau_{1}: \tau_{2}\right)$ are nonzero.

We will refer to $f\left(H: \lambda_{0}: \mathscr{D}: \chi_{0}: \tau_{1}: \tau_{2}\right)$ as a wave packet corresponding to $f$, so that for $f$ a $K$-compact Schwartz function, Theorem 3.5 expresses $f_{H}$, and hence $f$, as a finite sum of wave packets. The remainder of this section is devoted to the proof of Theorem 3.5.

Lemma 3.7. There is a central subgroup $T_{b}$ of $T$ so that $T=T_{1} \cdot T_{b}, T_{1}=$ $T \cap K_{1}$.

Proof. Decompose $\mathfrak{g}=\mathfrak{g}_{a} \oplus \mathfrak{g}_{b}$, where $\mathfrak{g}_{a}$ is the sum of all simple ideals of $\mathfrak{g}$ which are compact or not of hermitian type, and $\mathfrak{g}_{b}$ is the sum of the center of $\mathfrak{g}$ and the noncompact ideals of hermitian type. Then $G=G_{a} G_{b}$, where $G_{a}$ has finite center and all Cartan subgroups of $G_{b}$ are abelian (see [5, $\left.\S 1\right]$ ). Write $T=T_{a} \cdot T_{b}$, where $T_{a}=T \cap G_{a}, T_{b}=T \cap G_{b}$. Then $T_{a}$ is compact, hence is contained in $T_{1} . T_{b}$ is abelian, hence central in $T$. Q.E.D.

Lemma 3.8. $\left[X\left(\lambda_{0}\right) / X_{1}\right]$ is a finite set.

Proof. $X\left(\lambda_{0}\right)$ is in bijective correspondence with $\widehat{T}\left(\lambda_{0}\right)=\left\{\eta \in \widehat{T}:\left.\eta\right|_{T^{0}}\right.$ is a multiple of $\left.e^{\lambda_{0}-\rho_{M}}\right\}$ by $\chi \leftrightarrow \chi \otimes e^{\lambda_{0}-\rho_{M}}=\eta$ on $T=Z_{M}\left(M^{0}\right) T^{0}$. Define an equivalence relation $\sim$ on $\widehat{T}\left(\lambda_{0}\right)$ by $\eta \sim \eta^{\prime}$ if $\eta^{\prime}=\eta \otimes e^{h}, h \in X_{1}$. Then the bijection preserves $\sim$ so that $\left[X\left(\lambda_{0}\right) / X_{1}\right]$ is in bijective correspondence with $\left[\widehat{T}\left(\lambda_{0}\right) / \sim\right]$, the set of equivalence classes in $\widehat{T}\left(\lambda_{0}\right)$. Thus it suffices to show that $\left[\widehat{T}\left(\lambda_{0}\right) / \sim\right]$ is finite. 
Recall from Lemma 3.7 that $T=T_{1} \cdot T_{b}$, where $T_{1}=T \cap K_{1}$ and $T_{b}$ is central in $T$. Thus $\varphi(\eta)=\left.\eta\right|_{T_{1}}$ defines a mapping from $\widehat{T}\left(\lambda_{0}\right)$ into $\widehat{T}_{1}\left(\lambda_{0}\right)=\left\{\eta_{1} \in\right.$ $\widehat{T}_{1}:\left.\eta_{1}\right|_{T_{1} \cap T^{0}}$ is a multiple of $\left.\left.e^{\lambda_{0}-\rho_{M}}\right|_{T_{1} \cap T^{0}}\right\}$. If $\eta \sim \eta^{\prime}$ we have $\varphi(\eta)=\varphi\left(\eta^{\prime}\right)$ since $\left.e^{h}\right|_{T_{1}}=1$ for all $h \in i \mathrm{v}^{*}$. Thus $\varphi$ factors through $\left[\widehat{T}\left(\lambda_{0}\right) / \sim\right]$. Suppose $\varphi(\eta)=\varphi\left(\eta^{\prime}\right)$. Let $\zeta$ and $\zeta^{\prime}$ denote the $T_{b}$-characters of $\eta$ and $\eta^{\prime}$. Then $\zeta^{\prime} \zeta^{-1}$ is a character of $T_{b} / T_{b} \cap T_{1}$, hence the restriction of $e^{h}$ for some $h \in i \mathrm{v}^{*}$. Thus $\zeta=\zeta^{\prime} \otimes e^{h}$ and so $\eta=\eta^{\prime} \otimes e^{h}$. But $\left.\eta\right|_{T^{0}}=\left.\eta^{\prime}\right|_{T^{0}}$ so $\left.e^{h}\right|_{T^{0}}=1$ and $h \in X_{1}$, i.e., $\eta \sim \eta^{\prime}$. Thus $\varphi:\left[\widehat{T}\left(\lambda_{0}\right) / \sim\right] \rightarrow \widehat{T}_{1}\left(\lambda_{0}\right)$ is injective. But $T_{1}$ is compact so that $T_{1} / T_{1} \cap T^{0}$ is finite. Thus $\widehat{T}_{1}\left(\lambda_{0}\right)$ and hence $\left[\widehat{T}\left(\lambda_{0}\right) / \sim\right]$ are finite. Q.E.D.

Lemma 3.9. Suppose $f \in \mathscr{C}(G)_{K}$. Then there is a compact subset $\omega$ of $i t^{*}$ so that $\Theta(H: \chi: \lambda: \nu)(R(x) f)=0$ for all $x \in G, \chi \in X(\lambda), \nu \in \mathscr{F}$, unless $\lambda \in \omega \cap \Lambda_{M}$.

Proof. Let $\mathfrak{b}$ be a Cartan subalgebra of $\mathfrak{k}$ containing $\mathfrak{t}$. For $\tau \in \widehat{K}$, set $\|\tau\|=$ $\|\mu\|$, where $\mu$ is the highest weight of $\tau$ and $\|\cdot\|$ is the norm on $i b^{*}$ coming from the Killing form. Now if $f \in \mathscr{C}(G)_{K}$ there is a $c \geq 0$ so that $\delta\left(\tau^{*}\right){ }_{K} f=$ 0 unless $\tau \in \Omega_{c}=\{\tau \in \widehat{K}:\|\tau\| \leq c\}$. For $\lambda \in \Lambda_{M}$, the corresponding relative discrete series representation $\pi_{\lambda}^{0}$ of $M^{0}$ has minimal $K_{M}^{0}$-type $\lambda-\rho(\mathscr{C})$, where $\rho(\mathscr{C})$ is a shift depending only on the chamber $\mathscr{C}$ of $\lambda$. Thus all $K_{M}^{0}$-types $\sigma$ of $\pi_{\lambda}^{0}$ satisfy

$$
\|\sigma\| \geq c(\lambda)=\|\lambda-\rho(\mathscr{C})\| .
$$

Now if $\tau$ is a $K$-type of $\operatorname{Ind}_{M^{\dagger} A N}^{G}\left(\chi \otimes \pi_{\lambda}^{0} \otimes e^{i \nu} \otimes 1\right),\left.\tau\right|_{K_{M}^{0}}$ contains some $K_{M}^{0}$-type $\sigma$ of $\pi_{\lambda}^{0}$. Let $\mu_{\sigma}$ be a weight of $\tau$ such that $\left.\mu_{\sigma}\right|_{\mathfrak{t}}$ is the highest weight of $\sigma$. Then $\|\tau\|=\|\mu\| \geq\left\|\mu_{\sigma}\right\| \geq\left\|\left.\mu_{\sigma}\right|_{t}\right\|=\|\sigma\| \geq c(\lambda)$. Thus if $c(\lambda)>c$, we have $\tau \notin \Omega_{c}$ for all $K$-types $\tau$ of the induced representation. Thus $\Theta(H: \chi: \lambda: \nu)(R(x) f)=0$ unless $\lambda \in \Lambda_{M} \cap \omega_{c}$, where $\omega_{c}=\left\{\lambda \in i t^{*}:\right.$ $\left.\|\lambda\| \leq c+\max _{\mathscr{C}}\|\rho(\mathscr{C})\|\right\}$. Q.E.D.

Let $f \in \mathscr{C}(G)_{K}$ and pick $c$ such that all $K$-types of $f$ are in $\Omega_{c}=\{\tau \in \widehat{K}$ : $\|\tau\| \leq c\}$. Define $\omega_{c}$ as in the proof of Lemma 3.9 so that

$$
\Theta(H: \chi: \lambda: \nu)(R(x) f)=0
$$

unless $\lambda \in \Lambda_{M} \cap \omega_{c}$. Let $F_{0}=\left\{\lambda_{0} \in \Lambda_{M, 0}: \lambda_{0}+h_{M}(h) \in \omega_{c}\right.$ for some $\left.h \in i v^{*}\right\}$.

Lemma 3.10. $F_{0}$ is finite and there is $m \geq 0$ so that $\omega_{c} \cap \Lambda_{M} \subset\left\{\lambda_{0}+h_{M}(h)\right.$ : $\lambda_{0} \in F_{0}$ and $\left.\left\|h_{M}(h)\right\| \leq m\right\}$.

Proof. Although $\mathfrak{t}_{1}$ and $\mathfrak{v}_{M}$ are not orthogonal with respect to the Killing form, $\mathfrak{t}=\mathfrak{t}_{1} \oplus \mathfrak{v}_{M}$ is a direct sum so there is $c_{1}>0$ so that $\left(\left\|\lambda_{0}\right\|^{2}+\left\|h_{M}(h)\right\|^{2}\right)^{1 / 2} \leq$ $c_{1}\left\|\lambda_{0}+h_{M}(h)\right\|$ for all $\lambda_{0} \in \Lambda_{M, 0}, h \in i v^{*}$. Thus, if $\lambda_{0}+h_{M}(h) \in \omega_{c}$, $\left(\left\|\lambda_{0}\right\|^{2}+\left\|h_{M}(h)\right\|^{2}\right)^{1 / 2} \leq c_{1} c^{\prime}$, where $c^{\prime}=c+\max _{\mathscr{E}}\|\rho(\mathscr{C})\|$. Thus, if $m=c_{1} c^{\prime}$ 
we have $\left\|\lambda_{0}\right\| \leq m$ and $\left\|h_{M}(h)\right\| \leq m$. But $\Lambda_{M, 0}$ is discrete so that $F_{0} \subseteq$ $\left\{\lambda_{0} \in \Lambda_{M, 0}:\left\|\lambda_{0}\right\| \leq m\right\}$ is finite. Q.E.D.

Lemma 3.11. $\left\{h \in \mathrm{iv}^{*} / S:\left\|h_{M}(h)\right\| \leq m\right\}$ is compact in $i \mathfrak{v}^{*} / S$.

Proof. It suffices to prove that $X_{1} / S$ is compact, where $X_{1}=\left\{h \in i \mathrm{v}^{*}\right.$ : $\left.h_{M}(h)=0\right\}=\left\{h \in i v^{*}:\left.e^{h}\right|_{T^{0}}=1\right\}$. But $\left.h \rightarrow e^{h}\right|_{T}$ defines a homeomorphism between $X_{1} / S$ and $\left(T / T_{1} \cdot T^{0}\right)^{\wedge}$. But $T / T_{1} \cdot T^{0}$ is discrete so that $\left(T / T_{1} \cdot T^{0}\right)^{\wedge}$ is compact. Q.E.D.

Proof of Theorem 3.5. Let $f \in \mathscr{C}(G)_{K}$ with $K$-types in the compact set $\Omega_{c}$. Pick $m$ and $F_{0}$ as in Lemma 3.10 so that

$$
\Theta\left(H: \chi_{0} \otimes e^{h}: \lambda_{0}+h_{M}(h): \nu: R(x) f\right)=0
$$

unless $\lambda_{0} \in F_{0}$ and $h \in i v^{*} / S$ with $\left\|h_{M}(h)\right\| \leq m$. By Lemma 3.11 there are $m^{\prime} \geq 0$ and a fundamental domain $\mathscr{V}_{m^{\prime}}$ for $i v^{*} / S$ so that $\|h\| \leq m^{\prime}$ for all $h \in \mathscr{V}_{m^{\prime}}$. Thus we can rewrite (3.4) as

$$
f_{H}(x)=c_{H} \sum_{\lambda_{0} \in F_{0}} \sum_{\chi_{0} \in\left[X\left(\lambda_{0}\right) / X_{1}\right]} \operatorname{deg} \chi_{0} f_{H}\left(\chi_{0}: \lambda_{0}: x\right),
$$

where

$$
\begin{aligned}
f_{H}\left(\chi_{0}: \lambda_{0}: x\right)=\int_{\mathscr{V}_{m^{\prime}}} \int_{\mathscr{F}} \Theta\left(H: \chi_{0} \otimes e^{h}: \lambda_{0}+h_{M}(h): \nu: R(x) f\right) \\
\times m\left(H: \chi_{0} \otimes e^{h}: \lambda_{0}+h_{M}(h): \nu\right) d h d \nu .
\end{aligned}
$$

Fix $\lambda_{0} \in F_{0}$ and $\chi_{0} \in\left[X\left(\lambda_{0}\right) / X_{1}\right]$ and write

$$
\Theta(h: \nu)=\Theta\left(H: \chi_{0} \otimes e^{h}: \lambda_{0}+h_{M}(h): \nu\right)
$$

and $m(h: \nu)=m\left(H: \chi_{0} \otimes e^{h}: \lambda_{0}+h_{M}(h): \nu\right)$. For each $h \in \mathscr{V}_{m^{\prime}}$ we can write (3.13) $\Theta(h: \nu)(R(x) f)=\sum_{\tau_{1}, \tau_{2} \in \widehat{K}\left(\chi_{0}\right)} \delta\left(\tau_{1, h}^{*}\right) *_{K / Z} \Theta(h: \nu: R(x) f) *_{K / Z} \delta\left(\tau_{2, h}\right)$.

But $\delta\left(\tau_{1, h}^{*}\right){ }_{K / Z} \Theta(h: \nu: R(x) f){ }_{K / Z} \delta\left(\tau_{2, h}\right)=0$ unless $\tau_{1, h}, \tau_{2, h} \in \Omega_{c}$, i.e., unless $\tau_{1}, \tau_{2} \in\left\{\tau \in \widehat{K}\left(\chi_{0}\right):\|\tau\| \leq c+m^{\prime}\right\}$. Since $\widehat{K}\left(\chi_{0}\right)$ is discrete, this is a finite set, so the double sum in (3.13) has only finitely many nonzero terms. Thus we have

$$
\begin{aligned}
f_{H}\left(\chi_{0}: \lambda_{0}: x\right)= & \int_{i 0^{*} / S} \int_{\mathscr{F}}\left[\sum_{\tau_{1}, \tau_{2} \in \widehat{K}\left(\chi_{0}\right)} \delta\left(\tau_{1, h}^{*}\right) * \Theta(h: \nu: R(x) f) * \delta\left(\tau_{2, h}\right)\right] \\
& \times m(h: \nu) d h d \nu \\
& =\sum_{\tau \in \widehat{K}\left(\chi_{0}\right)} \sum_{\tau_{2} \in\left[\widehat{K}\left(\chi_{0}\right) / S\right]} \int_{i v^{*} \times \mathscr{F}} \hat{f}\left(H: \lambda_{0}: \chi_{0}: \tau_{1}: \tau_{2}: h: \nu: x\right)
\end{aligned}
$$


Finally, we can write $i \mathfrak{v}^{*}=\bigcup_{\mathscr{D} \in \mathscr{C}\left(\lambda_{0}\right)} \mathscr{D}$ to obtain the formula in Theorem 3.5. Note $\mathscr{C}\left(\lambda_{0}\right)$ and $\left[X\left(\lambda_{0}\right) / X_{1}\right]$ are finite sets, and $f\left(H: \lambda_{0}: \mathscr{D}: \chi_{0}: \tau_{1}: \tau_{2}\right)=0$ unless $\lambda_{0} \in F_{0}$ and $\tau_{1}, \tau_{2} \in\left\{\tau \in \widehat{K}\left(\chi_{0}\right):\|\tau\| \leq c+m^{\prime}\right\}$. Q.E.D.

\section{EISENSTEIN INTEgRALS ASSOCIATED to SCHWARTZ FUNCTIONS}

Throughout this and the next four sections we will fix a $\theta$-stable Cartan subgroup $H$ of $G$, parameters $\lambda_{0} \in \Lambda_{M, 0}$ and $\chi_{0} \in X\left(\lambda_{0}\right)$, and a chamber $\mathscr{D} \in \mathscr{C}\left(\lambda_{0}\right)$ so that

$$
\left\{\Theta(h: \nu)=\Theta\left(H: \chi_{0} \otimes e^{h}: \lambda_{0}+h_{M}(h): \nu\right): h \in \mathscr{D}, \nu \in \mathscr{F}\right\}
$$

is a family of characters of $H$-series representations as in (3.2). Fix $\tau_{1}, \tau_{2} \in$ $\widehat{K}\left(\chi_{0}\right)$. Then for any $f \in \mathscr{C}(G)$ we can define

$$
\hat{f}(h: \nu: x)=\delta\left(\tau_{1, h}^{*}\right) *_{K / Z} \Theta(h: \nu)(R(x) f) *_{K / Z} \delta\left(\tau_{2, h}\right)
$$

as in (3.6a). In this notation,

$$
f\left(H: \lambda_{0}: \mathscr{D}: \chi_{0}: \tau_{1}: \tau_{2}\right)=\int_{\mathscr{D} \times \mathscr{F}} \hat{f}(h: \nu: x) m(h: \nu) d h d \nu
$$

is a wave packet associated to $f$. In Theorem 3.5 we showed that if $f$ is $K$-compact it is a finite sum of wave packets. However, the definition (4.1) of wave packets associated to $f$ can be made for any $f \in \mathscr{C}(G)$. The main result of this section is Theorem 4.6, which shows how to write $\hat{f}(h: \nu)$ as an Eisenstein integral. This will allow us to relate the wave packets corresponding to $f \in \mathscr{C}(G)$ to the wave packets of Eisenstein integrals constructed in [9].

Let $\tau_{j}^{1}$ denote the restriction of $\tau_{j}$ to $K_{1}, j=1,2$, and let $W=E_{\tau_{1}^{1}} \otimes E_{\tau_{2}^{1}}$ be the finite-dimensional subspace of $L^{2}\left(K_{1} \times K_{1}\right)$ on which $K_{1}$ acts on the left and right by representations ' $\tau_{1}^{1}$ and ' $\tau_{2}^{1}$ equivalent to multiples of $\tau_{1}^{1}$ and $\tau_{2}^{1}$ respectively. Then for each $h \in \mathfrak{v}_{\mathbf{C}}^{*}, K=K_{1} \times V$ acts on $W$ on the left and right as in $[9,6.4 \mathrm{c}]$ by representations $\left({ }^{\prime} \tau_{1, h},{ }^{\prime} \tau_{2, h}\right)$, where ${ }^{\prime} \tau_{i, h}={ }^{\prime} \tau_{i}^{1} \otimes e^{h_{i}+h}$, $i=1,2$. Here $e^{h_{i}}$ is the character by which $\tau_{i}$ acts on $V$. Thus if we define

$$
\begin{gathered}
f(h: x)=\delta\left(\tau_{1, h}^{*}\right) *_{K} f(x) *_{K / Z} \delta\left(\tau_{2, h}\right), \quad f \in \mathscr{C}(G), \\
F(h: x)\left(k_{1}: k_{2}\right)=f\left(h: k_{1}^{-1} x k_{2}^{-1}\right), \quad k_{1}, k_{2} \in K_{1},
\end{gathered}
$$

$F(h: x) \in W$ is a $\tau$-spherical function, i.e.,

$$
F\left(h: k_{1} x k_{2}\right)={ }^{\prime} \tau_{1, h}\left(k_{1}\right) F(h: x)^{\prime} \tau_{2, h}\left(k_{2}\right), \quad k_{1}, k_{2} \in K .
$$

For $m \in M^{\dagger}, \nu \in \mathscr{F}$, define

$$
G_{f}(h: \nu: m)=\int_{A N} F(h: m a n) e^{-\left(i \nu-\rho_{p}\right)(\log a)} d a d n,
$$


where $\rho_{p}(H)$ is $\frac{1}{2}$ the trace of $\operatorname{ad}(H)$ on $\mathfrak{n}, H \in \mathfrak{a}$. As in $[6,5.3]$, we have $G_{f}(h: \nu) \in \mathscr{C}\left(M^{\dagger} / Z, \zeta(h): W\right)$, where $\zeta(h)=\left.e^{h_{1}+h}\right|_{Z}=\left.e^{h_{2}+h}\right|_{Z}$, and it follows from $(4.2 \mathrm{c})$ that

$$
\begin{aligned}
G_{f}\left(h: \nu: k_{1} m k_{2}\right)={ }^{\prime} \tau_{1, h}\left(k_{1}\right) G_{f}(h: \nu: m)^{\prime} \tau_{2, h}\left(k_{2}\right), & \\
& k_{1}, k_{2} \in K_{M}^{\dagger}=K \cap M^{\dagger} .
\end{aligned}
$$

Finally for all $h \in \operatorname{cl}(\mathscr{D}), \nu \in \mathscr{F}, m \in M^{\dagger}$ we define

$$
\begin{aligned}
\Psi_{f}(h: \nu: m) & =\left[M / M^{\dagger}\right] \Theta\left(M^{\dagger}: h: R(m) G_{f}(h: \nu)\right) \\
& =\left[M / M^{\dagger}\right] \int_{M^{\dagger} / Z} \overline{\Theta\left(M^{\dagger}: h: y\right)} G_{f}(h: \nu: y m) d(y Z),
\end{aligned}
$$

where, for $h \in \mathscr{D}, \Theta\left(M^{\dagger}: h\right)$ denotes the character of the relative discrete series representation $\pi\left(M^{\dagger}: h\right)$ of $M^{\dagger}$ corresponding to $\chi_{0} \otimes e^{h} \in Z_{M}\left(M^{0}\right)^{\text {- }}$ and $\lambda_{0}+h_{M}(h) \in i \mathrm{t}^{*}$. For $h \in \operatorname{cl}(\mathscr{D}) \backslash \mathscr{D}, \Theta\left(M^{\dagger}: h\right)$ denotes the character of the limit of discrete series coming from $\mathscr{D}$. Then $\Psi_{f}(h: \nu)$ is a spherical function of matrix coefficients for $\pi\left(M^{\dagger}: h\right)$ with $K_{M}^{\dagger}$-types $\left.{ }^{\prime} \tau_{1, h}\right|_{K_{M}^{\dagger}}$ and $\left.{ }^{\prime} \tau_{2, h}\right|_{K_{M}^{\dagger}}$.

Extend $\Psi_{f}(h: \nu)$ to $G$ by

$$
\Psi_{f}(h: \nu: k m a n)={ }^{\prime} \tau_{1, h}(k) \Psi_{f}(h: \nu: m) \text { for } k \operatorname{man} \in K M^{\dagger} A N,
$$

and define the Eisenstein integral as

$$
E\left(P: \Psi_{f}(h: \nu): h: \nu: x\right)=\int_{K / Z} \Psi_{f}(h: \nu: x k)^{\prime} \tau_{2, h}\left(k^{-1}\right) e^{\left(i \nu-\rho_{p}\right) H_{p}(x k)} d(k Z) \text {. }
$$

Theorem 4.6. $E\left(P: \Psi_{f}(h: \nu): h: \nu: x\right)(1: 1)=\hat{f}(h: \nu: x)$ for all $f \in \mathscr{C}(G)$, $h \in \operatorname{cl}(\mathscr{D}), \nu \in \mathfrak{a}^{*}$, and $x \in G$.

\section{Lemma 4.7.}

$$
\begin{aligned}
& \Theta(h:: \nu)(R(x) F(h)) \\
&= \Theta(h: \nu)\left(L\left(x^{-1}\right) F(h)\right) \\
&=\left[M / M^{\dagger}\right] \int_{K / Z} \int_{M^{\dagger} / Z} \int_{A N} \overline{\Theta\left(M^{\dagger}: h: m\right)} F\left(h: x k m a n k^{-1}\right) \\
& \times e^{-\left(i \nu-\rho_{p}\right)(\log a)} d a d n d m d k .
\end{aligned}
$$

Proof. The standard result $[6,5.10]$ is

$$
\begin{aligned}
\Theta & (h: \nu)\left(L\left(x^{-1}\right) F(h)\right) \\
& =\int_{K / Z} \int_{M / Z} \int_{A N} \overline{\Theta(M: h: m)} F\left(h: x k \operatorname{mank}^{-1}\right) e^{-\left(i \nu-\rho_{p}\right)(\log a)} d a d n d m d k,
\end{aligned}
$$

where $\Theta(M: h)=\operatorname{Ind}_{M^{\dagger}}^{M}\left(\theta\left(M^{\dagger}: h\right)\right)$. But $\Theta(M: h)$ is supported on $M^{\dagger}$ and is given there by $\boldsymbol{\Theta}(M: h: m)=\sum_{y \in M / M^{\dagger}} \Theta\left(M^{\dagger}: h: y m y^{-1}\right), m \in M^{\dagger}$. 
Since we can assume $y \in M \cap K$, the result now follows by changing variables in the integral: $m \rightarrow y^{-1} m y, k \rightarrow k y, n \rightarrow y^{-1} n y$. Q.E.D.

Proof of Theorem 4.6. Write $x k=k_{0} m_{0} a_{0} n_{0} \in K M^{\dagger} A N$. Now

$$
\begin{array}{rl}
\int_{A N} & F\left(h: x k m a n k^{-1}\right) e^{-\left(i \nu-\rho_{p}\right)(\log a)} d a d n \\
= & { }^{\prime} \tau_{1, h}\left(k_{0}\right) \int_{A N} F\left(h: m_{0} m a_{0} a(m a)^{-1} n_{0}(m a) n\right) \\
& \times e^{-\left(i \nu-\rho_{p}\right)(\log a)} d a d n^{\prime} \tau_{2, h}\left(k^{-1}\right) \\
= & { }^{\prime} \tau_{1, h}\left(k_{0}\right) e^{\left(i \nu-\rho_{p}\right)\left(\log a_{0}\right)} \int_{A N} F\left(h: m_{0} m a n\right) e^{-\left(i \nu-\rho_{p}\right)(\log a)} d a d n^{\prime} \tau_{2, h}\left(k^{-1}\right)
\end{array}
$$

by changing variables $n \rightarrow\left[(m a)^{-1} n_{0} m a\right]^{-1} n$ and $a \rightarrow a_{0}^{-1} a$. But this last expression equals $e^{\left(i \nu-\rho_{p}\right)\left(\log a_{0}\right){ }^{\prime}} \tau_{1, h}\left(k_{0}\right) G_{f}\left(h: \nu: m_{0} m\right)^{\prime} \tau_{2, h}\left(k^{-1}\right)$. Thus

$$
\begin{aligned}
& {\left[M / M^{\dagger}\right] \int_{M^{\dagger} / Z} \int_{A N} \overline{\Theta\left(M^{\dagger}: h: m\right)} F\left(h: x k m a n k^{-1}\right) e^{-\left(i \nu-\rho_{p}\right)(\log a)} d a d n d m} \\
& =e^{\left(i \nu-\rho_{p}\right)\left(\log a_{0}\right){ }^{\prime}} \tau_{1, h}\left(k_{0}\right) \Psi_{f}\left(h: \nu: m_{0}\right)^{\prime} \tau_{2, h}\left(k^{-1}\right) \\
& =e^{\left(i \nu-\rho_{p}\right)(H(x k))} \Psi_{f}(h: \nu: x k)^{\prime} \tau_{2, h}\left(k^{-1}\right) \text { as } x k=k_{0} m_{0} a_{0} n_{0} .
\end{aligned}
$$

Thus, using Lemma 4.7,

$$
\Theta(h: \nu)(R(x) F(h))=E\left(P: \Psi_{f}(h: \nu): h: \nu: x\right) .
$$

But $\hat{f}(h: \nu: x)=\Theta(h: \nu)(R(x) F(h))(1: 1)$. Q.E.D.

Note that we have proved a spherical function version of Theorem 4.6. Namely, if we define

$$
\widehat{F}(h: \nu: x)=\Theta(h: \nu)(R(x) F(h)),
$$

we have

$$
\widehat{F}(h: \nu: x)=E\left(P: \Psi_{f}(h: \nu): h: \nu: x\right)
$$

\section{SPHERICAL FUNCTIONS OF MATRIX COEFFICIENTS}

Let $\lambda_{0} \in \Lambda_{M, 0}, \chi_{0} \in X\left(\lambda_{0}\right)$, and $\mathscr{D} \in \mathscr{D}\left(\lambda_{0}\right)$ as in $\S 4$ so that $\left\{\pi\left(M^{\dagger}: h\right)=\right.$ $\left.\chi(h) \otimes \pi_{\lambda_{0}+h_{M}(h)}^{0}: h \in \mathscr{D}\right\}$ is a family of relative discrete series representations of $M^{\dagger}$ as in $\S 3$. Fix $\tau_{1}, \tau_{2} \in \widehat{K}\left(\chi_{0}\right)$ and define $W=E_{\tau_{1}^{1}} \otimes E_{\tau_{2}^{1}} \subseteq L^{2}\left(K_{1} \times K_{1}\right)$ as in $\S 4$. For each $h \in \mathfrak{v}_{\mathrm{C}}^{*}, K$ acts on the right and left of $W$ by representations $\left({ }^{\prime} \tau_{1, h},{ }^{\prime} \tau_{2, h}\right)$. For $j=1,2, h \in \operatorname{cl}(\mathscr{D})$, define

(5.1a) $\Sigma_{j, h}=\left\{\sigma \in \widehat{K}_{M}^{\dagger}: \sigma\right.$ occurs in both $\left.\tau_{j} \otimes e^{h}\right|_{K_{M}^{\dagger}}$ and in $\left.\left.\pi\left(M^{\dagger}: h\right)\right|_{K_{M}^{\dagger}}\right\}$. 
By [9, 4.9], there is $\Sigma_{j} \subseteq K_{M}^{\dagger}$ so that $\Sigma_{j, h}=\left\{\sigma_{h}=\sigma \otimes e^{h}: \sigma \in \Sigma_{j}\right\}$ for all $h \in \operatorname{cl}(\mathscr{D})$. For $h \in \operatorname{cl}(\mathscr{D})$ define

(5.1b) $\mathscr{H}\left(M^{\dagger}: \sigma_{j}: h\right):$ the $\sigma_{j, h}$-isotypic subspace of the representation

$$
\text { space of } \pi\left(M^{\dagger}: h\right)
$$

$\mathscr{V}\left(M^{\dagger}: \sigma_{1}: \sigma_{2}: h\right)$ : the linear span in $C^{\infty}\left(M^{\dagger}\right)$ of the matrix coefficients

$$
\begin{aligned}
& x \mapsto\left\langle\pi\left(M^{\dagger}: h\right)(x) w_{2}, w_{1}\right\rangle, w_{j} \in \mathscr{H}\left(M^{\dagger}: \sigma_{j}: h\right) ; \\
& \mathscr{V}\left(M^{\dagger}: \tau_{1}: \tau_{2}: h\right)=\bigoplus \sum_{\left(\sigma_{1}, \sigma_{2}\right) \in \Sigma_{1} \times \Sigma_{2}} \mathscr{V}\left(M^{\dagger}: \sigma_{1}: \sigma_{2}: h\right)
\end{aligned}
$$

$\mathscr{S}\left(M^{\dagger}: W: h\right):$ the vector space of all $F: M^{\dagger} \rightarrow W$ such that

(i) $F\left(k_{1} m k_{2}\right)={ }^{\prime} \tau_{1, h}\left(k_{1}\right) F(m)^{\prime} \tau_{2, h}\left(k_{2}\right)$

for all $m \in M^{\dagger}, k_{1}, k_{2} \in K_{M}^{\dagger}$;

(ii) $m \rightarrow\left\langle F(m), w^{*}\right\rangle$ is an element of $\mathscr{V}\left(M^{\dagger}: \tau_{1}: \tau_{2}: h\right)$

for all $w^{*} \in W^{*}$.

Elements of $\mathscr{S}\left(M^{\dagger}: W: h\right)$ are $K_{M}^{\dagger}$-spherical functions of matrix coefficients of $\pi\left(M^{\dagger}: h\right)$. For each $f \in \mathscr{C}(G)$, the functions $m \rightarrow \Psi_{f}(h: \nu: m)$ defined in (4.4) satisfy $\Psi_{f}(h: \nu) \in \mathscr{S}\left(M^{\dagger}: W: h\right)$ for all $h \in \operatorname{cl}(\mathscr{D}), \nu \in \mathscr{F}$.

We define holomorphic families of $K_{M}^{\dagger}$-spherical functions as follows.

$\mathscr{S}\left(M^{\dagger}: W\right)$ : the set of all $F \in C^{\infty}\left(\mathfrak{v}_{\mathrm{C}}{ }^{*} \times M^{\dagger}: W\right)$ satisfying

(i) $F(h) \in \mathscr{S}\left(M^{\dagger}: W: h\right)$ for all $h \in \operatorname{cl}(\mathscr{D})$;

(ii) $h \mapsto F(h: m)$ is a holomorphic $W$-valued function on $\mathfrak{v}_{\mathbf{C}}^{*}$ for all $m \in M^{\dagger}$;

(iii) Let $\mathscr{D}_{\mathbf{C}}$ be a complex neighborhood of $\mathscr{D}$ in ${ }^{{ }^{*}}{ }_{\mathrm{C}}$ of the form $\mathscr{D}_{\mathrm{C}}=\mathscr{D}+i \omega$, where $\omega \subseteq i v^{*}$ is a neighborhood of 0 with compact closure. Then for all $D_{1}, D_{2} \in \mathscr{U}(\mathfrak{m})$ there are $C, r \geq 0$ so that $\left\|F\left(h: D_{1} ; x ; D_{2}\right)\right\| \leq C(1+|h|)^{r}\left(1+d(h)^{-1}\right)^{r} e^{\left|h_{I}\right| \sigma_{V}(x)} \Xi_{M}(x)$ for all $h \in \mathscr{D}_{\mathbf{C}}, x \in M^{\dagger}$. Here $d(h)$ denotes the distance from $h_{R}$ to the boundary of $\mathscr{D}$ if $h=h_{R}+i h_{I} \in \mathscr{D}+i \omega$.

In this section we will show how to find consistent bases for the vector spaces $\mathscr{S}\left(M^{\dagger}: W: h\right)$ given in terms of the holomorphic families of $K_{M}^{\dagger}$-spherical 
functions in $\mathscr{S}\left(M^{\dagger}: W\right)$. Given $f \in \mathscr{C}(G)$, this will allow us to find families $F_{i} \in \mathscr{S}\left(M^{\dagger}: W\right)$ and $\alpha_{i}: \operatorname{cl}(\mathscr{D}) \times \mathscr{F} \rightarrow \mathbf{C}$ so that

$$
\Psi_{f}(h: \nu)=\sum_{i} \alpha_{i}(h: \nu) F_{i}(h) .
$$

Using Theorem 4.6 this will allow us to write

$$
\int_{\mathscr{D} \times \mathscr{F}} \hat{f}(h: \nu: x) m(h: \nu) d h d \nu=\sum_{i} \Phi_{i}(x)(1: 1),
$$

where

$$
\Phi_{i}(x)=\int_{\mathscr{D} \times \mathscr{F}} E\left(P: F_{i}: h: \nu: x\right) \alpha_{i}(h: \nu) m(h: \nu) d h d \nu
$$

is a wave packet of Eisenstein integrals of the type studied in [9].

We will also construct bases for the dual spaces $\mathscr{S}\left(M^{\dagger}: W: h\right)^{*}$. These dual bases, together with information on the properties of $\Psi_{f}$ as a function on $\mathscr{D} \times \mathscr{F} \times M^{\dagger}$ which will be established in $\S \S 6$ and 7 , will allow us in $\S 8$ to study the properties of the coefficients $\alpha_{i}$ as functions of $h$ and $\nu$. The main result on consistent bases is Theorem 5.32. For technical reasons, it is stated for $M^{\prime}$, the universal cover of $M^{0}$, rather than $M^{\dagger}$.

In $[9, \S 4]$ we defined for each $\left(\sigma_{1}, \sigma_{2}\right) \in \Sigma_{1} \times \Sigma_{2}$ a subspace

$$
\mathscr{F}\left(M^{\dagger}: \sigma_{1}: \sigma_{2}\right) \subseteq C^{\infty}\left(\mathfrak{v}_{\mathrm{C}}^{*} \times M^{\dagger}\right)
$$

consisting of holomorphic families of relative discrete series matrix coefficients. In particular, each $\varphi \in \mathscr{F}\left(M^{\dagger}: \sigma_{1}: \sigma_{2}\right)$ satisfies

$$
\begin{gathered}
h \rightarrow \varphi(h: m) \text { is holomorphic on } \mathfrak{v}_{\mathrm{C}}^{*} \text { for all } m \in M^{\dagger} ; \\
\varphi(h) \in \mathscr{V}\left(M^{\dagger}: \sigma_{1}: \sigma_{2}: h\right) \quad \text { for all } h \in \operatorname{cl}(\mathscr{D}) .
\end{gathered}
$$

Corresponding to each $\varphi \in \mathscr{F}\left(M^{\dagger}: \sigma_{1}: \sigma_{2}\right)$ we defined in [9, §5] a holomorphic family $F(\varphi) \in \mathscr{S}\left(M^{\dagger}: W\right)$. Define $K_{M, 1}^{V}=K_{M}^{\dagger} \cdot V \cap K_{1}$ and let $W^{\prime \prime}$ be the kernel in $W$ of restriction of functions to $K_{M, 1}^{V} \times K_{M, 1}^{V}$. Let $W^{\prime}$ be the orthocomplement of $W^{\prime \prime}$. Then $F(\varphi)$ is characterized uniquely by

$$
F(\varphi: h: m)\left(k_{1}: k_{2}\right)=\zeta\left(\tau_{1}: h: v_{1}^{-1}\right) \zeta\left(\tau_{2}: h: v_{2}^{-1}\right) \varphi\left(h: k_{1, M}^{-1} m k_{2, M}^{-1}\right),
$$

where $k_{i}=v_{i} k_{i, M} \in\left(V \cdot K_{M}^{\dagger}\right) \cap K_{M, 1}^{V}$ and $\zeta\left(\tau_{i}: h\right)$ denotes the $V$-character of $\tau_{i, h}=\tau_{i} \otimes e^{h}$.

The families $F(\varphi), \varphi \in \mathscr{F}\left(M^{\dagger}: \sigma_{1}: \sigma_{2}\right)$, were the basic holomorphic families of $K_{M}^{\dagger}$-spherical functions used to define Schwartz wave packets of Eisenstein integrals in [9]. However, the only properties of the families used 
in the proofs were properties (i)-(iii) of the definition (5.2) of $\mathscr{S}\left(M^{\dagger}: W\right)$. In order to obtain consistent bases for the families $\mathscr{S}\left(M^{\dagger}: W: h\right)$, we need to expand the construction slightly, keeping within the family $\mathscr{S}\left(M^{\dagger}: W\right)$, by going to the universal covering group of $M^{0}$.

Recall the family $\left\{\pi\left(M^{\dagger}: h\right): h \in \mathscr{D}\right\}$ used to define $\mathscr{S}\left(M^{\dagger}: W: h\right)$ is of the form $\pi\left(M^{\dagger}: h\right)=\chi(h) \otimes \pi\left(M^{0}: h_{M}(h)\right)$, where $\chi(h)=e^{h} \otimes \chi_{0} \in Z_{M}\left(M^{0}\right)^{\wedge}$ and $\left\{\pi\left(M^{0}: h_{M}(h)\right)=\pi_{\lambda_{0}+h_{M}(h)}^{0}: h \in \mathscr{D}\right\}$ is a family of relative discrete series representations of $M^{0}$. Write $\mathscr{D}_{M}=\left\{h_{M}(h): h \in \mathscr{D}\right\}$. Define

(5.6a) $\Sigma_{j}^{0}=\left\{\sigma^{0} \in \widehat{K}_{M^{0}}: \sigma_{h}^{0}=\sigma^{0} \otimes e^{h}\right.$ occurs in both $\left.\tau_{j, h}\right|_{K_{M}^{0}}$ and in $\left.\left.\pi\left(M^{0}: h_{M}(h)\right)\right|_{K_{M}^{0}}\right\}$.

It is independent of $h \in \mathrm{cl}(\mathscr{D})$, and for all $h \in \operatorname{cl}\left(\mathscr{D}_{M}\right)$, we define

$$
\mathscr{V}\left(M^{0}: \tau_{1}: \tau_{2}: h\right)=\bigoplus \sum_{\left(\sigma_{1}^{0}, \sigma_{2}^{0}\right) \in \Sigma_{1}^{0} \times \Sigma_{2}^{0}} \mathscr{V}\left(M^{0}: \sigma_{1}^{0}: \sigma_{2}^{0}: h\right),
$$

where $\mathscr{V}\left(M^{0}: \sigma_{1}^{0}: \sigma_{2}^{0}: h\right)$ denotes the space of matrix coefficients of $\pi\left(M^{0}: h\right)$ corresponding as in (5.1c) to the $K_{M^{0}}$-type $\left(\sigma_{1, h}^{0}, \sigma_{2, h}^{0}\right)$.

Further, since $\left.\pi\left(M^{\dagger}: h\right)\right|_{K_{M}^{\dagger}}=\left.\chi(h) \otimes \pi\left(M^{0}: h\right)\right|_{K_{M}^{0}}$, it is clear that $\Sigma_{j}=$ $\left\{\chi(0) \otimes \sigma^{0}: \sigma^{0} \in \Sigma_{j}^{0}\right\}, j=1,2$. Define

$$
\mathscr{F}\left(M^{0}: \tau_{1}: \tau_{2}\right)=\bigoplus \sum_{\left(\sigma_{1}^{0}, \sigma_{2}^{0}\right) \in \Sigma_{1}^{0} \times \Sigma_{2}^{0}} \mathscr{F}\left(M^{0}: \sigma_{1}^{0}: \sigma_{2}^{0}\right),
$$

where $\mathscr{F}\left(M^{0}: \sigma_{1}^{0}: \sigma_{2}^{0}\right)$ denotes the space of holomorphic families of matrix coefficients for the $\pi\left(M^{0}: h\right), h \in \mathscr{D}_{M}$, corresponding to the $K_{M^{0}}^{0}$-types $\left(\sigma_{1, h}^{0}, \sigma_{2, h}^{0}\right)$ as in $[9,4.7]$. Then each element of $\mathscr{V}\left(M^{\dagger}: \tau_{1}: \tau_{2}: h\right)$ is a finite sum of terms of the form $\varphi_{h}\left(z m^{0}\right)=\psi_{h}(z) \varphi_{h}^{0}\left(m^{0}\right), z \in Z_{M}\left(M^{0}\right), m^{0} \in M^{0}$, where $\psi_{h}$ is a matrix coefficient of $\chi(h)$ and $\varphi_{h}^{0} \in \mathscr{V}\left(M^{0}: \tau_{1}: \tau_{2}: h_{M}(h)\right)$. Further, each element of $\mathscr{F}\left(M^{\dagger}: \tau_{1}: \tau_{2}\right)$ is a finite sum of terms of the form $\varphi\left(h: z m^{0}\right)=\psi(h: z) \varphi^{0}\left(h_{M}(h): m^{0}\right)$, where $\psi(0)$ is a matrix coefficient of $\chi(0), \psi(h: z)=e^{h}(z) \psi(0: z)$, and $\varphi^{0} \in \mathscr{F}\left(M^{0}: \tau_{1}: \tau_{2}\right)$.

Let $p: M^{\prime} \rightarrow M^{0}$ denote the universal covering group. Then $K_{M^{\prime}}=$ $\left[K_{M^{\prime}}, K_{M^{\prime}}\right] \times V_{M^{\prime}}$, where $\left[K_{M^{\prime}}, K_{M^{\prime}}\right]$ is compact and $V_{M^{\prime}}$ is a vector group. In the notation of $[9,4.7], \mathfrak{v}_{M^{\prime}}=\mathfrak{v}_{M} \oplus \mathfrak{u}_{M}$ and $\mathscr{D}_{M} \subseteq i \mathfrak{v}_{M}^{*}$ is just $\left\{h \in \mathscr{D}_{M^{\prime}}\right.$ : $\left.h\left(\mathfrak{u}_{M}\right)=0\right\}$, where $\mathscr{D}_{M^{\prime}} \subseteq i \mathfrak{v}_{M^{\prime}}^{*}$ is the connected component of $\left\{h \in i \mathfrak{v}_{M^{\prime}}^{*}\right.$ : $\left\langle\beta, \lambda_{0}+h\right\rangle \neq 0$ for $\left.\beta \in \Phi_{M}^{\dagger} \backslash \Phi_{M, K}^{\dagger}\right\}$ that contains $\mathscr{D}_{M}$.

For $h \in \mathscr{D}_{M^{\prime}}$, let $\pi\left(M^{\prime}: h\right)$ denote the relative discrete series representation corresponding to $\lambda_{0}+h$. Then for all $h \in \mathscr{D}_{M}$,

$$
\pi\left(M^{\prime}: h\right)=\pi\left(M^{0}: h\right) \circ p .
$$


Further, for each $\left(\sigma_{1}^{0}, \sigma_{2}^{0}\right) \in \Sigma_{1}^{0} \times \Sigma_{2}^{0}$, there are $\left(\sigma_{1}^{\prime}, \sigma_{2}^{\prime}\right) \in \widehat{K}_{M^{\prime}}$ so that $\sigma_{i, h}^{\prime}=$ $\left.\sigma_{i, h}^{0} \circ p\right|_{K_{M^{\prime}}}$ for all $h \in\left(\mathfrak{v}_{M}\right)_{\mathbf{C}}^{*}$. Let $\Sigma_{i}^{\prime}=\left\{\sigma_{i}^{\prime}: \sigma_{i}^{0} \in \Sigma_{i}^{0}\right\}$. For each $\left(\sigma_{1}^{\prime}, \sigma_{2}^{\prime}\right) \in$ $\Sigma_{1}^{\prime} \times \Sigma_{2}^{\prime}, h \in \mathscr{D}_{M^{\prime}}$, define $\mathscr{V}\left(M^{\prime}: \sigma_{1}^{\prime}: \sigma_{2}^{\prime}: h\right)$ to be the space of matrix coefficients of $\pi\left(M^{\prime}: h\right)$ corresponding to $\left(\sigma_{1, h}^{\prime}, \sigma_{2, h}^{\prime}\right)$, and set

$$
\mathscr{V}\left(M^{\prime}: \tau_{1}: \tau_{2}: h\right)=\bigoplus \sum_{\left(\sigma_{1}^{\prime}, \sigma_{2}^{\prime}\right) \in \Sigma_{1}^{\prime} \times \Sigma_{2}^{\prime}} \mathscr{V}\left(M^{\prime}: \sigma_{1}^{\prime}: \sigma_{2}^{\prime}: h\right) .
$$

We also define

$$
\mathscr{F}\left(M^{\prime}: \tau_{1}: \tau_{2}\right)=\bigoplus \sum \mathscr{F}\left(M^{\prime}: \sigma_{1}^{\prime}: \sigma_{2}^{\prime}\right),
$$

where $\mathscr{F}\left(M^{\prime}: \sigma_{1}^{\prime}: \sigma_{2}^{\prime}\right)$ is the space of holomorphic families of matrix coefficients for $\pi\left(M^{\prime}: h\right), h \in \mathscr{D}_{M^{\prime}}$, defined as in $[9,4.6]$ corresponding to $\left(\sigma_{1, h}^{\prime}, \sigma_{2, h}^{\prime}\right)$. Then if $h \in \mathscr{D}_{M}$, for each $\varphi_{h}^{0} \in \mathscr{V}\left(M^{0}: \tau_{1}: \tau_{2}: h\right)$ there is $\varphi_{h}^{\prime} \in \mathscr{V}\left(M^{\prime}: \tau_{1}: \tau_{2}: h\right)$ with $\varphi_{h}^{\prime}=\varphi_{h} \circ p$. Further, each $\varphi^{0} \in \mathscr{F}\left(M^{0}: \tau_{1}: \tau_{2}\right)$ is obtained from a family $\varphi^{\prime} \in \mathscr{F}\left(M^{\prime}: \tau_{1}: \tau_{2}\right)$ so that $\varphi^{\prime}(h)=\varphi^{0}(h) \circ p$ whenever $h \in \mathscr{D}_{M}$.

For each $h \in \operatorname{cl}(\mathscr{D}), Z_{M}\left(M^{0}\right)$ acts on $W$ by $\left.\left({ }^{\prime} \tau_{1, h},{ }^{\prime} \tau_{2, h}\right)\right|_{Z_{M}\left(M^{0}\right)}$. Let $W_{\chi}$ be the $(\chi(h), \chi(h))$-isotypic subspace for this action, and let

$$
W_{0}=\left\{w \in W_{\chi}:{ }^{\prime} \tau_{1, h}(z) w=w^{\prime} \tau_{2, h}(z) \text { for all } z \in Z_{M}\left(M^{0}\right)\right\} .
$$

$W_{0}$ is independent of $h$, and is a $K_{M}^{0}$-invariant subspace of $W$ for

$$
\left.\left({ }^{\prime} \tau_{1, h},{ }^{\prime} \tau_{2, h}\right)\right|_{K_{M}^{0}}
$$

since $Z_{M}\left(M^{0}\right)$ centralizes $K_{M}^{0}$. For $h \in \operatorname{cl}\left(\mathscr{D}_{M}\right)$, define

$\mathscr{S}\left(M^{0}: W_{0}: h\right):$ the vector space of all $F^{0}: M^{0} \rightarrow W_{0}$ such that

(i) $F^{0}\left(k_{1} m k_{2}\right)={ }^{\prime} \tau_{1, h}\left(k_{1}\right) F^{0}(m)^{\prime} \tau_{2, h}\left(k_{2}\right)$ for all $m \in M^{0}$, $k_{1}, k_{2} \in K_{M}^{0}$

(ii) $m \rightarrow\left\langle F^{0}(m), w^{*}\right\rangle$ is an element of $\mathscr{V}\left(M^{0}: \tau_{1}: \tau_{2}: h\right)$ for all $w^{*} \in W_{0}^{*}$.

For all $h \in \operatorname{cl}\left(\mathscr{D}_{M^{\prime}}\right)$, define

$\mathscr{S}\left(M^{\prime}: W_{0}: h\right):$ the vector space of all $F^{\prime}: M^{\prime} \rightarrow W_{0}$ such that

(i) $F^{\prime}\left(k_{1} m k_{2}\right)=e^{h}\left(k_{1} k_{2}\right)^{\prime} \tau_{1,0}\left(p\left(k_{1}\right)\right) F^{\prime}(m)^{\prime} \tau_{2,0}\left(p\left(k_{2}\right)\right)$

for all $m \in M^{\prime}, k_{1}, k_{2} \in K_{M^{\prime}}$;

(ii) $m \rightarrow\left\langle F^{\prime}(m), w^{*}\right\rangle$ is an element of $\mathscr{V}\left(M^{\prime}: \tau_{1}: \tau_{2}: h\right)$

for all $w^{*} \in W_{0}^{*}$. 
Lemma 5.9. For all $h \in \operatorname{cl}(\mathscr{D}), \quad F \rightarrow F^{0}=\left.F\right|_{M^{0}}$ gives a vector space isomorphism between $\mathscr{S}\left(M^{\dagger}: W: h\right)$ and $\mathscr{S}\left(M^{0}: W_{0}: h_{M}(h)\right)$.

Proof. Every $K_{M}^{\dagger}$-type of $\pi\left(M^{\dagger}: h\right)$ is of the form $\chi(h) \otimes \sigma_{h_{M}(h)}^{0}$, where $\sigma_{h_{M}(h)}^{0}$ is a $K_{M}^{0}$-type of $\pi\left(M^{0}: h_{M}(h)\right)$. Thus every $F \in \mathscr{S}\left(M^{\dagger}: W: h\right)$ takes values in $W_{\chi}$. But, for all $z \in Z_{M}\left(M^{0}\right)$ and $m \in M^{0}$,

$$
{ }^{\prime} \tau_{1, h}(z) F\left(m^{0}\right)=F\left(z m^{0}\right)=F\left(m^{0} z\right)=F\left(m^{0}\right)^{\prime} \tau_{2, h}(z) .
$$

Thus $F^{0}=\left.F\right|_{M^{0}}$ takes values in $W_{0}$. Further, $\mathscr{V}\left(M^{\dagger}: \tau_{1}: \tau_{2}: h\right)$ is the set of all finite linear combinations of the products of matrix coefficients of $\chi(h)$ and elements of $\mathscr{V}\left(M^{0}: \tau_{1}: \tau_{2}: h_{M}(h)\right)$. Thus it is clear that $\left.F\right|_{M^{0}} \in$ $\mathscr{S}\left(M^{0}: W_{0}: h_{M}(h)\right)$. Also, if $\left.F\right|_{M^{0}} \equiv 0$, then $F\left(z m^{0}\right)={ }^{\prime} \tau_{1, h}(z) F\left(m^{0}\right)=0$ for all $z \in Z_{M}\left(M^{0}\right), m^{0} \in M^{0}$, so that $F \equiv 0$. Thus $\left.F \rightarrow F\right|_{M^{0}}$ is one-toone. But given $F^{0} \in \mathscr{S}\left(M^{0}: W_{0}: h_{M}(h)\right)$, we can define $F: M^{\dagger} \rightarrow W$ by $F\left(z m^{0}\right)={ }^{\prime} \tau_{1, h}(z) F\left(m^{0}\right)$. For $z_{i} k_{i}^{0} \in K_{M}^{\dagger}$,

$$
\begin{aligned}
F\left(z_{1} k_{1}^{0} z m^{0} z_{2} k_{2}^{0}\right) & =F\left(z_{1} z z_{2} k_{1}^{0} m^{0} k_{2}^{0}\right)={ }^{\prime} \tau_{1, h}\left(z_{1} z z_{2}\right) F^{0}\left(k_{1}^{0} m^{0} k_{2}^{0}\right) \\
& ={ }^{\prime} \tau_{1, h}\left(z_{1}\right)^{\prime} \tau_{1, h}(z)^{\prime} \tau_{1, h}\left(z_{2}\right)^{\prime} \tau_{1, h}\left(k_{1}^{0}\right) F^{0}\left(m^{0}\right)^{\prime} \tau_{2, h}\left(k_{2}^{0}\right) \\
& ={ }^{\prime} \tau_{1, h}\left(z_{1} k_{1}^{0}\right) F\left(z m^{0}\right)^{\prime} \tau_{2, h}\left(z_{2} k_{2}^{0}\right)
\end{aligned}
$$

since $Z_{M}\left(M^{0}\right)$ centralizes $K_{M}^{0}$ and $F^{0}\left(m^{0}\right) \in W_{0}$. Thus $F$ satisfies (i) of (5.1e). To check (ii) we identify $W^{*}$ with $W$ via the hermitian inner product for which $\left({ }^{\prime} \tau_{1, h},{ }^{\prime} \tau_{2, h}\right)$ is unitary. Then for all $w \in W$,

$$
\left\langle F\left(z m^{0}\right), w\right\rangle=\left\langle\tau_{1, h}(z) F^{0}\left(m^{0}\right), w\right\rangle=\left\langle F^{0}\left(m^{0}\right),{ }^{\prime} \tau_{1, h}(z)^{-1} w\right\rangle .
$$

Let $\left\{w_{1}, \ldots, w_{n}\right\}$ be an orthonormal basis for $W$ so that $w_{1}, \ldots, w_{t} \in W_{0}$ and $w_{t+1}, \ldots, w_{n} \in W_{0}^{\perp}$. Then

$$
\begin{aligned}
\left\langle F^{0}\left(m^{0}\right),{ }^{\prime} \tau_{1, h}(z)^{-1} w\right\rangle & =\sum_{i=1}^{n} \overline{\left\langle^{\prime} \tau_{1, h}(z)^{-1} w, w_{i}\right\rangle}\left\langle F^{0}\left(m^{0}\right), w_{i}\right\rangle \\
& =\sum_{i=1}^{t}\left\langle\tau_{1, h}(z) w_{i}, w\right\rangle\left\langle F^{0}\left(m^{0}\right), w_{i}\right\rangle
\end{aligned}
$$

since $F^{0}\left(m^{0}\right) \in W_{0}$. But for all $1 \leq i \leq t, z \rightarrow\left\langle{ }^{\prime} \tau_{1, h}(z) w_{i}, w\right\rangle$ is a matrix coefficient of $\chi(h)$ since $W_{0} \subseteq W_{\chi}$, and $m^{0} \rightarrow\left\langle F\left(m^{0}\right), w_{i}\right\rangle$ is an element of $\mathscr{V}\left(M^{0}: \tau_{1}: \tau_{2}: h_{M}(h)\right)$. Thus $z m^{0} \mapsto\left\langle F\left(z m^{0}\right), w\right\rangle$ is an element of $\mathscr{V}\left(M^{\dagger}: \tau_{1}: \tau_{2}: h\right)$. Thus $F \in \mathscr{S}\left(M^{\dagger}: \tau_{1}: \tau_{2}: h\right)$ with $\left.F\right|_{M^{0}}=F^{0}$. Q.E.D.

Lemma 5.10. For all $h \in \mathrm{cl}\left(\mathscr{D}_{M}\right), F^{0} \rightarrow F^{0} \circ p$ gives an isomorphism between the vector spaces $\mathscr{S}\left(M^{0}: W_{0}: h\right)$ and $\mathscr{S}\left(M^{\prime}: W_{0}: h\right)$. 
Proof. This is clear since

$$
\mathscr{V}\left(M^{\prime}: \tau_{1}: \tau_{2}: h\right)=\left\{\varphi_{h}^{\prime}=\varphi_{h} \circ p: \varphi_{h} \in \mathscr{V}\left(M^{0}: \tau_{1}: \tau_{2}: h\right)\right\}
$$

when $h \in \mathscr{D}_{M^{\prime}}$ and ' $\tau_{i, h}\left(p\left(k^{\prime}\right)\right)=e^{h}\left(k^{\prime}\right)^{\prime} \tau_{i, 0}\left(p\left(k^{\prime}\right)\right)$ for $h \in\left(\mathfrak{v}_{M}\right)_{\mathrm{C}}^{*}$. Q.E.D.

Combining Lemmas 5.9 and 5.10 we have for each $h \in \operatorname{cl}(\mathscr{D})$ an isomorphism $F \rightarrow F^{\prime}=\left.F\right|_{M^{0}} \circ p$ from $\mathscr{S}\left(M^{\dagger}: W: h\right)$ onto $\mathscr{S}\left(M^{\prime}: W_{0}: h\right)$. This allows us to work in $M^{\prime}$ rather than $M$.

Next we want to characterize the images of the families $F(\varphi), \varphi \in$ $\mathscr{F}\left(M^{\dagger}: \tau_{1}: \tau_{2}\right)$, and extend them from $h \in \operatorname{cl}\left(\mathscr{D}_{M}\right)$ to all $h \in\left(\mathfrak{v}_{M^{\prime}}\right)_{\mathbf{C}}^{*}$. Suppose $\varphi \in \mathscr{F}\left(M^{\dagger}: \tau_{1}: \tau_{2}\right)$ is of the form $\varphi\left(h: z m^{0}\right)=\psi(h: z) \varphi^{0}\left(h_{M}(h): m^{0}\right)$, where $\psi(h: z)=e^{h}(z) \psi(0: z)$ is a family of matrix coefficients of $\chi(h)$, and $\varphi^{0} \in \mathscr{F}\left(M^{0}: \tau_{1}: \tau_{2}\right)$. Pick $\varphi^{\prime} \in \mathscr{F}\left(M^{\prime}: \tau_{1}: \tau_{2}\right)$ so that $\varphi^{\prime}(h)=\varphi^{0}(h) \circ p$ for all $h \in \mathscr{D}_{M}$.

Lemma 5.11. For all $m^{\prime} \in M^{\prime}$ and $h \in \mathfrak{v}_{\mathbf{C}}^{*}, F\left(\varphi: h: p\left(m^{\prime}\right)\right) \in W_{0} \cap W^{\prime}$, and for $k_{1}, k_{2} \in K_{M, 1}^{V}$,

$$
\begin{aligned}
F\left(\varphi: h: p\left(m^{\prime}\right)\right)\left(k_{1}: k_{2}\right)= & \zeta\left(\tau_{1}: 0: v_{1}^{-1}\right) \zeta\left(\tau_{2}: 0: v_{2}^{-1}\right) \psi\left(0: z_{1}^{-1} z_{2}^{-1}\right) \\
& \times e^{h_{M}(h)}\left(k_{1}^{\prime} k_{2}^{\prime}\right) \varphi^{\prime}\left(h_{M}(h): k_{1}^{\prime-1} m^{\prime} k_{2}^{\prime-1}\right) .
\end{aligned}
$$

Here $k_{i}=v_{i} z_{i} p\left(k_{i}^{\prime}\right) \in V \cdot Z_{M}\left(M^{0}\right) \cdot K_{M}^{0} \cap K_{M, 1}^{V}$, where $k_{i}^{\prime} \in K_{M^{\prime}}$.

Proof. The first statement is obvious from (5.5a). Now use (5.5b) to write

$$
\begin{aligned}
& F\left(\varphi: h: p\left(m^{\prime}\right)\right)\left(k_{1}: k_{2}\right) \\
&=\zeta\left(\tau_{1}: h: v_{1}^{-1}\right) \zeta\left(\tau_{2}: h: v_{2}^{-1}\right) \varphi\left(h: z_{1}^{-1} p\left(k_{1}^{\prime}\right)^{-1} p\left(m^{\prime}\right) p\left(k_{2}^{\prime}\right)^{-1} z_{2}^{-1}\right) \\
&= \zeta\left(\tau_{1}: h: v_{1}^{-1}\right) \zeta\left(\tau_{2}: h: v_{2}^{-1}\right) \psi\left(h: z_{1}^{-1} z_{2}^{-1}\right) \varphi^{0}\left(h_{M}(h): p\left(k_{1}^{\prime-1} m^{\prime} k_{2}^{\prime-1}\right)\right) \\
&= e^{h}\left(v_{1}^{-1} v_{2}^{-1} z_{1}^{-1} z_{2}^{-1}\right) \zeta\left(\tau_{1}: 0: v_{1}^{-1}\right) \zeta\left(\tau_{2}: 0: v_{2}^{-1}\right) \\
& \times \psi\left(0: z_{1}^{-1} z_{2}^{-1}\right) \varphi^{\prime}\left(h_{M}(h): k_{1}^{\prime-1} m^{\prime} k_{2}^{\prime-1}\right) .
\end{aligned}
$$

Now $k_{i}=v_{i} z_{i} p\left(k_{i}^{\prime}\right) \in K_{1}$, so $e^{h}\left(k_{i}\right)=1$. Thus $e^{h}\left(v_{i}^{-1} z_{i}^{-1}\right)=e^{h}\left(p\left(k_{1}^{\prime}\right)\right)=$ $e^{h_{M}(h)}\left(k_{i}^{\prime}\right)$. Q.E.D.

For each $\varphi^{\prime} \in \mathscr{F}\left(M^{\prime}: \tau_{1}: \tau_{2}\right)$ and matrix coefficient $\psi(0)$ of $\chi(0)$ we now define $F\left(\varphi^{\prime}: \psi(0)\right):\left(\mathfrak{v}_{M^{\prime}}\right)_{\mathrm{C}}^{*} \times M^{\prime} \rightarrow W_{0} \cap W^{\prime}$ by

$$
\begin{aligned}
& F\left(\varphi^{\prime}: \psi(0): h: m\right)\left(k_{1}: k_{2}\right) \\
& \quad=\zeta\left(\tau_{1}: 0: v_{1}^{-1}\right) \zeta\left(\tau_{2}: 0: v_{2}^{-1}\right) \psi\left(0: z_{1}^{-1} z_{2}^{-1}\right) e^{h}\left(k_{1}^{\prime} k_{2}^{\prime}\right) \varphi^{\prime}\left(h: k_{1}^{\prime-1} m k_{2}^{\prime-1}\right),
\end{aligned}
$$

where $k_{i}=v_{i} z_{i} p\left(k_{i}^{\prime}\right) \in V \cdot Z_{M}\left(M^{0}\right) \cdot p\left(K_{M^{\prime}}\right) \cap K_{M, 1}^{V}$. 
Lemma 5.13. $F\left(\varphi^{\prime}: \psi(0)\right) \in C^{\infty}\left(\left(\mathfrak{v}_{M^{\prime}}\right)_{\mathrm{C}}^{*} \times M^{\prime}\right)$. For all $m \in M^{\prime}, h \rightarrow$ $F\left(\varphi^{\prime}: \psi(0): h: m\right)$ is holomorphic as a function of $h \in\left(\mathfrak{v}_{M^{\prime}}\right)_{\mathbf{C}}$. For each $h \in$ $\operatorname{cl}\left(\mathscr{D}_{M^{\prime}}\right), F\left(\varphi^{\prime}: \psi(0): h\right) \in \mathscr{S}\left(M^{\prime}: W_{0}: h\right)$. For $h \in \operatorname{cl}(\mathscr{D}),\left.F(\varphi: h)\right|_{M^{0}} \circ p=$ $F\left(\varphi^{\prime}: \psi(0): h_{m}(h)\right)$, where $\varphi$ is related to $\varphi^{\prime}$ and $\psi(0)$ as in (5.11).

Proof. The joint smoothness and holomorphicity are obvious since $\varphi^{\prime}$ is jointly smooth and $h \rightarrow \varphi^{\prime}(h: m)$ is holomorphic for all $m \in M^{\prime}$. Write $F\left(\varphi^{\prime}: \psi(0)\right)=F^{\prime}$ and let $b_{1}, b_{2} \in K_{M^{\prime}}$. Then for all $m \in M^{\prime}$ and $k_{1}, k_{2} \in$ $K_{M, 1}^{V}$,

$$
\begin{aligned}
& \left.e^{h}\left(b_{1} b_{2}\right){ }^{\prime} \tau_{1,0}\left(p\left(b_{1}\right)\right) F^{\prime}(h: m)^{\prime} \tau_{2,0}\left(p\left(b_{2}\right)\right)\right]\left(k_{1}: k_{2}\right) \\
& \quad=e^{h}\left(b_{1} b_{2}\right) \zeta\left(\tau_{1}: 0: u_{1}\right) \zeta\left(\tau_{2}: 0: u_{2}\right) F^{\prime}(h: m)\left(g_{1}^{-1} k_{1}: k_{2} g_{2}^{-1}\right),
\end{aligned}
$$

where $p\left(b_{i}\right)=u_{i} g_{i} \in V \cdot K_{M, 1}^{V}$. Now if we write $k_{i}=v_{i} z_{i} p\left(k_{i}^{\prime}\right)$ as in (5.12), we have $g_{1}^{-1} k_{1}=\left(u_{1} v_{1}\right) z_{1} p\left(b_{1}^{-1} k_{1}^{\prime}\right)$ and $k_{2} g_{2}^{-1}=\left(u_{2} v_{2}\right) z_{2} p\left(k_{2}^{\prime} b_{2}^{-1}\right)$, so using (5.12),

$$
\begin{aligned}
\left.e^{h}\left(b_{1} b_{2}\right) \Gamma^{\prime} \tau_{1,0}\left(p\left(b_{1}\right)\right) F^{\prime}(h: m)^{\prime} \tau_{2,0}\left(p\left(b_{2}\right)\right)\right]\left(k_{1}: k_{2}\right) \\
=e^{h}\left(b_{1} b_{2}\right) \zeta\left(\tau_{1}: 0: u_{1}\right) \zeta\left(\tau_{2}: 0: u_{2}\right) \zeta\left(\tau_{1}: 0: v_{1}^{-1} u_{1}^{-1}\right) \zeta\left(\tau_{2}: 0: v_{2}^{-1} u_{2}^{-1}\right) \\
\quad \times \psi\left(0: z_{1}^{-1} z_{2}^{-1}\right) e^{h}\left(b_{1}^{-1} k_{1}^{\prime} k_{2}^{\prime} b_{2}^{-1}\right) \varphi^{\prime}\left(h: k_{1}^{\prime-1} b_{1} m b_{2} k_{2}^{\prime-1}\right) \\
=F^{\prime}\left(h: b_{1} m b_{2}\right)\left(k_{1}: k_{2}\right) .
\end{aligned}
$$

Thus $F$ satisfies property (i) of $(5.8 \mathrm{~b})$. Now for any $w^{*} \in W_{0}^{*}$, write $w^{*}=$ $w_{1}^{*}+w_{2}^{*}$, where $w_{1}^{*}, w_{2}^{*} \in W^{*}$ with $\left.w_{1}^{*}\right|_{W^{\prime \prime}}=0$ and $\left.w_{2}^{*}\right|_{W^{\prime}}=0$. Then $\left\langle F^{\prime}(h: m), w^{*}\right\rangle=\left\langle F^{\prime}(h: m), w_{1}^{*}\right\rangle$. Further, $\left\{w^{*} \in W^{*}:\left.w^{*}\right|_{W^{\prime \prime}}=0\right\}$ is spanned by point evaluations $e\left(k_{1}: k_{2}\right)$, where $k_{1}, k_{2} \in K_{M, 1}^{V}$. But $\left\langle F^{\prime}(h: m), e\left(k_{1}: k_{2}\right)\right\rangle=F^{\prime}(h: m)\left(k_{1}: k_{2}\right)$ is a multiple (independent of $m)$ of $\varphi^{\prime}\left(h: k_{1}^{\prime-1} m k_{2}^{\prime-1}\right)$ and $m \rightarrow \varphi^{\prime}\left(h: k_{1}^{\prime-1} m k_{2}^{\prime-1}\right)$ is an element of $\mathscr{V}\left(M^{\prime}: \tau_{1}: \tau_{2}: h\right)$. Thus $F^{\prime}(h) \in \mathscr{S}\left(M^{\prime}: W_{0}: h\right)$. The last assertion follows from Lemma 5.11. Q.E.D.

For $h \in \operatorname{cl}\left(\mathscr{D}_{M^{\prime}}\right)$, write $\mathscr{V}(h)=\mathscr{V}\left(M^{\prime}: \tau_{1}: \tau_{2}: h\right)$. Then each $\mathscr{V}(h)^{*}$ is spanned by the point evaluations $e_{m}(\varphi)=\varphi(m), m \in M^{\prime}, \varphi \in \mathscr{V}(h)$. Now we can associate to each $F \in \mathscr{S}\left(M^{\prime}: W_{0}: h\right)$ an element ${ }^{\prime} F \in \operatorname{Hom}\left(\mathscr{V}(h)^{*}, W_{0}\right)$ so that

$$
{ }^{\prime} F\left(e_{m}\right)=F(m), \quad \text { all } m \in M^{\prime} .
$$

Write

$$
{ }^{\prime} \mathscr{S}(h)=\left\{{ }^{\prime} F: F \in \mathscr{S}\left(M^{\prime}: W_{0}: h\right)\right\} \subseteq \operatorname{Hom}\left(\mathscr{V}(h)^{*}, W_{0}\right) .
$$

For each $h \in \operatorname{cl}\left(\mathscr{D}_{M^{\prime}}\right), K_{M^{\prime}}$ acts on $W_{0}$ by $\left(\tau_{1, h}^{\prime}, \tau_{2, h}^{\prime}\right)$, where

$$
\tau_{i, h}^{\prime}(k)=e^{h}(k)^{\prime} \tau_{i, 0}(p(k)) .
$$


Write $W_{0}(h)$ for $W_{0}$ with this action of $K_{M^{\prime}}$. Note $\operatorname{End}_{K_{M^{\prime}}}\left(W_{0}(h)\right)$ is independent of $h$ so we write simply $\operatorname{End}_{K_{M^{\prime}}}\left(W_{0}\right) . K_{M^{\prime}}$ also acts on $\mathscr{V}(h)$ on the left and right by

$$
\left(k_{1} \cdot \varphi_{h} \cdot k_{2}\right)(m)=\varphi_{h}\left(k_{1}^{-1} m k_{2}^{-1}\right), \quad k_{1}, k_{2} \in K_{M^{\prime}}, m \in M^{\prime}, \varphi_{h} \in \mathscr{V}(h),
$$

and on $\mathscr{V}(h)^{*}$ by

$$
\left(k_{1}^{*} \cdot v^{*} \cdot k_{2}^{*}\right)\left(\varphi_{h}\right)=v^{*}\left(k_{1}^{-1} \cdot \varphi_{h} \cdot k_{2}^{-1}\right) .
$$

Note that

$$
k_{1}^{*} \cdot e_{m} \cdot k_{2}^{*}=e_{k_{1} m k_{2}} \text { for all } k_{1}, k_{2} \in K_{M^{\prime}}, m \in M^{\prime} .
$$

Lemma 5.16. ' $\mathscr{S}(h)=\operatorname{Hom}_{K_{M^{\prime}}}\left(\mathscr{V}(h)^{*}, W_{0}(h)\right)=\left\{T \in \operatorname{Hom}\left(\mathscr{V}(h)^{*}, W_{0}\right)\right.$ : $T\left(k_{1}^{*} \cdot v^{*} \cdot k_{2}^{*}\right)=\tau_{1, h}^{\prime}\left(k_{1}\right) T v^{*} \tau_{2, h}^{\prime \prime}\left(k_{2}\right)$ for all $\left.k_{1}, k_{2} \in K_{M^{\prime}}, v^{*} \in V(h)^{*}\right\}$.

Proof. Because the $e_{m}$ span $\mathscr{V}(h)^{*}$, we have, using (5.15d),

$$
\begin{aligned}
& \operatorname{Hom}_{K_{M^{\prime}}}\left(\mathscr{V}(h)^{*}, W_{0}(h)\right) \\
& =\left\{T \in \operatorname{Hom}\left(\mathscr{V}(h)^{*}, W_{0}\right): T\left(e_{k_{1} m k_{2}}\right)\right. \\
& \left.\quad=\tau_{1, h}^{\prime}\left(k_{1}\right) T\left(e_{m}\right) \tau_{2, h}^{\prime}\left(k_{2}\right) \text { for all } k_{1}, k_{2} \in K_{M^{\prime}}, m \in M^{\prime}\right\} .
\end{aligned}
$$

Now $\operatorname{Hom}\left(\mathscr{V}(h)^{*}, W_{0}\right)$ is the image under $F \rightarrow^{\prime} F$ of all $F: M^{\prime} \rightarrow W_{0}$ satisfying condition (ii) of (5.8c). But now ' $F \in \operatorname{Hom}_{K_{M^{\prime}}}\left(\mathscr{V}(h)^{*}, W_{0}(h)\right)$ if and only if $F\left(k_{1} m k_{2}\right)=\tau_{1, h}^{\prime}\left(k_{1}\right) F(m) \tau_{2, h}^{\prime}\left(k_{2}\right)$, i.e., $F \in \mathscr{S}\left(M^{\prime}: W_{0}: h\right)$. Q.E.D.

Lemma 5.17. $\operatorname{dim} \operatorname{Hom}_{K_{M^{\prime}}}\left(\mathscr{V}(h)^{*}, W_{0}(h)\right)$ is independent of $h \in \operatorname{cl}\left(\mathscr{D}_{M^{\prime}}\right)$.

Proof. By (5.15a), the action of $K_{M^{\prime}}$ on $W_{0}(h)$ is $e^{h}\left(\tau_{1,0}^{\prime}, \tau_{2,0}^{\prime}\right)$. Further, $\mathscr{V}(h)^{*}$ is equivalent as an abstract $K_{M^{\prime}}$-module to

$$
\sum_{\left(\sigma_{1}, \sigma_{2}\right) \in \Sigma_{1}^{\prime} \times \Sigma_{1}^{\prime}} m\left(\sigma_{1, h}\right) m\left(\sigma_{2, h}\right)\left(\sigma_{1,0}^{*} \otimes e^{h}, \sigma_{2, h}\right),
$$

where $m\left(\sigma_{i, h}\right)$ denotes the multiplicity of $\sigma_{i, h}$ in $\left.\pi\left(M^{\prime}: h\right)\right|_{K_{M^{\prime}}}$. But, by $[9,4.9]$, these multiplicities are independent of $h \in \operatorname{cl}\left(\mathscr{D}_{M^{\prime}}\right)$. Thus, there is a finite-dimensional vector space $V_{0}$ on which $K_{M^{\prime}}$ acts on the left and right by $\left(\rho_{1,0}, \rho_{2,0}\right)$ so that $\mathscr{V}(h)^{*}$ is equivalent to $V_{0}(h)$, i.e., $V_{0}$ with the action $e^{h}\left(\rho_{1,0}, \rho_{2,0}\right)$. Thus, for all $h \in \operatorname{cl}\left(\mathscr{D}_{M^{\prime}}\right)$,

$$
\begin{aligned}
\operatorname{dim} \operatorname{Hom}_{K_{M^{\prime}}}\left(\mathscr{V}(h)^{*}, W_{0}(h)\right) & =\operatorname{dim} \operatorname{Hom}_{K_{M^{\prime}}}\left(\mathscr{V}_{0}(h), W_{0}(h)\right) \\
& =\operatorname{dim} \operatorname{Hom}_{K_{M^{\prime}}}\left(\mathscr{V}_{0}(0), W_{0}(0)\right) \text {. Q.E.D. }
\end{aligned}
$$

Fix a matrix coefficient $\psi(0)$ of $\chi(0)$ and write $F\left(\varphi^{\prime}\right)=F\left(\varphi^{\prime}: \psi(0)\right)$, $\varphi^{\prime} \in \mathscr{F}\left(M^{\prime}: \tau_{1}: \tau_{2}\right)$. 
Lemma 5.18. Let $h \in \operatorname{cl}\left(\mathscr{D}_{M^{\prime}}\right)$. Then every $F \in \mathscr{S}\left(M^{\prime}: W_{0}: h\right)$ is a finite linear combination of elements of the form $S F\left(\varphi^{\prime}: h\right)$, where $S \in \operatorname{End}_{K_{M^{\prime}}}\left(W_{0}\right)$, $\varphi^{\prime} \in \mathscr{F}\left(M^{\prime}: \tau_{1}: \tau_{2}\right)$.

Proof. Suppose $\varphi_{1, h}^{\prime}, \ldots, \varphi_{n, h}^{\prime}$ is a basis of $\mathscr{V}(h)$. By $[9,4.4]$ there are holomorphic families $\varphi_{1}^{\prime}, \ldots, \varphi_{n}^{\prime} \in \mathscr{F}\left(M^{\prime}: \tau_{1}: \tau_{2}\right)$ so that $\varphi_{i, h}^{\prime}=\varphi_{i}^{\prime}(h), 1 \leq i \leq$ $n$. Define $\Phi: \mathscr{V}(h)^{*} \rightarrow W_{0}^{n}$ by $\Phi\left(v^{*}\right)=\left({ }^{\prime} F\left(\varphi_{1}^{\prime}: h\right)\left(v^{*}\right), \ldots,{ }^{\prime} F\left(\varphi_{n}^{\prime}: h\right)\left(v^{*}\right)\right)$. Then $\Phi \in \operatorname{Hom}_{K_{M^{\prime}}}\left(\mathscr{V}(h)^{*}, W_{0}(h)^{n}\right)$ since each

$$
{ }^{\prime} F\left(\varphi_{i}^{\prime}: h\right) \in \operatorname{Hom}_{K_{M^{\prime}}}\left(\mathscr{V}(h)^{*}, W_{0}(h)\right) .
$$

Suppose $v^{*} \in \operatorname{Ker} \Phi$. Then ${ }^{\prime} F\left(\varphi_{i}^{\prime}: h\right)\left(v^{*}\right)=0,1 \leq i \leq n$, so, in particular, ${ }^{\prime} F\left(\varphi_{i}^{\prime}: h\right)\left(v^{*}\right)(1: 1)=0,1 \leq i \leq n$. But for all $m \in M^{\prime}$, using (5.12),

$$
{ }^{\prime} F\left(\varphi_{i}^{\prime}: h\right)\left(e_{m}\right)(1: 1)=F\left(\varphi_{i}^{\prime}: h: m\right)(1: 1)=\varphi_{i}^{\prime}(h: m)=e_{m}\left(\varphi_{i, h}^{\prime}\right),
$$

so that ${ }^{\prime} F\left(\varphi_{i}^{\prime}: h\right)\left(v^{*}\right)(1: 1)=v^{*}\left(\varphi_{i, h}^{\prime}\right)$ for all $v^{*} \in \mathscr{V}(h)^{*}$. Thus if $v^{*} \in$ $\operatorname{Ker} \Phi, v^{*}\left(\varphi_{i, h}^{\prime}\right)=0,1 \leq i \leq n$, so that $v^{*}=0$. Thus $\Phi$ is injective.

Decompose $W_{0}^{n}=W_{1} \oplus W_{2}$, where $W_{1}=\Phi\left(\mathscr{V}(h)^{*}\right)$ and $W_{2}=W_{1}^{\perp} \cdot W_{1}$ and $W_{2}$ are $K_{M^{\prime}}$-invariant subspaces, and if we define $\Phi^{-1}: W_{0}^{n} \rightarrow \mathscr{V}(h)^{*}$ by $\left.\Phi^{-1}\right|_{W_{2}}=0$ and $\Phi^{-1} \circ \Phi=$ identity on $\mathscr{V}(h)^{*}$, we have

$$
\Phi^{-1} \in \operatorname{Hom}_{K_{M^{\prime}}}\left(W_{0}(h)^{n}, \mathscr{V}(h)^{*}\right) .
$$

Now for all $F \in \mathscr{S}\left(M^{\prime}: W_{0}: h\right)$, write ${ }^{\prime} F=T \circ \Phi$, where $T={ }^{\prime} F \circ \Phi^{-1} \in$ $\operatorname{Hom}_{K_{M^{\prime}}}\left(W_{0}(h)^{n}, W_{0}(h)\right)$. Let $S_{i} \in \operatorname{Hom}_{K_{M^{\prime}}}\left(W_{0}(h), W_{0}(h)\right)=\operatorname{End}_{K_{M^{\prime}}}\left(W_{0}\right)$ be the restriction of $T$ to the $i$ th component of $W_{0}(h)^{n}$, so that $T\left(w_{1}, \ldots, w_{n}\right)=$ $\sum_{i=1}^{n} S_{i}\left(w_{i}\right)$. Then for all $v^{*} \in \mathscr{V}(h)^{*}$,

$$
\begin{aligned}
{ }^{\prime} F\left(v^{*}\right) & =T \circ \Phi\left(v^{*}\right)=T\left({ }^{\prime} F\left(\varphi_{1}^{\prime}: h\right)\left(v^{*}\right), \ldots,{ }^{\prime} F\left(\varphi_{n}^{\prime}: h\right)\left(v^{*}\right)\right) \\
& =\sum_{i=1}^{n} S_{i}{ }^{\prime} F\left(\varphi_{1}^{\prime}: h\right)\left(v^{*}\right) .
\end{aligned}
$$

Thus $F=\sum_{i=1}^{n} S_{i} F\left(\varphi_{1}^{\prime}: h\right)$. Q.E.D.

Lemma 5.19. Let $h^{\prime} \in \operatorname{cl}\left(\mathscr{D}_{M^{\prime}}\right)$. Then there are a neighborhood $J$ of $h^{\prime}$ in $\operatorname{cl}\left(\mathscr{D}_{M^{\prime}}\right)$ and elements $\varphi_{1}^{\prime}, \ldots, \varphi_{s}^{\prime} \in \mathscr{F}\left(M^{\prime}: \tau_{1}: \tau_{2}\right), S_{1}, \ldots, S_{s} \in \operatorname{End}_{K_{M^{\prime}}}\left(W_{0}\right)$ so that $\left\{S_{i} F\left(\varphi_{i}^{\prime}: h\right)\right\}_{i=1}^{s}$ is a basis for $\mathscr{S}\left(M^{\prime}: W_{0}: h\right)$ for all $h \in J$.

Proof. By Lemma 5.18 elements of the form $\left\{S F\left(\varphi^{\prime}: h^{\prime}\right)\right\}, S \in \operatorname{End}_{K_{M^{\prime}}}\left(W_{0}\right)$, $\varphi^{\prime} \in \mathscr{F}\left(M^{\prime}: \tau_{1}: \tau_{2}\right)$, span $\mathscr{S}\left(M^{\prime}: W_{0}: h^{\prime}\right)$. Thus we can pick $\varphi_{i}^{\prime}, S_{i}$ so that $\left\{S_{i} F\left(\varphi_{i}^{\prime}: h^{\prime}\right)\right\}_{i=1}^{s}$ is a basis for $\mathscr{S}\left(M^{\prime}: W_{0}: h\right)$. But $h \rightarrow S_{i} F\left(\varphi_{i}^{\prime}: h\right)$ is holomorphic, so by the argument used in [9, 4.10], there is a neighborhood $J$ of $h^{\prime}$ in $\operatorname{cl}\left(\mathscr{D}_{M^{\prime}}\right)$ so that $\left\{S_{i} F\left(\varphi_{i}^{\prime}: h\right)\right\}$ are independent for $h \in J$. Now by Lemma 5.17 they are a basis for $\mathscr{S}\left(M^{\prime}: W_{0}: h\right)$. Q.E.D. 
Lemma 5.19 says we can find consistent bases locally in $\mathrm{cl}\left(\mathscr{D}_{M^{\prime}}\right)$. If this was all we needed to do, we could have proved Lemmas 5.16-5.19 for $\mathscr{S}\left(M^{\dagger}: W: h\right)$ instead of $\mathscr{S}\left(M^{\prime}: W_{0}: h\right)$ and had consistent bases of the form $\{S F(\varphi: h)\}, S \in \operatorname{End}_{K_{M^{\dagger}}}(W), \varphi \in \mathscr{F}\left(M^{\dagger}: \tau_{1}: \tau_{2}\right)$. However, when $\operatorname{cl}(\mathscr{D})$ is noncompact, this will not suffice to give a finite open cover with consistent bases. To do this we must use the canonical identifications between spaces of matrix coefficients which exist for holomorphic (or antiholomorphic) relative discrete series representations.

As in [9, 4.11], we can decompose $\mathfrak{m}=\mathfrak{v}_{0} \oplus \mathfrak{m}_{0}^{\prime} \oplus \mathfrak{m}^{\prime \prime}, \mathfrak{v}_{M^{\prime}}=\mathfrak{v}_{0} \oplus \mathfrak{v}^{\prime} \oplus \mathfrak{v}^{\prime \prime}$, and $\mathscr{D}_{M^{\prime}}=i \mathfrak{v}_{0}^{*} \times \mathscr{D}^{\prime} \times \mathscr{D}^{\prime \prime}$ in such a way that $\operatorname{cl}\left(\mathscr{D}^{\prime}\right) \subseteq i\left(\mathfrak{v}^{\prime}\right)^{*}$ is compact, and $\mathscr{D}^{\prime \prime}=$ $\prod_{i=1}^{k} \mathscr{D}_{i} \subseteq i\left(\mathfrak{v}^{\prime \prime}\right)^{*}$ is the product of open half-lines $\mathscr{D}_{i} \subseteq i \mathfrak{v}_{i}^{*}$, where $\mathfrak{v}_{i}=\mathfrak{v}^{\prime \prime} \cap \mathfrak{m}_{i}$ and the $\mathfrak{m}_{i}$ are the simple ideals of $\mathfrak{m}^{\prime \prime}$. The $\mathfrak{m}_{i}$ are necessarily noncompact and of hermitian type, and the $\mathscr{D}_{i}$ correspond to families of holomorphic or antiholomorphic relative discrete series of $\mathfrak{m}_{i}$.

Let $I$ be any subset of $\{1, \ldots, k\}$. Corresponding to $I$ we define a family $\mathscr{F}_{I}\left(M^{\prime}: \tau_{1}: \tau_{2}\right)$ as in $[9,4.6]$, except that for $i \in I$ we take $\mathscr{F}_{I, i}\left(M^{\prime}: \tau_{1}: \tau_{2}\right)$ to be the linear span of the one-parameter families of matrix coefficients based on holomorphic sections $[8,6.15]$ rather than the linear span of the holomorphic families of matrix coefficients based on the highest weight module structure [8, 6.9]. Thus $\mathscr{F}_{\varnothing}=\mathscr{F}$. Note Lemmas 5.18 and 5.19 are still valid for $\mathscr{F}_{I}$ in place of $\mathscr{F}$.

For each $1 \leq i \leq k$, write $\operatorname{cl}\left(\mathscr{D}_{i}\right)=\mathscr{D}_{i}^{c} \cup \mathscr{D}_{i}^{n}$, where $\mathscr{D}_{i}^{c}$ and $\mathscr{D}_{i}^{n}$ are intervals, relatively open in $\operatorname{cl}\left(\mathscr{D}_{i}\right)$, so that $\operatorname{cl}\left(\mathscr{D}_{i}^{c}\right)$ is compact and $\mathscr{D}_{i}^{n}$ is an open half-line with $\operatorname{cl}\left(\mathscr{D}_{i}{ }^{n}\right) \subseteq \mathscr{D}_{i}$. For each $I \subseteq\{1, \ldots, k\}$ define a relatively open set in $\operatorname{cl}\left(\mathscr{D}_{M^{\prime}}\right)$ by

$$
\mathscr{O}_{I}=i \mathfrak{v}_{0}^{*} \times \operatorname{cl}\left(\mathscr{D}^{\prime}\right) \times \prod_{i \notin I} \mathscr{D}_{i}^{c} \times \prod_{i \in I} \mathscr{D}_{i}^{n}
$$

Clearly $\left\{\mathscr{O}_{I}\right\}$ is an open cover of $\operatorname{cl}\left(\mathscr{D}_{M^{\prime}}\right)$. Further, by $[8,6.16]$, for each $\varphi \in \mathscr{F}_{I, i}\left(M^{\prime}: \tau_{1}: \tau_{2}\right)$, there are $\left\{\varphi_{j}\right\}$ in $\mathscr{F}_{i}\left(M^{\prime}: \tau_{1}: \tau_{2}\right)$ and rational functions $\left\{r_{j}\right\}$ on $\left(\mathfrak{v}_{i}\right)_{\mathrm{C}}^{*}$ with no poles in $\mathscr{D}_{i}$, so that $\varphi(h)=\sum_{j} r_{j}(h) \varphi_{j}(h)$. Thus given $\varphi \in \mathscr{F}_{I}\left(M^{\prime}: \tau_{1}: \tau_{2}\right)$, there are $\varphi_{j} \in \mathscr{F}\left(M^{\prime}: \tau_{1}: \tau_{2}\right)$ and rational functions $\left\{r_{j}\right\}$ on $\left(\mathfrak{v}_{M^{\prime}}\right)_{\mathrm{C}}^{*}$ with no poles in $\operatorname{cl}\left(\mathscr{O}_{I}\right)$ so that

$$
\varphi(h)=\sum_{j} r_{j}(h) \varphi_{j}(h), \quad h \in\left(\mathfrak{v}_{M^{\prime}}\right)_{\mathbf{C}}^{*}
$$

Now fix any $b_{0} \in i v_{0}^{*}, b_{i} \in \mathscr{D}_{i}^{n}, i \in I$. We define a compact subset $\Omega_{I}$ of $\operatorname{cl}\left(\mathscr{O}_{I}\right)$ by

$$
\Omega_{I}=\left\{b_{0}\right\} \times \operatorname{cl}\left(\mathscr{D}^{\prime}\right) \times \prod_{i \notin I} \operatorname{cl}\left(\mathscr{D}_{i}^{c}\right) \times \prod_{i \in I}\left\{b_{i}\right\}
$$


Define $\alpha_{I}: \operatorname{cl}\left(\mathscr{O}_{I}\right) \rightarrow \Omega_{I}$ by

$$
\alpha_{I}\left(h_{0}, h^{\prime}, \sum_{i \notin I} h_{i}, \sum_{i \in I} h_{i}\right)=\left(b_{0} ; h^{\prime} ; \sum_{i \notin I} h_{i}, \sum_{i \in I} b_{i}\right),
$$

where $h_{0} \in i v_{0}^{*}, h^{\prime} \in \operatorname{cl}\left(\mathscr{D}^{\prime}\right), h_{i} \in \operatorname{cl}\left(\mathscr{D}_{i}^{c}\right), i \notin I$, and $h_{i} \in \operatorname{cl}\left(\mathscr{D}_{i}^{n}\right), i \in I$.

Lemma 5.23. Fix $h \in \operatorname{cl}\left(\mathscr{O}_{I}\right)$ and write $b=\alpha_{I}(h)$. There is an isomorphism $T_{h}: \mathscr{V}\left(M^{\prime}: \tau_{1}: \tau_{2}: h\right) \rightarrow \mathscr{V}\left(M^{\prime}: \tau_{1}: \tau_{2}: b\right)$ so that

(i) $T_{h}\left(\varphi^{\prime}(h)\right)=\varphi^{\prime}(b)$ for all $\varphi^{\prime} \in \mathscr{F}_{I}\left(M^{\prime}: \tau_{1}: \tau_{2}\right)$;

(ii) $T_{h}\left(k_{1} \cdot \varphi_{h}^{\prime} \cdot k_{2}\right)=e^{h-b}\left(k_{1}^{-1} k_{2}^{-1}\right)\left(k_{1} \cdot T_{h}\left(\varphi_{h}^{\prime}\right) \cdot k_{2}\right)$ for all $k_{1}, k_{2} \in K_{M^{\prime}}$, $\varphi_{h}^{\prime} \in \mathscr{V}\left(M^{\prime}: \tau_{1}: \tau_{2}: h\right)$.

Proof. Since the spaces $\mathscr{V}(h)=\mathscr{V}\left(M^{\prime}: \tau_{1}: \tau_{2}: h\right)$ and $\mathscr{F}_{I}=\mathscr{F}_{I}\left(M^{\prime}: \tau_{1}: \tau_{2}\right)$ are products over the simple factors of $M^{\prime}$, it suffices to define $T_{h}$ on each simple factor. So we may as well assume that $M^{\prime}$ is simple or a vector group. Also, $\alpha_{I}$ is the identity map unless $\mathscr{D}_{M^{\prime}}=i \mathrm{v}_{0}^{*}$ or $\mathscr{D}_{M^{\prime}}=\mathscr{D}_{i}, i \in I$. Suppose first that $M^{\prime}=V_{0}$ is a vector group. For all $h \in i \mathrm{v}_{0}^{*}, \mathscr{V}(h)=\mathbf{C} e^{h}$. Define $T_{h}: \mathscr{V}(h) \rightarrow \mathscr{V}(b)$ by $T_{h}\left(c e^{h}\right)=c e^{b}$. Now $\mathscr{F}_{I}=\mathbf{C} \varphi_{0}^{\prime}$, where $\varphi_{0}^{\prime}(h)=e^{h}$ for all $h \in i \mathfrak{v}_{0}^{*}$. Thus $T_{h}\left(c \varphi_{0}^{\prime}(h)\right)=c \varphi_{0}^{\prime}(b)$. Further, $k_{1} \cdot e^{h} \cdot k_{2}=e^{h}\left(k_{1}^{-1} k_{2}^{-1}\right) e^{h}$ for all $h \in i \mathfrak{v}_{0}^{*}, k_{1}, k_{2} \in K_{M^{\prime}}$, so that

$$
T_{h}\left(k_{1} \cdot c e^{h} \cdot k_{2}\right)=e^{h}\left(k_{1}^{-1} k_{2}^{-1}\right) T_{h}\left(c e^{h}\right)=e^{h-b}\left(k_{1}^{-1} k_{2}^{-1}\right) k_{1} \cdot T_{h}\left(c e^{h}\right) \cdot k_{2} .
$$

Thus (i) and (ii) are satisfied for $T_{h}$. Now suppose $M^{\prime}=M_{i}$ for some $i \in I$. We use the notation of [7, §3]. There are $K_{M^{\prime}}$-finite holomorphic families $\left\{f_{i, h}\right\},\left\{g_{j, h}\right\}$ and corresponding one-parameter families of matrix coefficients $\varphi_{i, j}^{\prime} \in \mathscr{F}_{I}, \varphi_{i, j}^{\prime}(h: m)=\left\langle L(m) f_{i, h}, g_{j, h}\right\rangle$, so that $\left\{\varphi_{i, j}^{\prime}(h)\right\}$ is a basis for $\mathscr{V}(h)$ for all $h \in \mathscr{D}_{i}$. Define $T_{h}: \mathscr{V}(h) \rightarrow \mathscr{V}(b)$ by $T_{h}\left(\varphi_{i, j}^{\prime}(h)\right)=\varphi_{i, j}^{\prime}(b)$, all $i, j$. Now $\left\{f_{i, h}\right\},\left\{g_{j, h}\right\}$ correspond to $K_{M^{\prime}}$-finite holomorphic vectorvalued functions $\left\{F_{i}\right\},\left\{G_{j}\right\}$ on $M^{\prime} / K_{M^{\prime}}$ as in [7, 3.10]. Let $U, U^{\prime}$ be the $K_{M^{\prime}}$-invariant spaces of functions spanned by the $\left\{F_{i}\right\},\left\{G_{j}\right\}$ respectively, and let $\left\{f_{i}^{*}\right\},\left\{g_{j}^{*}\right\}$ denote the corresponding dual bases of $U^{*},\left(U^{\prime}\right)^{*}$. Now an arbitrary $\varphi^{\prime} \in \mathscr{F}_{I}$ is of the form $\varphi^{\prime}(h: x)=\left\langle L(m) f_{h}, g_{h}\right\rangle$, where $f_{h}, g_{h}$ are $K_{M^{\prime}}$-finite families of holomorphic sections based on $f \in U, g \in U^{\prime}$. Now using [7, 3.11], for all $k_{i} \in K_{M^{\prime}}, h \in \mathscr{D}_{i}$,

$$
L\left(k_{2}^{-1}\right) f_{h}=e^{h}\left(k_{2}^{-1}\right) \sum_{i}\left\langle L\left(k_{2}^{-1}\right) f, f_{i}^{*}\right\rangle f_{i, h},
$$

and

$$
L\left(k_{1}\right) g_{h}=e^{h}\left(k_{1}\right) \sum_{j}\left\langle L\left(k_{1}\right) g, g_{j}^{*}\right\rangle g_{j, h}
$$


Thus for all $h \in \mathscr{D}_{i}$,

$$
\begin{aligned}
\left(k_{1} \cdot \varphi^{\prime}(h) \cdot k_{2}\right)(m) & =\varphi^{\prime}\left(h: k_{1}^{-1} m k_{2}^{-1}\right)=\left\langle L(m) L\left(k_{2}^{-1}\right) f_{h}, L\left(k_{1}\right) g_{h}\right\rangle \\
& =e^{h}\left(k_{1}^{-1} k_{2}^{-1}\right) \sum_{i, j}\left\langle L\left(k_{2}^{-1}\right) f, f_{i}^{*}\right\rangle \overline{\left\langle L\left(k_{1}\right) g, g_{j}^{*}\right\rangle} \varphi_{i, j}^{\prime}(h: m) .
\end{aligned}
$$

Thus

$$
\begin{aligned}
T_{h}\left(k_{1} \cdot \varphi^{\prime}(h) \cdot k_{2}\right) & =e^{h}\left(k_{1}^{-1} k_{2}^{-1}\right) \sum_{i, j}\left\langle L\left(k_{2}^{-1}\right) f, f_{i}^{*}\right\rangle \overline{\left\langle L\left(k_{1}\right) g, g_{j}^{*}\right\rangle} \varphi_{i, j}^{\prime}(b: m) \\
& =e^{h-b}\left(k_{1}^{-1} k_{2}^{-1}\right) k_{1} \cdot \varphi^{\prime}(b) \cdot k_{2} .
\end{aligned}
$$

Taking $k_{1}=k_{2}=1$, this proves that $T_{h}\left(\varphi^{\prime}(h)\right)=\varphi^{\prime}(b)$. But for each $\varphi_{h}^{\prime} \in$ $\mathscr{V}(h)$, there is $\varphi^{\prime} \in \mathscr{F}_{I}$ with $\varphi^{\prime}(h)=\varphi_{h}^{\prime}$. Then $T_{h}\left(\varphi_{h}^{\prime}\right)=\varphi^{\prime}(b)$ so we have also established (ii). Q.E.D.

We will use the isomorphism $T_{h}: \mathscr{V}\left(M^{\prime}: \tau_{1}: \tau_{2}: h\right) \rightarrow \mathscr{V}\left(M^{\prime}: \tau_{1}: \tau_{2}: b\right)$, $b=\alpha_{I}(h)$, to define an isomorphism $T_{h}^{\prime}: \mathscr{S}\left(M^{\prime}: W_{0}: h\right) \rightarrow \mathscr{S}\left(M^{\prime}: W_{0}: b\right)$. Write $\mathscr{V}(h)=\mathscr{V}\left(M^{\prime}: \tau_{1}: \tau_{2}: h\right)$ for all $h \in\left(\mathfrak{v}_{M^{\prime}}\right)_{\mathbf{C}}^{*}$. We can identify $\mathscr{S}\left(M^{\prime}: W_{0}: h\right)$ with

$\left(\mathscr{V}(h) \otimes W_{0}(h)\right)^{K_{M^{\prime}}}$

$$
\begin{aligned}
=\left\{\sum_{i} \varphi_{i} \otimes w_{i} \in \mathscr{V}(h) \otimes W_{0}: \sum_{i}\left(k_{1}^{-1} \cdot \varphi_{i} \cdot k_{2}^{-1}\right) \otimes w_{i}\right. \\
\left.=\sum_{i} \varphi_{i} \otimes \tau_{1, h}^{\prime}\left(k_{1}\right) w_{i} \tau_{2, h}^{\prime}\left(k_{2}\right) \text { for all } k_{1}, k_{2} \in K_{M^{\prime}}\right\} .
\end{aligned}
$$

Define $T_{h}^{\prime}: \mathscr{V}(h) \otimes W_{0} \rightarrow \mathscr{V}(b) \otimes W_{0}$ by $T_{h}^{\prime}\left(\sum \varphi_{i} \otimes w_{i}\right)=\sum T_{h}\left(\varphi_{i}\right) \otimes w_{i}:$ It is an isomorphism onto since $T_{h}: \mathscr{V}(h) \rightarrow \mathscr{V}(b)$ is an isomorphism onto.

Lemma 5.24. $T_{h}^{\prime}\left(\mathscr{V}(h) \otimes W_{0}(h)\right)^{K_{M^{\prime}}}=\left(\mathscr{V}(b) \otimes W_{0}(b)\right)^{K_{M^{\prime}}}$.

Proof. Let $\sum \varphi_{i} \otimes w_{i} \in\left(\mathscr{V}(h) \otimes W_{0}(h)\right)^{K_{M^{\prime}}}$. Then for all $k_{1}, k_{2} \in K_{M^{\prime}}$, using Lemma 5.23(ii),

$$
\begin{aligned}
\sum_{i} k_{1}^{-1} \cdot T_{h}\left(\varphi_{i}\right) \cdot k_{2}^{-1} \otimes w_{i} & =\sum_{i} e^{b-h}\left(k_{1} k_{2}\right) \sum_{i} T_{h}\left(k_{1}^{-1} \cdot \varphi_{i} \cdot k_{2}^{-1}\right) \otimes w_{i} \\
& =e^{b-h}\left(k_{1} k_{2}\right) T_{h}^{\prime}\left(\sum_{i} k_{1}^{-1} \cdot \varphi_{i} \cdot k_{2}^{-1} \otimes w_{i}\right) \\
& =e^{b-h}\left(k_{1} k_{2}\right) T_{h}^{\prime}\left(\sum_{i} \varphi_{i} \otimes \tau_{1, h}^{\prime}\left(k_{1}\right) w_{i} \tau_{2, h}^{\prime}\left(k_{2}\right)\right) \\
& =T_{h}^{\prime}\left(\sum_{i} \varphi_{i} \otimes \tau_{1, b}^{\prime}\left(k_{1}\right) w_{i} \tau_{2, b}^{\prime}\left(k_{2}\right)\right) \\
& =\sum_{i} T_{h}\left(\varphi_{i}\right) \otimes \tau_{1, b}^{\prime}\left(k_{1}\right) w_{i} \tau_{2, b}^{\prime}\left(k_{2}\right) .
\end{aligned}
$$

Thus $T_{h}^{\prime}\left(\sum \varphi_{i} \otimes w_{i}\right) \in\left(\mathscr{V}(b) \otimes W_{0}(b)\right)^{K_{M^{\prime}}}$. Q.E.D. 
Lemma 5.25. Suppose $F\left(\varphi^{\prime}: h\right)$ is defined as in equation (5.12) when $\varphi^{\prime} \in \mathscr{F}_{I}\left(M^{\prime}: \tau_{1}: \tau_{2}\right)$ and let $S \in \operatorname{End}_{K_{M^{\prime}}}\left(W_{0}\right)$. Then $T_{h}^{\prime}\left(S F\left(\varphi^{\prime}: h\right)\right)=$ $S F\left(\varphi^{\prime}: b\right)$, where $b=\alpha_{I}(h)$.

Proof. Recall $F\left(\varphi^{\prime}: h: m\right) \in W_{0} \cap W^{\prime}$ for all $h, m$. Pick a basis for $\left(W_{0} \cap W^{\prime}\right)^{*}$ consisting of point evaluations $e_{i}=e\left(k_{i}, b_{i}\right), k_{i}, b_{i} \in K_{M, 1}^{V}$. Let $\left\{w_{i}\right\}$ denote the corresponding dual basis of $W_{0} \cap W^{\prime}$. Then $F\left(\varphi^{\prime}: h\right)$ corresponds to $\sum_{i} e_{i}\left(F\left(\varphi^{\prime}: h\right)\right) \otimes w_{i}$ in $\mathscr{V}(h) \otimes W_{0}$. Now by formula $(5.12)$,

$$
e_{i}\left(F\left(\varphi^{\prime}: h\right)\right)=c_{i} e^{h}\left(k_{i}^{\prime} b_{i}^{\prime}\right) k_{i}^{\prime} \cdot \varphi^{\prime}(h) \cdot b_{i}^{\prime}, \quad \text { where } c_{i} \in \mathbf{C}, k_{i}^{\prime}, b_{i}^{\prime} \in K_{M^{\prime}} .
$$

Thus,

$$
S F\left(\varphi^{\prime}: h\right)=\sum_{i} c_{i} e^{h}\left(k_{i}^{\prime} b_{i}^{\prime}\right) k_{i}^{\prime} \cdot \varphi^{\prime}(h) \cdot b_{i}^{\prime} \otimes S w_{i}
$$

so

$$
\begin{aligned}
T_{h}^{\prime}\left(S F\left(\varphi^{\prime}: h\right)\right) & =\sum_{i} c_{i} e^{h}\left(k_{i}^{\prime} b_{i}^{\prime}\right) T_{h}\left(k_{i}^{\prime} \cdot \varphi^{\prime}(h) \cdot b_{i}^{\prime}\right) \otimes S w_{i} \\
& =\sum_{i} c_{i} e^{b}\left(k_{i}^{\prime} b_{i}^{\prime}\right) k_{i}^{\prime} \cdot \varphi^{\prime}(b) \cdot b_{i}^{\prime} \otimes S w_{i}=S F\left(\varphi^{\prime}: b\right)
\end{aligned}
$$

using (i) and (ii) of Lemma 5.23. Q.E.D.

Lemma 5.26. There is a finite open cover $\mathscr{J}_{I}$ of $\mathscr{O}_{I}$ satisfying the following. For each $J \in \mathscr{J}_{I}$ there are $\varphi_{1}^{\prime}, \ldots, \varphi_{s}^{\prime} \in \mathscr{F}_{I}\left(M^{\prime}: \tau_{1}: \tau_{2}\right)$ and $S_{1}, \ldots, S_{s} \in$ $\operatorname{End}_{K_{M^{\prime}}}\left(W_{0}\right)$ so that $\left\{S_{i} F\left(\varphi_{i}^{\prime}: h\right)\right\}_{i=1}^{s}$ is a basis for $\mathscr{S}\left(M^{\prime}: W_{0}: h\right)$ for all $h \in J$.

Proof. Using Lemma 5.19 we can find a finite open cover $\widetilde{J_{I}}$ of $\Omega_{I}$ so that on each $\tilde{J} \in \widetilde{J_{I}}$ we have consistent bases of the form $\left\{S_{i} F\left(\varphi_{i}^{\prime}: b\right)\right\}, b \in \widetilde{J}$. Now take $\mathscr{J}_{I}=\left\{\alpha_{I}^{-1}(\widetilde{J}) \cap \mathscr{O}_{I}: \widetilde{J} \in \widetilde{\mathscr{J}}_{I}\right\} . \mathscr{J}_{I}$ is an open cover of $\mathscr{O}_{I}$ and for each $h \in J$, by Lemma 5.24 and Lemma 5.25, $\left\{\left(T_{h}^{\prime}\right)^{-1}\left(S_{i} F\left(\varphi_{i}^{\prime}: b\right)\right)\right\}_{i=1}^{s}=$ $\left\{S_{i} F\left(\varphi_{i}^{\prime}: h\right)\right\}_{i=1}^{s}$ is a basis for $\mathscr{S}\left(M^{\prime}: W_{0}: h\right)$ whenever $\left\{S_{i} F\left(\varphi_{i}^{\prime}: b\right)\right\}_{i=1}^{s}$ is a basis for $\mathscr{S}\left(M^{\prime}: W_{0}: b\right)$, where $b=\alpha_{I}(h)$. Q.E.D.

We now turn to the problem of finding good consistent bases for the spaces $\mathscr{S}\left(M^{\prime}: W_{0}: h\right)^{*}, h \in \mathscr{O}_{I}$. For each $i \in I$, let $\psi_{i} \in \mathscr{F}_{I, i}=$ $\mathscr{F}_{I, i}\left(M^{\prime}: \tau_{1}: \tau_{2}\right)$. Thus $\psi_{i}$ is a one-parameter family of matrix coefficients based on holomorphic sections for the group $M_{i}$. Pick $m^{\prime} \in M^{\prime}(I)=M_{0}^{\prime} \times$ $\prod_{i \notin I} M_{i}$, and let $1 \in V_{0}=\exp \left(\mathfrak{v}_{0}\right)$ be the identity element. For $h \in \operatorname{cl}\left(\mathscr{O}_{I}\right)$, we define $\delta(h)=\delta\left(m^{\prime}: \prod_{i \in I} \psi_{i}: h\right) \in \mathscr{V}(h)^{*}$ by

$$
\begin{array}{r}
\langle\delta(h), \varphi\rangle=\int_{\prod_{i \in I} M_{i} / Z_{i}} \varphi\left(1: m^{\prime}: \prod_{i \in I} x_{i}\right) \prod_{i \in I} \overline{\psi_{i}\left(h_{i}: x_{i}\right)} \prod_{i \in I} d\left(x_{i} Z\right), \\
\varphi \in \mathscr{V}(h) .
\end{array}
$$


That is, $\delta(h)$ is evaluation of $\varphi$ at a point in $V_{0} \times M^{\prime}(I)$, together with the $L_{2}$ inner product with $\psi_{i}\left(h_{i}\right)$ for each of the variables in $M_{i}, i \in I$. Here $h_{i}$ denotes the component of $h$ in $i v_{i}^{*}, i \in I$, and $Z_{i}=V_{i} \cap Z_{M_{i}}$ is a central subgroup of $M_{i}$. Define

$$
\mathscr{F}_{I}^{*}=\left\{\delta\left(m^{\prime}: \prod_{i \in I} \psi_{i}\right): m^{\prime} \in M^{\prime}(I), \psi_{i} \in \mathscr{F}_{I, i}, i \in I\right\} .
$$

For each fixed $h \in \operatorname{cl}\left(\mathscr{O}_{I}\right),\left\{\delta(h): \delta \in \mathscr{F}_{I}^{*}\right\}$ separates points in $\mathscr{V}(h)$, and so spans $\mathscr{V}(h)^{*}$.

Lemma 5.28. Let $h \in \operatorname{cl}\left(\mathscr{O}_{I}\right)$ and write $b=\alpha_{I}(h)$. Then there is an isomorphism $L_{h}: \mathscr{V}(h)^{*} \rightarrow \mathscr{V}(b)^{*}$ so that

(i) $L_{h}(\delta(h))=\delta(b)$ for all $\delta \in \mathscr{F}_{I}^{*}$;

(ii) $L_{h}\left(k_{1}^{*} \cdot v^{*} \cdot k_{2}^{*}\right)=e^{h-b}\left(k_{1} k_{2}\right) k_{1}^{*} \cdot L_{h}\left(v^{*}\right) \cdot k_{2}^{*}$ for all $v^{*} \in \mathscr{V}(h)^{*}, k_{1}, k_{2} \in$ $K_{M^{\prime}}$.

Proof. As in Lemma 5.23, it suffices to prove the lemma when $M^{\prime}=V_{0}$ is a vector group or $M^{\prime}=M_{i}, i \in I$. Suppose first that $M^{\prime}=V_{0}$. Then $\mathscr{V}(h)=\mathbf{C} e^{h}$, and the only $\delta \in \mathscr{F}_{I}^{*}$ is given by $\delta(h)\left(c e^{h}\right)=c$. Now $\mathscr{V}(h)^{*}=$ $\mathbf{C} \delta(h)$ for all $h \in i \mathfrak{v}_{0}^{*}$, so we can define $L_{h}$ by $L_{h}(\delta(h))=\delta(b)$. Further, $k_{1}^{*} \cdot \delta(h) \cdot k_{2}^{*}=e^{h}\left(k_{1} k_{2}\right) \delta(h)$ for all $k_{1}, k_{2} \in K_{M^{\prime}}, h \in i \mathfrak{v}_{0}^{*}$. Thus

$$
L_{h}\left(k_{1}^{*} \cdot \delta(h) \cdot k_{2}^{*}\right)=e^{h}\left(k_{1} k_{2}\right) \delta(b)=e^{h-b}\left(k_{1} k_{2}\right) k_{1}^{*} \cdot L_{h}(\delta(h)) \cdot k_{2}^{*},
$$

so that $L_{h}$ satisfies (ii).

If $M^{\prime}=M_{i}, i \in I$, define the basis $\left\{\varphi_{p q}(h)\right\}$ of $\mathscr{V}(h), h \in \mathscr{D}_{i}$, as in Lemma 5.23. Then $\left\{\delta_{p q}(h)=\delta\left(\varphi_{p q}: h\right)\right\}$ gives a basis for $\mathscr{V}(h)^{*}$ for all $h \in\left(\mathscr{D}_{i}{ }^{n}\right) \subseteq \mathscr{D}_{i}$. Thus we can define an isomorphism $L_{h}: \mathscr{V}(h)^{*} \rightarrow \mathscr{V}(b)^{*}$ by $L_{h}\left(\delta_{p q}(h)\right)=\delta_{p q}(b)$. Now an arbitrary family $\delta \in \mathscr{F}_{I}^{*}$ is of the form $\delta(h)=\delta(\psi: h), \psi \in \mathscr{F}_{I, i}$. But as in Lemma 5.23, $\psi(h)=\sum c_{p q} \varphi_{p q}(h)$ is a linear combination of the families $\varphi_{p q}$, so $\delta(\psi: h)=\sum_{p q} \overline{c_{p q}} \delta_{p q}(h)$ for all $h \in$ $\operatorname{cl}\left(\mathscr{D}_{i}^{n}\right)$, and $L_{h}(\delta(\psi: h))=\delta(\psi: b)$. Further, given $k_{1}, k_{2} \in K_{M^{\prime}}$, there are constants $c_{p q}\left(k_{1}: k_{2}\right)$ so that $k_{1} \cdot \psi(h) \cdot k_{2}=e^{h}\left(k_{1}^{-1} k_{2}^{-1}\right) \sum c_{p q}\left(k_{1}: k_{2}\right) \varphi_{p q}(h)$, so that

$$
k_{1}^{*} \cdot \delta(\psi: h) \cdot k_{2}^{*}=\overline{e^{h}\left(k_{1}^{-1} k_{2}^{-1}\right)} \sum_{p q} \overline{c_{p q}\left(k_{1}: k_{2}\right)} \delta_{p q}(h) .
$$

Thus

$$
L_{h}\left(k_{1}^{*} \cdot \delta(\psi: h) \cdot k_{2}^{*}\right)=e^{h-b}\left(k_{1} k_{2}\right) k_{1}^{*} \cdot L_{h}(\delta(h)) \cdot k_{2}^{*} \text {. Q.E.D. }
$$

Recall $\mathscr{S}\left(M^{\prime}: W_{0}: h\right)=\left(\mathscr{V}(h) \otimes W_{0}(h)\right)^{K_{M^{\prime}}}$. Thus each element $\sum_{i} v_{i}^{*} \otimes$ $w_{i}^{*} \in \mathscr{V}(h)^{*} \otimes W_{0}^{*}$ gives an element $R_{h}\left(\sum_{i} v_{i}^{*} \otimes w_{i}^{*}\right)$ of $\mathscr{S}\left(M^{\prime}: W_{0}: h\right)^{*}$ by restriction to $\left(\mathscr{V}(h) \otimes W_{0}(h)\right)^{K_{M^{\prime}}}$. Let $\left(\mathscr{V}(h)^{*} \otimes W_{0}^{*}(h)\right)^{K_{M^{\prime}}}=\left\{\sum_{i} v_{1}^{*} \otimes w_{i}^{*} \in\right.$ $\mathscr{V}(h)^{*} \otimes W_{0}^{*}: \sum_{i} k_{1}^{*} \cdot v_{i}^{*} \cdot k_{2}^{*} \otimes w_{i}^{*}=\sum_{i} v_{i}^{*} \otimes \tau_{1, h}^{*}\left(k_{1}^{-1}\right) w_{i}^{*} \tau_{2, h}^{*}\left(k_{2}^{-1}\right)$ for all $\left.k_{1}, k_{2} \in K_{M^{\prime}}\right\}$. 
Lemma 5.29. $R_{h}:\left(\mathscr{V}(h)^{*} \otimes W_{0}^{*}(h)\right)^{K_{M^{\prime}}} \rightarrow\left(\left(\mathscr{V}(h) \otimes W_{0}(h)\right)^{K_{M^{\prime}}}\right)^{*}$ is an isomorphism.

Proof. Write $U(h)=\mathscr{V}(h) \otimes W_{0}(h), U(h)^{*}=\left(\mathscr{V}(h) \otimes W_{0}(h)\right)^{*} \cong \mathscr{V}(h)^{*} \otimes$ $W_{0}(h)^{*}$. Then $U(h), U(h)^{*}$ are finite dimensional Hilbert spaces on which $K_{M^{\prime}}$ acts by unitary double representations $\left(\rho_{1}(h), \rho_{2}(h)\right),\left(\rho_{1}^{*}(h), \rho_{2}^{*}(h)\right)$ given by

$$
\rho_{1}\left(h: k_{1}\right)(\varphi \otimes w) \rho_{2}\left(h: k_{2}\right)=k_{1} \cdot \varphi \cdot k_{2} \otimes \tau_{1, h}^{\prime}\left(k_{1}\right) w \tau_{2, h}^{\prime}\left(k_{2}\right)
$$

and

$$
\rho_{1}^{*}\left(h: k_{1}\right)\left(v^{*} \otimes w^{*}\right) \rho_{2}^{*}\left(h: k_{2}\right)=k_{1}^{*} \cdot v^{*} \cdot k_{2}^{*} \otimes \tau_{1, h}^{*}\left(k_{1}\right) w^{*} \tau_{2, h}^{*}\left(k_{2}\right) ;
$$

$\left(\mathscr{V}(h) \otimes W_{0}(h)\right)^{K_{M^{\prime}}}=U(h)^{K_{M^{\prime}}}$ and $\left(\mathscr{V}(h)^{*} \otimes W_{0}^{*}\right)^{K_{M^{\prime}}}=\left(U(h)^{*}\right)^{K_{M^{\prime}}}$ are the spaces of $K_{M^{\prime}}$-fixed vectors for these representations. We must show $R_{h}$ : $\left(U(h)^{*}\right)^{K_{M^{\prime}}} \rightarrow\left(U(h)^{K_{M^{\prime}}}\right)^{*}$ is an isomorphism. Define the conjugate linear isomorphism $T: U(h) \rightarrow U(h)^{*}$ by $T u=\langle\cdot, u\rangle, u \in U(h)$. Since $\left(\rho_{1}(h), \rho_{2}(h)\right)$ is unitary,

$$
T\left(\rho_{1}\left(h: k_{1}\right) u \rho_{2}\left(h: k_{2}\right)\right)=\rho_{1}^{*}\left(h: k_{1}\right) T u \rho_{2}^{*}\left(h: k_{2}\right) \quad \text { for all } k_{1}, k_{2} \in K_{M^{\prime}} .
$$

Thus $T\left(U(h)^{K_{M^{\prime}}}\right)=\left(U(h)^{*}\right)^{K_{M^{\prime}}}$. In particular,

$$
\operatorname{dim}\left(U(h)^{*}\right)^{K_{M^{\prime}}}=\operatorname{dim} U(h)^{K_{M^{\prime}}}=\operatorname{dim}\left(U(h)^{K_{M^{\prime}}}\right)^{*},
$$

so it is enough to show that $R_{h}$ is injective. Let $u^{*} \in\left(U(h)^{*}\right)^{K_{M^{\prime}}}$ with $R_{h}\left(u^{*}\right)=$ 0 . Write $u^{*}=T u, u \in U(h)^{K_{M^{\prime}}}$. Then $0=R_{h}\left(u^{*}\right)(u)=\langle u, u\rangle$, so $u=0$, and $u^{*}=T u=0$. Q.E.D.

By the lemma we can define an injection

$$
R_{h}^{-1}:\left(\left(\mathscr{V}(h) \otimes W_{0}(h)\right)^{K_{M^{\prime}}}\right)^{*} \rightarrow\left(\mathscr{V}(h)^{*} \otimes W_{0}(h)^{*}\right)^{K_{M^{\prime}}} \subseteq \mathscr{V}(h)^{*} \otimes W_{0}^{*}
$$

so that $R_{h} \circ R_{h}^{-1}$ is the identity on $\left(\left(\mathscr{V}(h) \otimes W_{0}(h)\right)^{K_{M^{\prime}}}\right)^{*}=\mathscr{S}\left(M^{\prime}: W_{0}: h\right)^{*}$. Define $L_{h}^{\prime}: \mathscr{S}\left(M^{\prime}: W_{0}: h\right)^{*} \rightarrow \mathscr{S}\left(M^{\prime}: W_{0}: b\right)^{*}$ by

$$
L_{h}^{\prime}=R_{b} \circ\left(L_{h} \otimes 1\right) \circ R_{h}^{-1},
$$

where $L_{h} \otimes 1: \mathscr{V}(h)^{*} \otimes W_{0}^{*} \rightarrow \mathscr{V}(b)^{*} \otimes W_{0}^{*}$.

Lemma 5.31. $L_{h}^{\prime}$ is an isomorphism of $\mathscr{S}\left(M^{\prime}: W_{0}: h\right)^{*}$ onto $\mathscr{S}\left(M^{\prime}: W_{0}: b\right)^{*}$. Further, for any $\delta \in \mathscr{F}_{I}^{*}$ and $w^{*} \in W_{0}^{*}, L_{h}^{\prime}\left(R_{h}\left(\delta(h) \otimes w^{*}\right)\right)=R_{b}\left(\delta(b) \otimes w^{*}\right)$.

Proof. We use the notation of Lemma 5.29. Now since

$$
\tau_{1, h}^{*}\left(k_{1}\right) w^{*} \tau_{2, h}^{*}\left(k_{2}\right)=e^{b-h}\left(k_{1} k_{2}\right) \tau_{1, b}^{*}\left(k_{1}\right) w^{*} \tau_{2, b}^{*}\left(k_{2}\right),
$$

we have using Lemma 5.28(ii) that $L_{h} \otimes 1: U(h)^{*}=\mathscr{V}(h)^{*} \otimes W_{0}^{*} \rightarrow U(b)^{*}=$ $\mathscr{V}(b)^{*} \otimes W_{0}^{*}$ intertwines the representations $\left(\rho_{1}(h), \rho_{2}(h)\right)$ and $\left(\rho_{1}(b), \rho_{2}(b)\right)$. Thus, by restriction, $L_{h} \otimes 1$ gives an isomorphism of $\left(U(h)^{*}\right)^{K_{M^{\prime}}}$ onto 
$\left(U(b)^{*}\right)^{K_{M^{\prime}}}$ and an isomorphism of $\operatorname{Ker} R_{h}$ onto $\operatorname{Ker} R_{b}$. Now if $\delta \in \mathscr{F}_{I}^{*}$, $w^{*} \in W_{0}^{*}$,

$$
L_{h}^{\prime}\left(R_{h}\left(\delta(h) \otimes w^{*}\right)\right)=R_{b} \circ\left(L_{h} \otimes 1\right) \circ R_{h}^{-1} \circ R_{h}\left(\delta(h) \otimes w^{*}\right) .
$$

But $R_{h}^{-1} \circ R_{h}\left(\delta(h) \otimes w^{*}\right)=\delta(h) \otimes w^{*}+u_{h}^{*}$, where $u_{h}^{*} \in \operatorname{Ker} R_{h}$, so

$$
\left(L_{h} \otimes 1\right) \circ R_{h}^{-1} \circ R_{h}\left(\delta(h) \otimes w^{*}\right)=\delta(b) \otimes w^{*}+\left(L_{h} \otimes 1\right)\left(u_{h}^{*}\right)
$$

by Lemma $5.28(i)$. Now

$$
L_{h}^{\prime}\left(R_{h}\left(\delta(h) \otimes w^{*}\right)\right)=R_{b}\left(\delta(b) \otimes w^{*}\right)
$$

since $\left(L_{h} \otimes 1\right)\left(u_{h}^{*}\right) \in \operatorname{Ker} R_{b}$. Q.E.D.

Theorem 5.32. There is a finite open cover $\mathscr{J}_{I}$ of $\mathscr{O}_{I}$ satisfying the following. For each $J \in \mathscr{J}_{I}$ there are $\varphi_{p}^{\prime} \in \mathscr{F}_{I}\left(M^{\prime}: \tau_{1}: \tau_{2}\right), S_{p} \in \operatorname{End}_{K_{M^{\prime}}}\left(W_{0}\right), \delta_{p q} \in \mathscr{F}_{I}^{*}$, and $w_{p q}^{*} \in W_{0}^{*}, 1 \leq p \leq s, 1 \leq q \leq t_{p}$, so that for all $h \in J$

(i) $\left\{S_{p} F\left(\varphi_{p}^{\prime}: h\right)\right\}_{i=1}^{s}$ is a basis for $\mathscr{S}\left(M^{\prime}: W_{0}: h\right)$;

(ii) $\left\{R_{h}\left(\sum_{q} \delta_{p q}(h) \otimes w_{p q}^{*}\right)\right\}_{p=1}^{s}$ is a basis for $\mathscr{S}\left(M^{\prime}: W_{0}: h\right)^{*}$.

Proof. Fix $h^{\prime} \in \Omega_{I}$. By Lemma 5.19 there are a neighborhood $J^{\prime}$ of $h^{\prime}$ in $\mathscr{O}_{I}$ and $\varphi_{p}^{\prime} \in \mathscr{F}_{I}\left(M^{\prime}: \tau_{1}: \tau_{2}\right), S_{p} \in \operatorname{End}_{K_{M^{\prime}}}\left(W_{0}\right)$, so that $\left\{F_{p}(h)=S_{p} F\left(\varphi_{p}^{\prime}: h\right)\right\}_{p=1}^{s}$ is a basis for $\mathscr{S}(h)=\mathscr{S}\left(M^{\prime}: W_{0}: h\right)$ for all $h \in J^{\prime}$. Pick $\delta_{p q} \in \mathscr{F}_{I}^{*}$, $w_{p q}^{*} \in W_{0}^{*}$ so that $\left\{u_{p}^{*}\left(h^{\prime}\right)=R_{h^{\prime}}\left(\sum_{q} \delta_{p q}\left(h^{\prime}\right) \otimes w_{p q}^{*}\right)\right\}_{p=1}^{s}$ is a basis for $\mathscr{S}\left(h^{\prime}\right)^{*}=$ $\mathscr{S}\left(M^{\prime}: W_{0}: h^{\prime}\right)^{*}$. For $h \in J$, let $M(h)$ be the $s \times s$ matrix with entries $m_{p q}(h)=\left\langle u_{p}^{*}(h), F_{q}(h)\right\rangle \in \mathbf{C}$. It is proved in (8.4) that $h \mapsto \operatorname{det} M(h)$ is a smooth function of $h \in \mathscr{O}_{I}$. Now $\left\{u_{p}^{*}(h)\right\}_{p=1}^{s}$ will be a basis for $\mathscr{S}(h)^{*}$ for all $h \in J^{\prime}$ such that $\operatorname{det} M(h) \neq 0$. Since $\operatorname{det} M\left(h^{\prime}\right) \neq 0$, there is a neighborhood $J \subseteq J^{\prime}$ of $h^{\prime}$ so that $\operatorname{det} M(h) \neq 0$ for all $h \in J$. (In fact, we can assume that $\operatorname{det} M(h)$ is bounded away from zero in $J$.) Now for $h \in J,\left\{F_{p}(h)\right\}_{p=1}^{s}$ is a basis for $\mathscr{S}(h)$ and $\left\{u_{p}^{*}(h)\right\}_{p=1}^{s}$ is a basis for $\mathscr{S}(h)^{*}$. Now since $\Omega_{I}$ is compact, we can find a finite open cover $\widetilde{J_{I}}$ of $\Omega_{I}$ so that for each $\widetilde{J} \in \widetilde{\mathscr{J}_{I}}$ we have consistent bases for both $\mathscr{S}(h)$ and $\mathscr{S}(h)^{*}, h \in \widetilde{J}$. Let $\mathscr{J}_{I}=\left\{\alpha_{I}^{-1}(\widetilde{J})\right.$ : $\left.\widetilde{J} \in \widetilde{\mathscr{J}_{I}}\right\}$. As in Lemma 5.26, for $h \in \alpha_{I}^{-1}(b), b \in \widetilde{J}$, if $\left\{F_{p}(b)\right\}$ is a basis for $\mathscr{S}(b)$, then $\left\{\left(T_{h}^{\prime}\right)^{-1}\left(F_{p}(b)\right)=F_{p}(h)\right\}$ is a basis for $\mathscr{S}(h)$. Further, by Lemma 5.31, if $\left\{u_{p}^{*}(b)\right\}$ is a basis for $\mathscr{S}(b)^{*}$, then $\left\{\left(L_{h}^{\prime}\right)^{-1}\left(u_{p}^{*}(b)\right)=u_{p}^{*}(h)\right\}$ is a basis for $\mathscr{S}(h)^{*}$. Thus consistent bases in $\widetilde{J}$ give consistent bases in $\alpha_{1}^{-1}(\widetilde{J})$. Q.E.D.

Now for each $\varphi^{\prime} \in \mathscr{F}_{I}\left(M^{\prime}: \tau_{1}: \tau_{2}\right)$, by $(5.21)$ there are $\varphi_{q}^{\prime} \in \mathscr{F}\left(M^{\prime}: \tau_{1}: \tau_{2}\right)$ and rational functions $r_{q}$ with no poles in $\operatorname{cl}\left(\mathscr{O}_{I}\right)$ so that $\varphi^{\prime}(h)=\sum_{q} r_{q}(h) \varphi_{q}^{\prime}(h)$ for all $h \in \operatorname{cl}\left(\mathscr{O}_{I}\right)$. Thus by (5.12), $F\left(\varphi^{\prime}: h\right)=\sum_{q} r_{q}(h) F\left(\varphi_{q}^{\prime}: h\right)$ for all $h \in \operatorname{cl}\left(\mathscr{O}_{I}\right)$. Thus we can express the families used to form consistent bases 
for $\mathscr{S}\left(M^{\prime}: w_{0}: h\right)$ in Theorem 5.32 in terms of families $S F\left(\varphi^{\prime}: h\right), S \in$ End $_{K_{M^{\prime}}}\left(W_{0}\right), \varphi^{\prime} \in \mathscr{F}\left(M^{\prime}: \tau_{1}: \tau_{2}\right)$. Finally, in order to relate these families to the families $F \in \mathscr{S}\left(M^{\dagger}: W\right)$, we need the following.

Proposition 5.33. Let $\varphi^{\prime} \in \mathscr{F}\left(M^{\prime}: \tau_{1}: \tau_{2}\right), S \in \operatorname{End}_{K_{M^{\prime}}}\left(W_{0}\right)$. Then there is $F \in \mathscr{S}\left(M^{\dagger}: W\right)$ so that $\left.F(h)\right|_{M^{0}} \circ p=S F\left(\varphi^{\prime}: h_{M}(h)\right)$ for all $h \in \operatorname{cl}(\mathscr{D})$.

Proof. Write $I(h): \mathscr{S}\left(M^{\dagger}: W: h\right) \rightarrow \mathscr{S}\left(M^{\prime}: W_{0}: h_{M}(h)\right)$ for the isomorphism given in Lemmas 5.9 and 5.10 so that $I(h) F=\left.F\right|_{M^{0} \circ p}$ for all $F \in \mathscr{S}\left(M^{\dagger}: W: h\right)$. For all $h \in v_{\mathrm{C}}^{*}, z m^{0} \in Z_{M}\left(M^{0}\right) \cdot M^{0}=M^{\dagger}$, we can define $F\left(h: z m^{0}\right)={ }^{\prime} \tau_{1, h}(z) S F\left(\varphi^{\prime}: h_{M}(h): m^{\prime}\right)$, where $p\left(m^{\prime}\right)=m^{0}$. By Lemma 5.13, $F\left(\varphi^{\prime}\right) \in C^{\infty}\left(\left(\mathfrak{v}_{M^{\prime}}\right)_{\mathrm{C}}^{*} \times M^{\prime}\right)$ and is holomorphic as a function of $h \in\left(\mathfrak{v}_{M^{\prime}}\right)_{\mathbf{C}}^{*}$. For $h \in\left(\mathfrak{v}_{M}\right)_{\mathbf{C}}^{*}, F\left(\varphi^{\prime}: h\right)$ factors through $M^{\prime} / \operatorname{Ker} p=M^{0}$ to give a smooth map from $\left(\mathfrak{v}_{M}\right)_{\mathrm{C}}^{*} \times M^{0} \rightarrow W_{0}$ which is holomorphic in $h$. Thus $F \in C^{\infty}\left(\mathfrak{v}_{\mathrm{C}}^{*} \times M^{\dagger}: W\right)$ and is holomorphic on $\mathfrak{v}_{\mathrm{C}}^{*}$. Further, for $h \in \operatorname{cl}(\mathscr{D})$, $S F\left(\varphi^{\prime}: h_{M}(h)\right) \in \mathscr{S}\left(M^{\prime}: W_{0}: h_{M}(h)\right)$, so $I(h)^{-1} S F\left(\varphi^{\prime}: h_{M}(h)\right)=F(h) \epsilon$ $\mathscr{S}\left(M^{\dagger}: W: h\right)$. It remains to check that $F$ satisfies the growth estimate (iii) of (5.2).

Let $x=k_{1} a k_{2} \in M^{\dagger}$, where $k_{1}, k_{2} \in K_{M}^{\dagger}, a \in \operatorname{cl}\left(A_{M}^{+}\right)$. Then for $D_{1}, D_{2} \in$ $\mathscr{U}(m)$, there are $C>0$ and finitely many $D_{i}, D_{i}^{\prime} \in \mathscr{U}(m)$ so that

$$
\left\|F\left(h: D_{1} ; x ; D_{2}\right)\right\| \leq C\left|e^{h}\left(k_{1} k_{2}\right)\right| \sum_{i}\left\|F\left(h: D_{i} ; a ; D_{i}^{\prime}\right)\right\| .
$$

Now $\left|e^{h}\left(k_{1} k_{2}\right)\right| \leq e^{\left|h_{I}\right| \sigma_{V}(x)}$ for all $h \in \mathscr{D}_{\mathrm{C}}$. Now for $a \in \operatorname{cl}\left(A_{M}^{+}\right)$we can write

$$
\begin{aligned}
\left\|F\left(h: D_{i} ; a ; D_{i}^{\prime}\right)\right\| & =\left\|S F\left(\varphi^{\prime}: h_{M}(h): D_{i} ; a^{\prime} ; D_{i}^{\prime}\right)\right\| \\
& \leq\|S\|_{\mathrm{op}}\left\|F\left(\varphi^{\prime}: h_{M}(h): D_{i} ; a^{\prime} ; D_{i}^{\prime}\right)\right\|,
\end{aligned}
$$

where $p\left(a^{\prime}\right)=a$. Now by $[9,5.12,9.8]$ applied to $F\left(\varphi^{\prime}\right)$, there are $C, r \geq 0$ so that

$$
\left\|F\left(\varphi^{\prime}: h_{M}(h): D_{i} ; a^{\prime} ; D_{i}^{\prime}\right)\right\| \leq C\left(1+\left|h_{M}(h)\right|\right)^{r}\left(1+d^{\prime}\left(h_{M}(h)\right)^{-1}\right)^{r} \Xi_{M^{\prime}}\left(a^{\prime}\right),
$$

where $d^{\prime}$ denotes the distance from the boundary of $\mathscr{D}_{M^{\prime}}$. But $\Xi_{M^{\prime}}\left(a^{\prime}\right)=$ $\Xi_{M}(x)$ and there are constants $c_{i}$ so that $\left|h_{M}(h)\right| \leq c_{1}|h|$ and $d^{\prime}\left(h_{M}(h)\right) \geq$ $c_{2} d(h)$ for all $h \in \mathscr{D}$. Q.E.D.

\section{Growth estimates FOR $\Psi_{f}(h: \nu), f \in \mathscr{C}(G)$}

In this section we will study the spherical functions of matrix coefficients, $\Psi_{f}(h: \nu)$, corresponding to $f \in \mathscr{C}(G)$ as in (4.4). The main result of this section is the following. 
Theorem 6.1. Let $f \in \mathscr{C}(G)$. Then $(h, \nu, m) \rightarrow \Psi_{f}(h: \nu: m)$ is jointly smooth on $\operatorname{cl}(\mathscr{D}) \times \mathscr{F} \times M^{\dagger}$. For every polynomial coefficient differential operator $D$ on $i^{*} \times \mathscr{F}$ there are $q \geq 0$ and a continuous seminorm $\mu$ on $\mathscr{E}(G)$ so that

$$
\sup _{(h, \nu) \in \mathrm{cl}(\mathscr{D}) \times \mathscr{F}}\left\|\Psi_{f}(h: \nu ; D: m)\right\| \leq(1+\tilde{\sigma}(m))^{q} \Xi_{M}^{-1}(m) \mu(f)
$$

for all $m \in M^{\dagger}, f \in \mathscr{C}(G)$.

For any finite-dimensional real vector space $E$, let $D(E)$ denote the constant coefficient differential operators on $E$ and let $P(E)$ denote the polynomial coefficient differential operators on $E$.

Let $\mu_{i, h}$ denote the highest weight of $\tau_{i, h}, i=1,2$.

Lemma 6.2. Let $D \in D\left(i \mathfrak{v}^{*}\right)$. Then there exist $n \geq 0$ and $\omega \in \mathscr{Z}(\mathfrak{k})$ so that for all $m \geq 0, f \in \mathscr{C}(G), x \in G$,

$$
\begin{aligned}
F(h ; D: x)=\sum_{p=0}^{n}\left(\begin{array}{c}
n+1 \\
p
\end{array}\right)(-1)^{p+n}\left(1+\left\|\mu_{1, h}+\rho_{K}\right\|^{2}\right)^{m(p-n-1)} & \\
& \times F\left(h ; D: \omega^{m(n+1-p)} ; x\right) \\
=\sum_{p=0}^{n}\left(\begin{array}{c}
n+1 \\
p
\end{array}\right)(-1)^{p+n}\left(1+\left\|\mu_{2, h}+\rho_{K}\right\|^{2}\right)^{m(p-n-1)} & \\
& \times F\left(h ; D: x ; \omega^{m(n+1-p)}\right) .
\end{aligned}
$$

Proof. Only the first formula will be proved. Since

$$
F(h: k x)={ }^{\prime} \tau_{1, h}(k) F(h: x)
$$

for all $k \in K$, for all $z \in \mathscr{Z}(\mathfrak{k}), F(h: z ; x)=\chi(h: z) F(h: x)$, where $\chi(h: z)$ is a smooth function of $h$. Now if $D$ has total degree $n$,

$$
F\left(h ; D:(z-\chi(h: z))^{n+1} ; x\right)=0 .
$$

Now pick $\omega=1+\left\|\rho_{K}\right\|^{2}+\Omega_{K}$, where $\Omega_{K}$ is the Casimir element in $\mathscr{Z}(\mathfrak{k})$. Then $\chi(h: \omega)=1+\left\|\mu_{1, h}+\rho_{K}\right\|^{2}$. For any $m \geq 0$ take $z=\omega^{m}$ to obtain

$$
\sum_{p=0}^{n+1}\left(\begin{array}{c}
n+1 \\
p
\end{array}\right)(-1)^{p}\left(1+\left\|\mu_{1, h}+\rho_{K}\right\|^{2}\right)^{m p} F\left(h ; D: \omega^{m(n+1-p)} ; x\right)=0 .
$$

Now solve for $F(h ; D: x)$ which occurs in the term corresponding to $p=$ $n+1$. Q.E.D.

Lemma 6.3. Pick $t_{0}$ so that $\int_{Z}\left(1+\sigma_{V}(z)\right)^{-t_{0}} d z<\infty$. Then for any $t \geq 0$ there is a constant $C$ so that

$$
\int_{Z}\left(1+\sigma_{V}(z)\right)^{t}\left(1+\sigma_{V}(x z)\right)^{-t-t_{0}} d z \leq C\left(1+\sigma_{V}(x)\right)^{t} \text { for all } x \in G .
$$

Proof. Let $\omega_{V}$ be a compact subset of $V$ so that $\omega_{V}$ maps onto $V / Z$ under the canonical projection. Fix $x \in G$ and write $x=v y$, where $v \in V$ and 
$y \in K_{1} \exp \mathfrak{p}$. Then $\sigma_{V}(x z)=\sigma_{V}(v z)$. Pick $z_{1} \in Z$ so that $v z_{1} \in \omega_{V}$. Changing variables, $z \rightarrow z z_{1}$,

$$
\begin{aligned}
\int_{Z}(1 & \left.+\sigma_{V}(z)\right)^{t}\left(1+\sigma_{V}(x z)\right)^{-t-t_{0}} d z \\
& =\int_{Z}\left(1+\sigma_{V}\left(z z_{1}\right)\right)^{t}\left(1+\sigma_{V}\left(v z_{1} z\right)\right)^{-t-t_{0}} d z \\
& \leq\left(1+\sigma_{V}\left(z_{1}\right)\right)^{t}\left(1+\sigma_{V}\left(v z_{1}\right)\right)^{t+t_{0}} \int_{Z}\left(1+\sigma_{V}(z)\right)^{-t_{0}} d z
\end{aligned}
$$

since

$$
\left(1+\sigma_{V}\left(z z_{1}\right)\right)^{t} \leq\left(1+\sigma_{V}\left(z_{1}\right)\right)^{t}\left(1+\sigma_{V}(z)\right)^{t}
$$

and

$$
\left(1+\sigma_{V}\left(v z_{1} z\right)\right)^{-t-t_{0}} \leq\left(1+\sigma_{V}\left(v z_{1}\right)\right)^{t+t_{0}}\left(1+\sigma_{V}(z)\right)^{-t-t_{0}} .
$$

Now

$$
\begin{aligned}
(1+ & \left.\sigma_{V}\left(z_{1}\right)\right)^{t}\left(1+\sigma_{V}\left(v z_{1}\right)\right)^{t+t_{0}} \\
& \leq\left(1+\sigma_{V}\left(v z_{1}\right)\right)^{t}\left(1+\sigma_{V}\left(v^{-1}\right)\right)^{t}\left(1+\sigma_{V}\left(v z_{1}\right)\right)^{t+t_{0}} \\
& \leq C\left(1+\sigma_{V}\left(v^{-1}\right)\right)^{t}=C\left(1+\sigma_{V}(x)\right)
\end{aligned}
$$

since $v z_{1} \in \omega_{V}$. Q.E.D.

For $r \geq 0$ and a finite subset $S$ of $\mathscr{U}(\mathfrak{g}) \oplus \mathscr{U}(\mathfrak{g})$ write

$$
\mu_{r, S}(f)=\sum_{\left(b, b^{\prime}\right) \in S}\|f\|_{r, b^{\prime}}, \quad f \in \mathscr{C}(G) .
$$

Lemma 6.4. Let $D \in D\left(i \mathfrak{v}^{*}\right)$. Let $b_{1}, b_{2} \in \mathscr{U}(\mathfrak{g})$. Then there exist $t \geq 0$ and $a$ finite subset $S$ of $\mathscr{U}(\mathfrak{g}) \oplus \mathscr{U}(\mathfrak{g})$ so that for all $s \geq 0, f \in \mathscr{C}(G), x \in G$,

$$
\sup _{h \in i^{*}}\left\|F\left(h ; D: b_{1} ; x ; b_{2}\right)\right\| \leq \Xi(x)(1+\sigma(x))^{-s}\left(1+\sigma_{V}(x)\right)^{t} \mu_{t+t_{0}+s, s}(f),
$$

where $t_{0}$ is as in Lemma 6.3.

Proof. For $k_{1}, k_{2} \in K_{1}$, by (4.2),

$$
\begin{aligned}
F(h & : x)\left(k_{1}: k_{2}\right)=f\left(h: k_{1}^{-1} x k_{2}^{-1}\right) \\
& =\int_{K / Z} \int_{K} \delta\left(\tau_{1, h}^{*}: y_{1}\right) \delta\left(\tau_{2, h}: y_{2}\right) f\left(y_{1}^{-1} k_{1}^{-1} x k_{2}^{-1} y_{2}\right) d y_{1} d\left(y_{2} Z\right) \\
& =\int_{K / Z} \int_{K / Z} \delta\left(\tau_{1, h}^{*}: k_{1}^{-1} y_{1}\right) \delta\left(\tau_{2, h}: k_{2} y_{2}\right) g\left(h: y_{1}^{-1} x y_{2}\right) d\left(y_{1} Z\right) d\left(y_{2} Z\right),
\end{aligned}
$$

where $g(h: x)=\int_{Z} f(x z) \zeta(h: z) d z$. Here $\zeta(h)=\zeta(0) \otimes e^{h}$ is the $Z$ character of both $\tau_{1, h}$ and $\tau_{2, h}$. Thus for $D, b_{1}$, and $b_{2}$ as in the lemma, 
there are finitely many $D_{i}^{\prime}, D_{i}^{\prime \prime}, b_{i}^{\prime}, b_{i}^{\prime \prime}$ so that

$$
\begin{gathered}
\left\|F\left(h ; D: b_{1} x ; b_{2}\right)\right\|=\sup _{k_{1}, k_{2} \in K_{1}}\left|F\left(h ; D: b_{1} ; x ; b_{2}\right)\left(k_{1}: k_{2}\right)\right| \\
\leq \sup _{k_{1}, k_{2}} \sum_{i} \int_{\omega_{K} \times \omega_{K}} \mid D_{i}^{\prime}\left(\delta\left(\tau_{1, h}^{*}: k_{1}^{-1} y_{1}\right) \delta\left(\tau_{2, h}: k_{2} y_{2}\right)\right) \\
a_{i}\left(y_{1}\right) a_{i}^{\prime}\left(y_{2}\right) g\left(h ; D_{i}^{\prime \prime}: b_{i}^{\prime} ; y_{1}^{-1} x y_{2} ; b_{i}^{\prime \prime}\right) \mid d y_{1} d y_{2},
\end{gathered}
$$

where the $a_{i}, a_{i}^{\prime}$ are smooth functions on $K / Z$ and $\omega_{K}=K_{1} \times \omega_{V}$, where $\omega_{V}$ is a compact subset of $V$ which maps onto $V / Z$ under the canonical projection. Now

$$
\max _{i} \sup _{h} \sup _{\substack{y_{1}, y_{2} \in \omega_{K} \\ k_{1}, k_{2} \in K_{1}}}\left|a_{i}\left(y_{1}\right) a_{i}^{\prime}\left(y_{2}\right) D_{i}\left(\delta\left(\tau_{1, h}^{*}: k_{1}^{-1} y_{1}\right) \delta\left(\tau_{2, h}: k_{2} y_{2}\right)\right)\right|=C<\infty .
$$

Thus,

$$
\left\|F\left(h ; D: b_{1} ; x ; b_{2}\right)\right\| \leq C\left(\operatorname{Vol} \omega_{K}\right)^{2} \sum_{i} \sup _{y_{1}, y_{2} \in \omega_{K}}\left|g\left(h ; D_{i}^{\prime \prime}: b_{i}^{\prime} ; y_{1}^{-1} x y_{2} ; b_{i}^{\prime \prime}\right)\right| \text {. }
$$

But for all $x$,

$$
\left|g\left(h ; D_{i}^{\prime \prime}: b_{i}^{\prime} ; x ; b_{i}^{\prime \prime}\right)\right| \leq \int_{Z}\left|\zeta\left(h ; D_{i}^{\prime \prime}: z\right)\right|\left|f\left(b_{i}^{\prime} ; x z ; b_{i}^{\prime \prime}\right)\right| d z .
$$

Now there are $t$ and $C$ depending on $D$ so that $\left|\zeta\left(h ; D_{i}^{\prime \prime}: z\right)\right| \leq C\left(1+\sigma_{V}(z)\right)^{t}$ for all $i$. Then for all $s \geq 0$,

$$
\begin{aligned}
& \left|g\left(h ; D_{i}^{\prime \prime}: b_{i c}^{\prime} x ; b_{i}^{\prime \prime}\right)\right| \\
& \quad \leq C_{1} \Xi(x)_{b_{i}^{\prime}}\|f\|_{s+t+t_{0}, b_{i}^{\prime \prime}} \int_{Z}\left(1+\sigma_{V}(z)\right)^{t}(1+\tilde{\sigma}(x z))^{-t-t_{0}-s} d z .
\end{aligned}
$$

But $(1+\tilde{\sigma}(x z))^{-t-t_{0}-s} \leq C^{\prime}(1+\sigma(x))^{-s}\left(1+\sigma_{V}(x z)\right)^{-t-t_{0}}$ since $\tilde{\sigma}(x z) \geq$ $\sigma(x z)=\sigma(x)$ and $\tilde{\sigma}(x z) \geq \sigma_{V}(x z)$ for all $z$. Now by Lemma 6.3,

$$
\left|g\left(h ; D_{i}^{\prime \prime}:^{\prime} b_{i} ; x ; b_{i}^{\prime \prime}\right)\right| \leq C \Xi(x)_{b_{i}^{\prime}}\|f\|_{s+t+t_{0}, b_{i}^{\prime \prime}}(1+\sigma(x))^{-s}\left(1+\sigma_{V}(x)\right)^{t} .
$$

But now, for all $y_{1}, y_{2} \in \omega_{K}, \Xi\left(y_{1}^{-1} x y_{2}\right)=\Xi(x)$ and $\sigma\left(y_{1}^{-1} x y_{2}\right)=\sigma(x)$. Further, since $\omega_{K}$ is compact there is a constant $c$ so that $\sup _{y_{1}, y_{2} \in \omega_{K}} \sigma_{V}\left(y_{1}^{-1} x y_{2}\right)$ $\leq c+\sigma_{V}(x)$. Thus there is a constant $C_{0}$ so that

$$
\begin{aligned}
& \left\|F\left(h ; D: b_{1} ; x ; b_{2}\right)\right\| \\
& \quad \leq C_{0} \Xi(x)(1+\sigma(x))^{-s}\left(1+\sigma_{V}(x)\right)^{t} \sum_{i} b_{i}^{\prime}\|f\|_{s+t+t_{0}, b_{i}^{\prime \prime}} \quad \text { Q.E.D. }
\end{aligned}
$$

Fix a $\theta$-stable Cartan subgroup $H=T \times A$ of $G$ and a parabolic subgroup $P=M A N$ corresponding to $H$. For $f \in \mathscr{C}(G)$, define $G_{f}$ as in (4.3a). 
Theorem 6.5. Let $f \in \mathscr{C}(G)$. Then $(h, \nu, m) \rightarrow G_{f}(h: \nu: m)$ is jointly smooth on $i v^{*} \times \mathscr{F} \times M^{\dagger}$. Let $D \in P\left(i v^{*} \times \mathscr{F}\right)$ and let $r \geq 0$. Then there are $t \geq 0$ and a continuous seminorm $\mu$ on $\mathscr{C}(G)$ so that for all $m \in M^{\dagger}$, $f \in \mathscr{C}(G)$,

$$
\sup _{(h, \nu) \in i 0^{*} \times F^{-}}\left\|G_{f}(h: \nu ; D: m)\right\| \leq(1+\sigma(m))^{-r}\left(1+\sigma_{V}(m)\right)^{t} \Xi_{M}(m) \mu(f) .
$$

Proof. Write $G_{f}(h: m a)=e^{\rho_{p}}(a) \int_{N} F(h:$ man $) d n$ so that $G_{f}(h: \nu: m)=$ $\int_{A} e^{-i \nu}(a) G_{f}(h: m a) d a$. We know from [6, 5.3] that $a \mapsto G_{f}(h: m a)$ is a Schwartz function on $A$ for all $h, m$. There are $q \geq 0$ and finitely many $D_{i}^{\prime} \in D\left(i v^{*}\right), D_{i}^{\prime \prime} \in D(\mathscr{F})$ so that

$\left\|G_{f}(h: \nu ; D: m)\right\| \leq(1+|h|)^{q}(1+|\nu|)^{q} \sum_{i}\left\|D_{i}^{\prime \prime} \int_{A} e^{-i \nu}(a) G_{f}\left(h ; D_{i}^{\prime}: m a\right) d a\right\|$.

By abelian Fourier analysis on $A$ there are $s_{i}>0$ and $u_{i} \in \mathscr{S}(\mathfrak{a})$ depending on $q, D_{i}^{\prime \prime}$, so that for all $h, m$,

$$
\begin{aligned}
\sup _{\nu \in \mathscr{F}}(1+|\nu|)^{q}\left\|D_{i}^{\prime \prime} \int_{A} e^{-i \nu}(a) G_{f}\left(h ; D_{i}^{\prime}: m a\right) d a\right\| \\
\quad \leq \sup _{a \in A}(1+\sigma(a))^{s_{i}}\left\|G_{f}\left(h ; D_{i}^{\prime}: u_{i} i m a\right)\right\| \\
\quad \leq \sup _{a \in A}(1+\sigma(a))^{s} \sum_{j}\left|e^{\rho_{p}}\left(a ; u_{j}^{\prime}\right)\right| \int_{N}\left\|F\left(h ; D_{i}^{\prime}: u_{j}^{\prime \prime} ; m a n\right)\right\| d n,
\end{aligned}
$$

where $s=\max \left\{s_{i}\right\}$ and $u_{j}^{\prime}, u_{j}^{\prime \prime} \in \mathscr{S}(\mathfrak{a})$. Now $e^{\rho_{p}}\left(a ; u_{j}^{\prime}\right)=\rho_{p}\left(u_{j}^{\prime}\right) e^{\rho_{p}}(a)$, so we have

$$
\begin{aligned}
& \quad \sup _{(h, \nu) \in i 0^{*} \times \mathscr{F}^{-}}\left\|G_{f}(h: \nu ; D: m)\right\| \\
& \quad \leq \sup _{\substack{a \in A \\
h \in i 0^{*}}}(1+|h|)^{q}(1+\sigma(a))^{s} \sum_{i, j}\left|\rho_{p}\left(u_{j}^{\prime}\right)\right| e^{\rho_{p}(a)} \int_{N}\left\|F\left(h ; D_{i}^{\prime}: u_{j}^{\prime \prime} ; m a n\right)\right\| d n .
\end{aligned}
$$

Pick $m$ large enough that $\sup _{h \in i 0^{*}}(1+|h|)^{q}\left(1+\left\|\mu_{2, h}+\rho_{k}\right\|^{2}\right)^{-m}<\infty$ and pick $\omega \in \mathscr{Z}(\mathfrak{k})$ as in (6.2). Then for each $i$ there is $n_{i} \geq 0$ so that

$$
\begin{gathered}
(1+|h|)^{q}\left\|F\left(h ; D_{i}^{\prime}: u_{j}^{\prime \prime} ; \operatorname{man}\right)\right\| \\
\leq(1+|h|)^{q} \sum_{p=0}^{n_{i}}\left(\begin{array}{c}
n_{i}+1 \\
p
\end{array}\right)\left(1+\left\|\mu_{2, h}+\rho_{K}\right\|^{2}\right)^{m\left(p-n_{i}-1\right)} \\
\quad \times\left\|F\left(h ; D_{i}^{\prime}: u_{j}^{\prime \prime} ; \operatorname{man} ; \omega^{m\left(n_{i}+1-p\right)}\right)\right\| \\
\leq C \sum_{p=0}^{n_{i}}\left\|F\left(h ; D_{i}^{\prime}: u_{j}^{\prime \prime} ; \operatorname{man} ; \omega^{m\left(n_{i}+1-p\right)}\right)\right\| .
\end{gathered}
$$


But using Lemma 6.4 there are $t \geq 0$ and a finite subset $S$ of $\mathscr{U}(\mathfrak{g}) \oplus \mathscr{U}(\mathfrak{g})$ so that for all $i, j, r \geq 0,0 \leq p \leq n_{i}$,

$$
\begin{aligned}
\sup _{h \in i \mathfrak{v}^{*}} & \left\|F\left(h ; D_{i}^{\prime}: u_{j}^{\prime \prime} ; \operatorname{man} ; \omega^{m\left(n_{i}+1-p\right)}\right)\right\| \\
\leq & \Xi(\operatorname{man})(1+\sigma(\operatorname{man}))^{-r}\left(1+\sigma_{V}(\text { man })\right)^{t} \mu_{t+t_{0}+r, S}(f) .
\end{aligned}
$$

Thus there are $s, t \geq 0, S \subseteq \mathscr{U}(\mathfrak{g}) \oplus \mathscr{U}(\mathfrak{g})$ such that for all $r_{1} \geq 0$

$$
\begin{aligned}
\sup _{(h, \nu) \in i \mathrm{v}^{*} \times \mathscr{F}} & \left\|G_{f}(h: \nu ; D: m)\right\| \\
\leq C & \mu_{t+t_{0}+r_{1}, S}(f) \sup _{a \in A}(1+\sigma(a))^{s} e^{\rho_{p}}(a) \\
& \quad \times \int_{N} \Xi(\operatorname{man})(1+\sigma(\text { man }))^{-r_{1}}\left(1+\sigma_{V}(\text { man })\right)^{t} d n .
\end{aligned}
$$

Now using [9, 2.12] $\sigma_{V}($ man $) \leq C+\sigma_{V}(m a)+\sigma_{V}(n) \leq C_{1}+C_{2} \sigma_{V}(m)$ for all $a, n$ since $\sigma_{V}(m a)=\sigma_{V}(m)$ and $\sup _{n \in N} \sigma_{V}(n)<\infty$ by [9, 2.13]. Further, by $[6,5.2]$, there is $d \geq 0$ so that for any $r \geq 0$,

$$
e^{\rho_{p}}(a) \int_{N} \Xi(\operatorname{man})(1+\sigma(\text { man }))^{-r_{1}} d n \leq C \Xi_{M}(m)(1+\sigma(m))^{-r}(1+\sigma(a))^{-s}
$$

for all $r_{1}>r+s+2 d$. Thus for $r_{1}>r+s+2 d$,

$$
\begin{aligned}
& \sup _{(h, \nu) \in i 0^{*} \times \mathscr{F}}\left\|G_{f}(h: \nu ; D: m)\right\| \\
& \quad \leq C \mu_{t+t_{0}+r_{1}, S}(f) \Xi_{M}(m)(1+\sigma(m))^{-r}\left(1+\sigma_{V}(m)\right)^{t} \text {. Q.E.D. }
\end{aligned}
$$

Lemma 6.6 (J. A. Wolf). Suppose $h \in G$ belongs to a $\theta$-stable Cartan subgroup of $G$. Then $\sigma(h) \leq \sigma\left(y h y^{-1}\right)$ for all $y \in G$.

Proof. Let $X=G / K$. Then [13, Lemma 3.6] shows that $h$ stabilizes a geodesic of $X$ which passes through the point $x_{0}=1 \cdot K \in G / K$. The displacement function $\delta: X \rightarrow \mathbf{R}$ given by $\delta(x)=\operatorname{distance}(x, h x)$ achieves its minimum on the union of the $h$-invariant geodesics of $X$. (See the construction of the flat strip between invariant geodesics in [14, Theorem 1].) But $\sigma(h)=$ $\delta\left(x_{0}\right)$ and $\sigma\left(y h y^{-1}\right)=\operatorname{distance}\left(x_{0}, y h y^{-1} x_{0}\right)=\operatorname{distance}\left(y^{-1} \ddot{x}_{0}, h y^{-1} x_{0}\right)=$ $\delta\left(y^{-1} x_{0}\right)$. Q.E.D.

Lemma 6.7 (J. A. Wolf). There is a constant $C^{\prime}>0$ so that

$$
\sigma_{V}\left(\exp \xi_{0} \exp \xi_{1} \cdots \exp \xi_{n}\right) \leq n C^{\prime} \text { for all } n \geq 1, \xi_{0}, \ldots, \xi_{n} \in \mathfrak{p} .
$$

Proof. Suppose first that $G$ is simply connected. Then it is sufficient to prove the result when $G$ is a vector group or is simple. If $G$ is a vector group, then $\mathfrak{p}=\{0\}$ so the result is trivial. If $G$ is simple, then $\sigma_{V}=0$ unless $G$ is noncompact of hermitian type. For such $G$, the assertion is proved with $n=1$ in [8, 10.6 and 10.7]. The general case is proved by induction on $n$. Assume it is true for some $n \geq 1$. Then if $\xi_{0}, \ldots, \xi_{n}, \xi_{n+1} \in \mathfrak{p}$ we can decompose $\exp \xi_{0} \cdots \exp \xi_{n}=v k \exp \eta \in V \cdot K_{1} \cdot \exp \mathfrak{p}$ and $\|v\| \leq n C^{\prime}$ by 
the induction hypothesis. Now $\exp \xi_{0} \cdots \exp \xi_{n} \exp \xi_{n+1}=v k v^{\prime} k^{\prime} \exp \eta^{\prime}$, where $\exp \eta \exp \xi_{n+1}=v^{\prime} k^{\prime} \exp \eta^{\prime} \in V \cdot K_{1} \cdot \exp \mathfrak{p}$. By the case $n=1,\left\|v^{\prime}\right\| \leq C^{\prime}$. Thus, $\sigma_{V}\left(\exp \xi_{0} \cdots \exp \xi_{n+1}\right)=\left\|v v^{\prime}\right\| \leq\|v\|+\left\|v^{\prime}\right\| \leq(n+1) C^{\prime}$.

Now for general (connected) $G$, let $p: G^{\prime} \rightarrow G$ denote the universal covering group, $K^{\prime}=p^{-1}(K)$. Then as in $[9,2.1], K^{\prime}=\left[K^{\prime}, K^{\prime}\right] \times U^{\prime} \times V^{\prime}$, where $\left[K^{\prime}, K^{\prime}\right]$ is compact, $U^{\prime}$ and $V^{\prime}$ are vector groups, $p$ maps $V^{\prime}$ isomorphically onto $V$, and $K_{1}=p\left(\left[K^{\prime}, K^{\prime}\right] \times U^{\prime}\right)$ is compact. Since $U^{\prime} \times V^{\prime}$ is a direct product, there is a constant $C$ so that for all $u^{\prime} \in U^{\prime}$ and $v^{\prime} \in V^{\prime},\left\|v^{\prime}\right\| \leq$ $C\left\|u^{\prime} v^{\prime}\right\|$. Thus for all $x^{\prime} \in G^{\prime}, \sigma_{V}\left(p\left(x^{\prime}\right)\right) \leq C \sigma_{U^{\prime} \times V^{\prime}}\left(x^{\prime}\right)$. Thus the result for $G$ follows from the result for $G^{\prime}$. Q.E.D.

Lemma 6.8 (J. A. Wolf). There is a constant $C>0$ such that for all $x, y \in G$, $\sigma_{V}(x) \leq \sigma_{V}\left(y x y^{-1}\right)+C$.

Proof. By [6, 4.2b], $\sigma_{V}\left(k x k^{-1}\right)=\sigma_{V}(x)$ for all $k \in K$. Thus we may as well assume that $y=\exp \eta, \eta \in \mathfrak{p}$. Write $x=v k \exp \xi, v \in V, k \in K_{1}, \xi \in \mathfrak{p}$. Then

$$
y x y^{-1}=v k \exp \left(\operatorname{Ad}(v k)^{-1} \eta\right) \exp \xi \exp (-\eta)=v v^{\prime} k k^{\prime} \exp \eta^{\prime},
$$

where we decompose

$$
\exp \left(\operatorname{Ad}(v k)^{-1} \eta\right) \exp \xi \exp (-\eta)=v^{\prime} k^{\prime} \exp \eta^{\prime} \in V K_{1} \exp (\mathfrak{p}) .
$$

Thus $\sigma_{V}\left(y x y^{-1}\right)=\left\|v v^{\prime}\right\| \geq\|v\|-\left\|v^{\prime}\right\|$, where $\|v\|=\sigma_{V}(x)$ and $\left\|v^{\prime}\right\|=$ $\sigma_{V}\left(\exp \left(\operatorname{Ad}(v k)^{-1} \eta\right) \exp \xi \exp (-\eta)\right) \leq 2 C^{\prime}$ by Lemma 6.7. Q.E.D.

Combining Lemmas 6.6-6.8 we obtain the following.

Proposition 6.9 (J. A. Wolf). There is a constant $C>0$ so that if $h \in G$ belongs to a $\theta$-stable Cartan subgroup of $G$, then $\tilde{\sigma}(h) \leq \tilde{\sigma}(x h x)^{-1}+C$ for all $x \in G$.

Now for fixed $\lambda_{0} \in \Lambda_{M, 0}, \chi_{0} \in X\left(\lambda_{0}\right)$, and $\mathscr{D} \in \mathscr{C}\left(\lambda_{0}\right)$, we define $\Theta\left(M^{\dagger}: h\right), h \in \operatorname{cl}(\mathscr{D})$, as in (4.4), to be the characters of the corresponding family of relative discrete series representations of $M^{\dagger}$.

Lemma 6.10. Let $D \in D\left(i \mathfrak{v}^{*}\right)$. Then there are $C, s \geq 0$ so that for all $y \in M^{\dagger}$,

$$
\sup _{h \in \mathrm{cl}(\mathscr{D})}\left|\Theta\left(M^{\dagger}: h ; D: y\right)\right| \leq C\left|\Delta_{M}(y)\right|^{-1}(1+\tilde{\sigma}(y))^{s} .
$$

Here $\Delta_{M}$ denotes the Weyl denominator of the characters $\Theta\left(M^{\dagger}: h\right)$.

Proof. For $y \in M^{\dagger}$, write $y=\gamma m \times m^{-1}$, where $\gamma \in Z_{M}\left(M^{0}\right), m \in M^{0}$, and $x \in H_{M}$, an $\theta$-stable Cartan subgroup of $M^{0}$. Then, using the notation of (9.1),

$$
\Theta\left(M^{\dagger}: h: y\right)=\left(\chi_{0} \otimes e^{h}\right)(\gamma) \Delta_{M}^{-1}(x) \sum_{w \in W_{H_{M}}} c(w: \mathscr{C}: x) e^{c\left(w\left(\lambda_{0}+h_{M}(h)\right)\right)}(x) .
$$


Here $c(w: \mathscr{C}: x)=0$ unless $\left|e^{c\left(w\left(\lambda_{0}+h_{M}(h)\right)\right)}(x)\right| \leq 1$. Thus there are $C, s \geq 0$ depending on $D$ so that for all $w \in W_{H_{M}}$,

$$
\left|D\left(\left(\chi_{0} \otimes e^{h}\right)(\gamma) e^{c\left(w\left(\lambda_{0}+h_{M}(h)\right)\right)}(x)\right)\right| \leq C(1+\tilde{\sigma}(\gamma x))^{s} .
$$

The constants $c(w: \mathscr{C}: x)$ are independent of $h$ and there are only finitely many possible values as $y$ ranges over $M^{\dagger}$. Thus there is $C \geq 0$ so that $\left|\Theta\left(M^{\dagger}: h ; D: y\right)\right| \leq C\left|\Delta_{M}^{-1}(x)\right|(1+\tilde{\sigma}(\gamma x))^{s}$. But $\left|\Delta_{M}(x)\right|=\left|\Delta_{M}(y)\right|$, and by Proposition 6.9 there is a constant $C$ so that $(1+\tilde{\sigma}(\gamma x))^{s} \leq C(1+\tilde{\sigma}(y))^{s}$. Q.E.D.

Proof of Theorem 6.1. Recall that for all $f \in \mathscr{C}(G)$,

$$
\Psi_{f}(h: \nu: m)=\left[M / M^{\dagger}\right] \int_{M^{\dagger} / Z} \overline{\Theta\left(M^{\dagger}: h: y\right)} G_{f}(h: \nu: y m) d(y Z) .
$$

Now given $D \in P\left(i \mathrm{v}^{*} \times \mathscr{F}\right)$ there are finitely many $D_{i} \in P\left(i \mathrm{v}^{*} \times \mathscr{F}\right), D_{i}^{\prime} \in$ $D\left(i^{*}\right)$ so that

$$
D\left(\overline{\boldsymbol{\Theta}\left(M^{\dagger}: h: y\right)} G_{f}(h: \nu: y m)\right)=\sum_{i} \overline{\boldsymbol{\Theta}\left(M^{\dagger}: h ; D_{i}^{\prime}: y\right)} G_{f}\left(h: \nu ; D_{i}: y m\right) .
$$

Pick a fundamental domain $\omega$ for $M^{\dagger} / Z$ on which $\sigma_{V}$ is bounded. Then $\left\|\Psi_{f}(h: \nu ; D: m)\right\| \leq\left[M / M^{\dagger}\right] \sum_{i} \int_{\omega}\left|\Theta\left(M^{\dagger}: h ; D_{i}^{\prime}: y\right)\right|\left\|G_{f}\left(h: \nu ; D_{i}: y m\right)\right\| d y$

if we can justify differentiating under the integral. By Lemma 6.10 there are $C, s \geq 0$ so that for all $y \in \omega, h, i$,

$$
\left|\Theta\left(M^{\dagger}: h ; D_{i}^{\prime}: y\right)\right| \leq C\left|\Delta_{M}(y)\right|^{-1}(1+\tilde{\sigma}(y))^{s} \leq C\left|\Delta_{M}(y)\right|^{-1}(1+\sigma(y))^{s}
$$

since $\sigma_{V}$ is bounded on $\omega$. Now using Theorem 6.5 , for all $r \geq 0$ there are $t$ and $\mu_{r}$ so that for all $y \in \omega, m \in M^{\dagger}, i, h, \nu, f$,

$$
\left\|G_{f}\left(h: \nu ; D_{i}: y m\right)\right\| \leq(1+\sigma(y m))^{-r}\left(1+\sigma_{V}(y m)\right)^{t} \Xi_{M}(y m) \mu_{r}(f) .
$$

Now $(1+\sigma(y m))^{-r} \leq(1+\sigma(y))^{-r}(1+\sigma(m))^{r}$, and $\left(1+\sigma_{V}(y m)\right)^{t} \leq$ $C\left(1+\sigma_{V}(m)\right)^{t}$ since $\sigma_{V}$ is bounded on $\omega$. Further, using [8, 10.12] there are $C, d \geq 0$ so that $\Xi_{M}(y m) \leq C \Xi_{M}(y) \Xi_{M}(m)^{-1}(1+\sigma(m))^{d}$. Thus for all $r \geq 0$ there is $\mu_{r}$ so that

$$
\begin{aligned}
& \left|\Theta\left(M^{\dagger}: h ; D_{i}^{\prime}: y\right)\right|\left\|G_{f}\left(h: \nu ; D_{i}: y m\right)\right\| \\
& \quad \leq \mu_{r}(f) \Xi_{M}(y)\left|\Delta_{M}(y)\right|^{-1}(1+\sigma(y))^{s-r}(1+\sigma(m))^{r+d}\left(1+\sigma_{V}(m)\right)^{t} \Xi_{M}(m)^{-1} .
\end{aligned}
$$

But by $[6,2.15]$ there is $r \geq 0$ large enough that

$$
\int_{M^{\dagger} / Z}(1+\sigma(y))^{s-r} \Xi_{M}(y)\left|\Delta_{M}(y)\right|^{-1} d(y Z)<\infty .
$$


Thus we see that $\Psi_{f}$ is smooth and that we can differentiate inside the integral to obtain the inequality

$$
\left\|\Psi_{f}(h: \nu ; D: m)\right\| \leq C \mu_{r}(f)(1+\sigma(m))^{r+d}\left(1+\sigma_{V}(m)\right)^{t} \Xi_{M}(m)^{-1}
$$

for $r$ sufficiently large. Q.E.D.

\section{Growth estimates FOR $\Psi_{f}(h: \nu), f \in \mathscr{C}_{H}(G)$}

In $\S 6$ we obtained an estimate for $\Psi_{f}$ which is valid for any $f \in \mathscr{C}(G)$. In this section we will prove a stronger estimate when $f \in \mathscr{C}_{H}(G)=\{f \in \mathscr{C}(G)$ : $\left.f=f_{H}\right\}$. Here $f_{H}$ is defined as in (3.1b). Thus $\mathscr{C}_{H}(G)$ is the space of Schwartz class functions with Plancherel formula expansions involving only the series of tempered representations corresponding to the Cartan subgroup $H$.

For $h \in \mathscr{D} \subset i v^{*}$, define

$$
d(h) \text { : The Euclidean distance from } h \text { to the boundary of } \mathscr{D} \text {. }
$$

Theorem 7.2. For every $s \geq 0$ and every polynomial coefficient differential operator $D$ on $i v^{*} \times \mathscr{F}$, there are $t \geq 0$ and a continuous seminorm $\mu$ on $f \in \mathscr{C}(G)$ so that

$$
\sup _{(h, \nu) \in \mathscr{D} \times \mathscr{F}}\left(1+d(h)^{-1}\right)^{s}\left\|\Psi_{f}(h: \nu ; D: m)\right\| \leq(1+\tilde{\sigma}(m))^{t} \Xi_{M}(m)^{-1} \mu(f)
$$

for all $f \in \mathscr{C}_{H}(G), m \in M^{\dagger}$.

Recall for any $f \in \mathscr{C}(G)$ we have defined $G_{f}(h: \nu) \in \mathscr{C}\left(M^{\dagger} / Z, \zeta(h): W\right)$ for all $(h, \nu) \in i \mathrm{v}^{*} \times \mathscr{F}$. Let ${ }^{\circ} \mathscr{C}\left(M^{\dagger} / Z, \zeta(h): W\right)$ denote the space of cusp forms in $\mathscr{C}\left(M^{\dagger} / Z, \zeta(h): W\right)$. It is defined as follows. For any $F \in$ $\mathscr{C}\left(M^{\dagger} / Z, \zeta(h): W\right)$ and parabolic subgroup ${ }^{*} Q={ }^{*} M^{*} A^{*} N$ of $M^{\dagger}$, define

$$
F^{*} Q_{(x)}=\int_{*_{N}} F(x n) d n, \quad x \in M^{\dagger} \text {. }
$$

Then

$$
\begin{array}{r}
{ }^{\circ} \mathscr{C}\left(M^{\dagger} / Z, \zeta(h): W\right)=\left\{F \in \mathscr{C}\left(M^{\dagger} / Z, \zeta(h): W\right): F^{*} Q=0 \text { for all } x \in M^{\dagger},\right. \\
\text { for all proper parabolic subgroups } \left.{ }^{*} Q \text { of } M^{\dagger}\right\} .
\end{array}
$$

Lemma 7.4. Assume that $f \in \mathscr{C}_{H}(G)$. Then $G_{f}(h: \nu) \in{ }^{\circ} \mathscr{C}\left(M^{\dagger} / Z: \zeta(h): W\right)$ for all $(h, \nu) \in i^{*} \times \mathscr{F}$.

Proof. Let ${ }^{*} Q={ }^{*} M^{*} A^{*} N$ be a proper parabolic subgroup of $M^{\dagger}$. Then ${ }^{*} Q=$ $Q \cap M^{\dagger}$, where $Q=M_{Q} A_{Q} N_{Q}$ is a parabolic subgroup of $G$ with ${ }^{*} M=$ $M_{Q} \cap M^{\dagger}, A_{Q}={ }^{*} A \cdot A$, and $N_{Q}={ }^{*} N \cdot N$. To show $G_{f}(h: \nu)$ is a cusp form

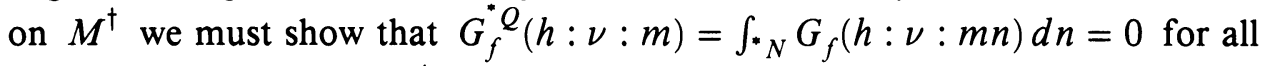
$(h, \nu, m) \in i \mathrm{v}^{*} \times \mathscr{F} \times M^{\dagger}$. 
Using [6, 5.2], $(h, \nu) \mapsto G_{f}^{*}(h: \nu: m)$ is jointly smooth on $i v^{*} \times \mathscr{F} \times M^{\dagger}$. Thus it is enough to show it is zero on the dense set of $h$ for which $F(h: x)$ factors through a group of Harish-Chandra class. But there, using [3, 27.1], $F(h)$ is a sum of wave packets coming from the $H$-series of representations, and using [3, 20.2], $F^{Q}(h: m a)=0$ for all $(m, a) \in M^{\dagger} \times A$ since $\operatorname{dim} A_{Q}>$ $\operatorname{dim} A$. Thus

$$
\begin{aligned}
G^{*}(h: \nu: m) & =\int_{*_{N}} \int_{A} e^{\left(i \nu+\rho_{p}\right)(\log a)} \int_{N} F\left(h: m a n n^{*}\right) d n d a d n^{*} \\
& =\int_{A} e^{\left(i \nu+\rho_{p}\right)(\log a)} F^{Q}(h: m a) d a=0 \text { for all }(\nu, m) \in \mathscr{F} \times M^{\dagger} .
\end{aligned}
$$

Q.E.D.

Now fix parameters $\lambda_{0} \in \Lambda_{M, 0}$ and $\chi_{0} \in X\left(\lambda_{0}\right)$ as in (3.2). For each $\mathscr{D} \in$ $\mathscr{C}\left(\lambda_{0}\right)$ define $\varepsilon(\mathscr{D})=\operatorname{sign} \prod_{\alpha \in \Phi_{M}^{+}}\left\langle\alpha, \lambda_{0}+h_{M}(h)\right\rangle$, where $h \in \mathscr{D}$. For any $h \in \operatorname{cl}(\mathscr{D})$ let $\Theta\left(M^{\dagger}: h: \mathscr{D}\right)$ denote the character of the corresponding relative discrete series representation or limit of relative discrete series representation from $\mathscr{D}$. Now for any $h \in i^{*}{ }^{*}$ we define

$$
\widetilde{\Theta}\left(M^{\dagger}: h\right)=[D(h)]^{-1} \sum_{\mathscr{D} \in D(h)} \varepsilon(\mathscr{D}) \Theta\left(M^{\dagger}: h: \mathscr{D}\right),
$$

where $D(h)=\left\{\mathscr{D} \in \mathscr{C}\left(\lambda_{0}\right): h \in \operatorname{cl}(\mathscr{D})\right\}$. For $f \in \mathscr{C}(G)$ set

$$
\begin{aligned}
\widetilde{\Psi}_{f}(h: \nu: m) & =\prod_{\alpha \in \Phi_{M}^{+}}\left\langle\alpha, \lambda_{0}+h_{M}(h)\right\rangle\left[M / M^{\dagger}\right] \widetilde{\Theta}\left(M^{\dagger}: h: R(m) G_{f}(h: \nu)\right) \\
& =[D(h)]^{-1}\left|\prod_{\alpha \in \Phi_{M}^{+}}\left\langle\alpha, \lambda_{0}+h_{M}(h)\right\rangle\right| \sum_{\mathscr{D} \in D(h)} \Psi_{f}(h: \mathscr{D}: \nu: m),
\end{aligned}
$$

where $\Psi_{f}(h: \mathscr{D}: \nu: m)$ is defined as in (4.4) from $\Theta\left(M^{\dagger}: h: \mathscr{D}\right)$. Write $\left(i \mathfrak{v}^{*}\right)^{\prime}=\left\{h \in i \mathfrak{v}^{*}: \prod_{\alpha \in \Phi_{M}^{+}}\left\langle\alpha, \lambda_{0}+h_{M}(h)\right\rangle \neq 0\right\},\left(i \mathfrak{v}^{*}\right)^{s}=\left(i \mathfrak{v}^{*}\right) \backslash\left(i \mathfrak{v}^{*}\right)^{\prime}$. Similarly, we define $\left(i \mathfrak{v}_{M}^{*}\right)^{\prime},\left(i \mathfrak{v}_{M}^{*}\right)^{s},\left(i \mathfrak{v}_{M^{\prime}}^{*}\right)^{\prime}$, and $\left(i \mathfrak{v}_{M^{\prime}}^{*}\right)^{s}$.

Lemma 7.6. Suppose $f \in \mathscr{C}_{H}(G)$. Then $\widetilde{\Psi}_{f} \in C^{\infty}\left(i \mathfrak{v}^{*} \times \mathscr{F} \times M^{\dagger}: W\right)$. Further:

(i) $\widetilde{\Psi}_{f}(h: \nu) \in \mathscr{S}\left(M^{\dagger}: W: h\right)$ for all $h \in\left(i v^{*}\right)^{\prime}, \nu \in \mathscr{F}$;

(ii) $\widetilde{\Psi}_{f}(h: \nu) \equiv 0$ for all $h \in\left(i v^{*}\right)^{s}, \nu \in \mathscr{F}$.

Proof. As in Theorem 6.5, $G_{f} \in C^{\infty}\left(i \mathrm{v}^{*} \times \mathscr{F} \times M^{\dagger}: W\right)$. Further, since each $G_{f}(h: \nu) \in{ }^{\circ} \mathscr{C}\left(M^{\dagger} / Z, \zeta(h): W\right)$ by Lemma 7.4 , we see as in $[8,10.9]$ that

$$
\widetilde{\Theta}\left(M^{\dagger}: h: R(m) G_{f}(h: \nu)\right)=\int_{M^{T} / Z} \overline{\widetilde{\Theta}\left(M^{\dagger}: h: y\right)} G_{f}(h: \nu: y m) d(y Z),
$$

where $T$ is a relatively compact Cartan subgroup of $M^{\dagger}$ and $M^{T}=\left\{x t x^{-1}\right.$ : $\left.x \in M^{\dagger}, t \in T^{\prime}\right\}$. As in [8, 10.10], $\widetilde{\Theta}\left(M^{\dagger}: h: y\right)$ is jointly smooth for 
$(y, h) \in M^{T} \times i v^{*}$. It satisfies the same estimates as the $\Theta\left(M^{\dagger}: h: y\right)$ in Lemma 6.10. Now using the estimates of the proof of Theorem 6.1, we conclude that $\widetilde{\Theta}\left(M^{\dagger}: h: R(m) G_{f}(h: \nu)\right)$ is jointly smooth for $(h, \nu, m) \in i v^{*} \times \mathscr{F} \times M^{\dagger}$. Now clearly $\widetilde{\Psi}_{f}$ is jointly smooth and satisfies (ii). It also satisfies (i) since for $h \in\left(i \mathrm{v}^{*}\right)^{\prime}$ it is a constant multiple of $\Psi_{f}(h: \nu)$. Q.E.D.

Theorem 7.7. Suppose $\Psi \in C^{\infty}\left(i \mathfrak{v}^{*} \times \mathscr{F} \times M^{\dagger}: W\right)$ satisfies

(i) $\Psi(h: \nu) \in \mathscr{S}\left(M^{\dagger}: W: h\right)$ for all $h \in\left(i v^{*}\right)^{\prime}, \nu \in \mathscr{F}$;

(ii) $\Psi(h: \nu) \equiv 0$ for all $h \in\left(i \mathfrak{v}^{*}\right)^{s}, \nu \in \mathscr{F}$.

Then for any $D \in D\left(i \mathfrak{v}^{*} \times \mathscr{F}\right), \Psi(h: \nu ; D) \equiv 0$ for all $h \in\left(i v^{*}\right)^{s}, \nu \in \mathscr{F}$.

We will prove the theorem by reducing it to a result on $M^{\prime}$, the universal covering group of $M^{0}$. We use the notation of $\S 5$. Thus corresponding to each $\mathscr{D} \in \mathscr{C}\left(\lambda_{0}\right)$ we have chambers $\mathscr{D}_{M} \subseteq i v_{M}^{*}$ and $\mathscr{D}_{M^{\prime}} \subseteq i \mathfrak{v}_{M^{\prime}}^{*}$, and the spaces $\mathscr{S}\left(M^{0}: W_{0}: h\right)$ and $\mathscr{S}\left(M^{\prime}: W_{0}: h\right)$ are defined as in (5.8) for $h \in \operatorname{cl}\left(\mathscr{D}_{M}\right)$ or $\operatorname{cl}\left(\mathscr{D}_{M^{\prime}}\right)$ respectively.

Lemma 7.8. Let $E \subseteq i \mathfrak{v}_{M^{\prime}}^{*}$ be any subspace of $i \mathfrak{v}_{M^{\prime}}^{*}$. Suppose

$$
\Psi \in C^{\infty}\left(E \times M^{\prime}: W_{0}\right)
$$

satisfies

(i) $\Psi(h) \in \mathscr{S}\left(M^{\prime}: W_{0}: h\right)$ for all $h \in E \cap\left(i \mathfrak{v}_{M^{\prime}}^{*}\right)^{\prime}$;

(ii) $\Psi(h) \equiv 0$ for all $h \in E \cap\left(i v_{M^{\prime}}\right)^{s}$.

Then given any $D \in D(E), \Psi\left(h_{0} ; D\right) \equiv 0$ for all $h_{0} \in E \cap\left(i \mathfrak{v}_{M^{\prime}}^{*}\right)^{s}$.

Proof. Fix $h_{0} \in E \cap\left(i \mathrm{v}_{M^{\prime}}^{*}\right)^{s}$ and $\alpha \in \Phi_{M}^{+}$so that $\left\langle\lambda_{0}+h_{0}, \alpha\right\rangle=0$. As in $[9,4.6]$ we decompose $\mathfrak{m}=\mathfrak{m}_{0} \oplus \cdots \oplus \mathfrak{m}_{t}$, where $\mathfrak{m}_{0}$ is abelian and the $\mathfrak{m}_{i}$ are simple ideals, and $\mathfrak{v}_{M^{\prime}}=\mathfrak{v}_{0} \oplus \cdots \oplus \mathfrak{v}_{t}$, where $\mathfrak{v}_{i}=\mathfrak{v}_{M^{\prime}} \cap \mathfrak{m}_{i}, 0 \leq i \leq t$. Since $\lambda_{0} \in \Lambda_{M, 0}$, the family $\left\{\lambda_{0}+h: h \in i \mathfrak{v}_{M^{\prime}}^{*}\right\}$ is generically regular. Thus $\alpha$ is not orthogonal to $i \mathfrak{v}_{M^{\prime}}^{*}$, so that $\alpha$ is a root of a simple ideal $\mathfrak{m}_{j}$ for which $\mathfrak{v}_{j} \neq\{0\}$. Thus $\mathfrak{m}_{j}$ must be a noncompact factor of hermitian type, and $\operatorname{dim} \mathfrak{v}_{j}=1$.

Let $p_{j}: i \mathfrak{v}_{M^{\prime}}^{*}=i \mathfrak{v}_{0}^{*} \oplus \cdots \oplus i \mathfrak{v}_{t}^{*} \rightarrow i \mathfrak{v}_{j}^{*}$ denote the projection map. Since $\alpha$ is a root of $\mathfrak{m}_{j},\left\langle\lambda_{0}+h, \alpha\right\rangle=\left\langle\lambda_{0}+p_{j}(h), \alpha\right\rangle$ for all $h \in i \mathfrak{i v}_{M^{\prime}}^{*}$. Suppose $p_{j}(h)=0$ for all $h \in E$. Then $\left\langle\lambda_{0}+h, \alpha\right\rangle=\left\langle\lambda_{0}, \alpha\right\rangle=\left\langle\lambda_{0}+h_{0}, \alpha\right\rangle=0$ for all $h \in E$, so by assumption $\Psi(h) \equiv 0$ for all $h \in E$. Thus $D \Psi(h) \equiv 0$ for all $D \in D(E)$, $h \in E$. Thus we may as well assume that $p_{j}: E \rightarrow i v_{j}^{*}$ is not identically zero, hence is surjective.

Now we can decompose $E=E^{\prime} \oplus E^{\prime \prime}$, where $E^{\prime \prime}$ is $E \cap \operatorname{Ker} p_{j}$ and $E^{\prime}$ is the orthogonal complement of $E^{\prime \prime}$. Further, we can define a linear map $\varphi: i v_{j}^{*} \rightarrow E^{\prime} \subseteq E$ so that $p_{j} \circ \varphi$ is the identity on $i v_{j}^{*}$ and $\varphi \circ p_{j}$ is the identity on $E^{\prime}$. Every $D \in D(E)$ is a finite sum of products $D^{\prime} D^{\prime \prime}$, where $D^{\prime} \in D\left(E^{\prime}\right)$ and $D^{\prime \prime} \in D\left(E^{\prime \prime}\right)$. Thus it is enough to prove the result when $D=D^{\prime} D^{\prime \prime}$. Fix $D^{\prime \prime} \in D\left(E^{\prime \prime}\right), h^{\prime \prime} \in E^{\prime \prime}$, and $x \in M^{\prime \prime}=\prod_{i \neq j} M_{i}$. Then for $h_{j} \in i v_{j}^{*}, x_{j} \in M_{j}$, 
define $\Psi_{j}\left(h_{j}: x_{j}\right)=\left(D^{\prime \prime} \Psi\right)\left(\varphi\left(h_{j}\right)+h^{\prime \prime}: x_{j} x\right)$. Then $\Psi_{j} \in C^{\infty}\left(i v_{j}^{*} \times M_{j}\right)$ and satisfies the hypotheses of the lemma for the simple group $M_{j}$. By [8, 11.11], $D_{j} \Psi_{j}\left(h_{j}: x_{j}\right)=0$ for all $x_{j} \in M_{j}, D_{j} \in D\left(i \mathfrak{v}_{j}^{*}\right)$ if $\left\langle\lambda_{0, j}+h_{j}, \alpha\right\rangle=0$. Now for any $D^{\prime} \in D\left(E^{\prime}\right)$ there is $D_{j} \in D\left(i v_{j}^{*}\right)$ so that for all $h^{\prime} \in E^{\prime}$ and $x_{j} \in M_{j}$, $\left(D^{\prime} D^{\prime \prime} \Psi\right)\left(h^{\prime}+h^{\prime \prime}: x_{j} x\right)=\left(D_{j} \Psi_{j}\right)\left(p_{j}\left(h^{\prime}\right): x_{j}\right)$. Further, if $\left\langle\lambda_{0}+h^{\prime}+h^{\prime \prime}, \alpha\right\rangle=0$, then $\left\langle\lambda_{0, j}+p_{j}\left(h^{\prime}\right), \alpha\right\rangle=0$, so that

$$
\left(D^{\prime} D^{\prime \prime} \Psi\right)\left(h^{\prime}+h^{\prime \prime}: x_{j} x\right)=\left(D_{j} \Psi_{j}\right)\left(p_{j}\left(h^{\prime}\right): x_{j}\right)=0 \text {. Q.E.D. }
$$

Lemma 7.9. Suppose $\Psi \in C^{\infty}\left(i v_{M}^{*} \times M^{0}: W\right)$ satisfies

(i) $\Psi(h) \in \mathscr{S}\left(M^{0}: W_{0}: h\right)$ for all $h \in\left(i v_{M}^{*}\right)^{\prime}$;

(ii) $\Psi(h) \equiv 0$ for all $h \in\left(i v_{M}^{*}\right)^{s}$.

Then given any $D \in D\left(i \mathfrak{v}_{M}^{*}\right), \Psi\left(h_{0} ; D\right) \equiv 0$ for all $h_{0} \in\left(i \mathfrak{v}_{M}^{*}\right)^{s}$.

Proof. Let $E=i v_{M}^{*} \subseteq i v_{M^{\prime}}^{*}$. Define $\Psi^{\prime}: E \times M^{\prime} \rightarrow W_{0}$ by $\Psi^{\prime}\left(h: m^{\prime}\right)=$ $\Psi\left(h: p\left(m^{\prime}\right)\right)$. Then $\Psi^{\prime} \in C^{\infty}\left(E \times M^{\prime}: W_{0}\right)$ satisfies the hypotheses for Lemma 7.8 by Lemma 5.10 . Q.E.D.

Proof of Theorem 7.7. Decompose $i v^{*}=\operatorname{Ker} h_{M} \oplus \varphi\left(i v_{M}^{*}\right)$, where $\varphi: i v_{M}^{*}$ $\rightarrow i v^{*}$ is an injection such that $h_{M} \circ \varphi$ is the identity on $i v_{M}^{*}$. Every $D \in$ $D\left(i \mathfrak{v}^{*} \times \mathscr{F}\right)$ can be written as a finite sum of products $D_{1} D_{2} D_{\nu}$, where $D_{1} \in$ $D\left(\varphi\left(i v_{M}^{*}\right)\right), D_{2} \in D\left(\operatorname{Ker} h_{M}\right)$, and $D_{\nu} \in D(\mathscr{F})$. Thus it is enough to assume $D=D_{1} D_{2} D_{\nu}$. Fix $D_{2} \in D\left(\operatorname{Ker} h_{M}\right), D_{\nu} \in D(\mathscr{F}), h_{2} \in \operatorname{Ker} h_{M}$, and $\nu \in \mathscr{F}$, and for $h_{1} \in i v_{M}^{*}, m^{0} \in M^{0}$, define $\Psi^{0}\left(h_{1}: m^{0}\right)=\left(D_{2} D_{\nu} \Psi\right)\left(\varphi\left(h_{1}\right)+h_{2}\right.$ : $\left.\nu: m^{0}\right)$. Then $\Psi^{0} \in C^{\infty}\left(i \mathfrak{v}_{M}^{*} \times M^{0}: W_{0}\right)$. Further, for all $h_{1} \in i \mathfrak{v}_{M}^{*}$, $\left.\left(h_{2}, \nu\right) \mapsto \Psi\left(\varphi\left(h_{1}\right)+h_{2}: \nu\right)\right|_{M^{0}}$ is a smooth $\mathscr{S}\left(M^{0}: W_{0}: h\right)$-valued function on $\operatorname{Ker} h_{M} \times \mathscr{F}$. Thus $\Psi^{0}\left(h_{1}\right) \in \mathscr{S}\left(M^{0}: W_{0}: h_{1}\right)$ for all $h_{1} \in i v_{M}^{*}$. Further, for $h_{1} \in i_{\mathfrak{v}}^{*}{ }_{M}$ and $\alpha \in \Phi_{M}^{+}$,

$$
\left\langle\lambda_{0}+h_{1}, \alpha\right\rangle=\left\langle\lambda_{0}+h_{M}\left(\varphi\left(h_{1}\right)+h_{2}\right), \alpha\right\rangle \text { for all } h_{2} \in \operatorname{Ker} h_{M} .
$$

Thus, $\varphi\left(h_{1}\right)+h_{2} \in\left(i \mathfrak{v}^{*}\right)^{s}$ if and only if $h_{1} \in\left(i \mathfrak{v}_{M}^{*}\right)^{s}$. Thus, if $h_{1} \in\left(i \mathfrak{v}_{M}^{*}\right)^{s}$, then $\Psi\left(\varphi\left(h_{1}\right)+h_{2}: \nu\right) \equiv 0$ for all $\nu \in \mathscr{F}, h_{2} \in \operatorname{Ker} h_{M}$ by hypothesis (ii). Thus,

$$
\Psi^{0}\left(h_{1}: m^{0}\right)=D_{2} D_{\nu} \Psi\left(\varphi\left(h_{1}\right)+h_{2}: \nu: m^{0}\right)=0 \text { for all } m^{0} \in M^{0} .
$$

Then by Lemma 7.9, $D \Psi^{0}\left(h_{1}\right) \equiv 0$ for all $D \in D\left(i v_{M}^{*}\right)$. But given $D_{1} \in$ $D\left(\varphi\left(i v_{M}^{*}\right)\right)$ there is $D_{1}^{\prime} \in D\left(i v_{M}^{*}\right)$ so that

$$
\left(D_{1} D_{2} D_{\nu} \Psi\right)\left(\varphi\left(h_{1}\right)+h_{2}: \nu: m^{0}\right)=D_{1}^{\prime} \Psi^{0}\left(h_{1}: m^{0}\right)=0
$$

for $\varphi\left(h_{1}\right)+h_{2} \in\left(i \mathfrak{v}^{*}\right)^{s}$. Thus we have proved that given $D \in D\left(i \mathfrak{v}^{*} \times \mathscr{F}\right)$, $(D \Psi)\left(h: \nu: m^{0}\right)=0$ for all $\left(h, \nu, m^{0}\right) \in\left(i v^{*}\right)^{s} \times \mathscr{F} \times M^{0}$. But now for arbitrary $m \in M^{\dagger}, m=z m^{0}$, where $z \in Z_{M}\left(M^{0}\right), m^{0} \in M^{0}$. Then 
$\Psi(h: \nu: m)={ }^{\prime} \tau_{1, h}(z) \Psi\left(h: \nu: m^{0}\right)$ and so there are $D_{i} \in D\left(i v^{*}\right), D_{i}^{\prime} \in$ $D\left(i v^{*} \times \mathscr{F}\right)$, so that

$$
D \Psi(h: \nu: m)=\sum_{i}\left[D_{i}{ }^{\prime} \tau_{1, h}(z)\right] D_{i}^{\prime} \Psi\left(h: \nu: m^{0}\right)
$$

Now $D \Psi(h: \nu: m)=0$ since each $D_{i}^{\prime} \Psi\left(h: \nu: m^{0}\right)=0$ if $h \in\left(i v^{*}\right)^{s}$. Q.E.D.

Corollary 7.10. Suppose $f \in \mathscr{C}_{H}(G)$. Then for any $D \in D\left(i 0^{*} \times \mathscr{F}\right)$, $\widetilde{\Psi}_{f}(h: \nu ; D: m)=0$ for all $(h, \nu, m) \in\left(i v^{*}\right)^{s} \times \mathscr{F} \times M^{\dagger}$.

Let $E$ be a convex open region in $\mathbf{R} \times \mathbf{R}^{n}$ so that in $x \in \mathbf{R}, y \in \mathbf{R}^{n}$ with $(x, y) \in \operatorname{cl}(E)$, then $(t x, y) \in \operatorname{cl}(E)$ for all $0 \leq t \leq 1$. Let $W$ be a finitedimensional vector space. Write $D_{x}=\partial / \partial x$.

Lemma 7.11. Let $f \in C^{\infty}(\operatorname{cl}(E): W)$ such that $D f(0, y)=0$ for all $y \in \mathbf{R}^{n}$ such that $(0, y) \in \operatorname{cl}(E), D \in D\left(\mathbf{R} \times \mathbf{R}^{n}\right)$. Fix $D_{0} \in P\left(\mathbf{R} \times \mathbf{R}^{n}\right)$. Then for every $k \geq 0$, there is $g_{k} \in C^{\infty}(\operatorname{cl}(E): W)$ such that $D_{0} f(x, y)=x^{k} g_{k}(x, y)$, $(x, y) \in E$, and $\left|g_{k}(x, y)\right| \leq \sup _{0 \leq t \leq 1}\left|D_{x}^{k} D_{0} f(t x, y)\right|$.

Proof. Since $D_{0} f$ inherits the properties required of $f$, it is enough to prove the conclusion when $D_{0}=1$. Fix $k>0$. For $(x, y) \in \operatorname{cl}(E)$, define $h_{1}(x, y) \in \int_{0}^{1}\left(D_{x}^{k} f\right)(t x, y) d t$. Clearly $h_{1} \in C^{\infty}(\operatorname{cl}(E): W)$ and $\left|h_{1}(x, y)\right| \leq$ $\sup _{0 \leq t \leq 1}\left|D_{x}^{k} f(t x, y)\right|$. Further, $h_{1}(0, y)=0$ and if $x \neq 0, h_{1}(x, y)=$ $x^{-1} D_{x}^{k-1} f(x, y)$. Thus $x h_{1}=D_{x}^{k-1} f$ for all $x$.

Let $1 \leq j<k$ and assume that we have defined $h_{j} \in C^{\infty}(\operatorname{cl}(E): W)$ so that $h_{j}(0, y)=0, x^{j} h_{j}=D_{x}^{k-j} f$ for all $x$, and $\left|h_{j}(x, y)\right| \leq \sup _{0 \leq t \leq 1}\left|D_{x}^{k} f(t x, y)\right|$. Then we define $h_{j+1}(x, y)=\int_{0}^{1} t^{j} h_{j}(t x, y) d t$. Then $h_{j+1} \in C^{\infty}(\operatorname{cl}(E): W)$ and

$$
\left|h_{j+1}(x, y)\right| \leq \sup _{0 \leq t \leq 1}\left|t^{j} h_{j}(t x, y)\right| \leq \sup _{0 \leq t \leq 1}\left|D_{x}^{k} f(t x, y)\right| .
$$

Also $h_{j+1}(0, y)=0$ and if $x \neq 0$,

$$
h_{j+1}(x, y)=\int_{0}^{1} t^{j} \frac{D_{x}^{k-j} f(x t, y)}{(x t)^{j}} d t=x^{-(j+1)} D_{x}^{k-(j+1)} f(x, y) .
$$

Thus $x^{j+1} h_{j+1}=D_{x}^{k-(j+1)}$ for all $x$. Thus by induction we have $h_{j}, 1 \leq j \leq k$. Now set $g_{k}=h_{k}$. Q.E.D.

Lemma 7.12. Let $s \geq 0$ and $D_{0} \in P\left(i v^{*} \times \mathscr{F}\right)$. Then there is a finite subset $S \subset P\left(\right.$ iv $\left.^{*} \times \mathscr{F}\right)$ so that

$$
\sup _{(h, \nu) \in \mathscr{D} \times \mathscr{F}}\left(1+d(h)^{-1}\right)^{s}\left\|F\left(h: \nu ; D_{0}\right)\right\| \leq 2^{s} \sum_{D^{\prime} \in S} \sup _{(h, \nu) \in \mathscr{D} \times \mathscr{F}}\left\|F\left(h: \nu ; D^{\prime}\right)\right\|
$$

for every $F \in C^{\infty}(\operatorname{cl}(\mathscr{D}) \times \mathscr{F}: W)$ satisfying $(D F)(h: \nu)=0$ for all $h \in\left(i \mathrm{v}^{*}\right)^{s}$, $\nu \in \mathscr{F}, D \in D\left(i \mathrm{v}^{*} \times \mathscr{F}\right)$. 
Proof. For each $\alpha \in \Phi_{M}^{+}$, let $\mathscr{H}_{\alpha}=\left\{h \in i v^{*}:\left\langle\lambda_{0}+h_{M}(h), \alpha\right\rangle=0\right\}$. Then bdry $\mathscr{D}=\operatorname{cl}(\mathscr{D}) \backslash \mathscr{D}=\bigcup_{\alpha}\left(\mathscr{H}_{\alpha} \cap \operatorname{cl}(\mathscr{D})\right)$. Let $\Phi^{\prime}=\left\{\alpha \in \Phi_{M}^{+}: \mathscr{H}_{\alpha} \cap \operatorname{cl}(\mathscr{D})\right.$ is a boundary component of maximal dimension $\}$. For each $\alpha \in \Phi^{\prime}$, let $d_{\alpha}(h)$ denote the distance from $h$ to $\mathscr{H}_{\alpha}$ and let $\mathscr{D}_{\alpha}=\left\{h \in \mathscr{D}: d_{\alpha}(h)=d(h)\right\}$. Then $\mathscr{D}=\bigcup_{\alpha} \mathscr{D}_{\alpha}$.

Now

$$
\begin{aligned}
& \sup _{(h, \nu) \in \mathscr{D} \times \mathscr{F}^{(}}\left(1+d(h)^{-1}\right)^{s}\|F(h: \nu ; D)\| \\
& \quad \leq \sum_{\alpha \in \Phi^{\prime}} \sup _{(h, \nu) \in \mathscr{D}_{\alpha} \times \mathscr{F}^{(}}\left(1+d(h)^{-1}\right)^{s}\|F(h: \nu ; D)\| .
\end{aligned}
$$

Fix $\alpha \in \Phi^{\prime}$ and decompose $\mathscr{D}_{\alpha}=\mathscr{D}_{\alpha}^{\prime} \cup \mathscr{D}_{\alpha}^{\prime \prime}$, where $\mathscr{D}_{\alpha}^{\prime}=\left\{h \in \mathscr{D}_{\alpha}: d_{\alpha}(h)<\right.$ $1\}$ and $\mathscr{D}_{\alpha}^{\prime \prime}=\left\{h \in \mathscr{D}_{\alpha}: d_{\alpha}(h) \geq 1\right\}$. Then for $h \in \mathscr{D}_{\alpha}^{\prime},\left(1+d_{\alpha}(h)^{-1}\right)^{s} \leq$ $2^{s} d_{\alpha}(h)^{-s}$ and for $h \in \mathscr{D}_{\alpha}^{\prime \prime},\left(1+d_{\alpha}(h)^{-1}\right)^{s} \leq 2^{s}$. We can choose coordinates $h=\left(h_{\alpha}, h_{\alpha}^{\prime}, \nu\right) \in i \mathfrak{v}^{*} \times \mathscr{F}$ so that $d_{\alpha}(h)=\left|h_{\alpha}\right|$ and $\mathscr{H}_{\alpha}$ is the hyperplane $h_{\alpha}=0$. Let $E=\left(\mathscr{D}_{\alpha}^{\prime}\right)^{0} \times \mathscr{F}$, where $\left(\mathscr{D}_{\alpha}^{\prime}\right)^{0}$ denotes the interior of $\mathscr{D}_{\alpha}^{\prime}$. Then $E$ satisfies the conditions for Lemma 7.11. Let $D_{\alpha} \in D\left(i \mathfrak{v}^{*} \times \mathscr{F}\right)$ correspond to $\partial / \partial h_{\alpha}$. Then by Lemma 7.11, for all $(h, \nu)=\left(h_{\alpha}, h_{\alpha}^{\prime}, \nu\right) \in E$,

$$
\begin{aligned}
d_{\alpha}(h)^{-s}\|F(h: \nu ; D)\| & \leq \sup _{0 \leq t \leq 1}\left\|F\left(t h_{\alpha}: h_{\alpha}^{\prime}: \nu ; D_{\alpha}^{s} D\right)\right\| \\
& \leq \sup _{(h, \nu) \in E}\left\|F\left(h: \nu ; D_{\alpha}^{s} D\right)\right\| .
\end{aligned}
$$

Thus

$$
\begin{aligned}
\sup _{(h, \nu) \in \mathscr{Q}_{\alpha} \times \mathscr{F}}\left(1+d_{\alpha}(h)^{-1}\right)^{s}\|F(h: \nu ; D)\| \\
\quad \leq 2^{s} \sup _{(h, \nu) \in \mathscr{D}_{\alpha}^{\prime \prime} \times \mathscr{F}}\|F(h: \nu ; D)\|+2^{s} \sup _{(h, \nu) \in \mathscr{D}_{\alpha}^{\prime} \times \mathscr{F}}\left\|F\left(h: \nu ; D_{\alpha}^{s} D\right)\right\| \\
\quad \leq 2^{s}\left[\sup _{(h, \nu) \in \mathscr{D} \times \mathscr{F}}\|F(h: \nu ; D)\|+\sup _{(h, \nu) \in \mathscr{D} \times \mathscr{F}}\left\|F\left(h: \nu ; D_{\alpha}^{s} D\right)\right\|\right] \text {. Q.E.D. }
\end{aligned}
$$

Lemma 7.13. Let $f \in \mathscr{C}_{H}(G)$. Then for any $D \in D\left(i \mathfrak{v}^{*} \times \mathscr{F}\right)$,

$$
D \Psi_{f}\left(h_{0}: \nu: m\right)=0 \text { for all }(\nu, m) \in \mathscr{F} \times M^{\dagger}, h_{0} \in \operatorname{cl}(\mathscr{D}) \cap\left(i v^{*}\right)^{s} .
$$

Proof. Recall (7.5b) for $h \in \mathscr{D}, \Psi_{f}(h: \nu: m)=P(h)^{-1} \widetilde{\Psi}_{f}(h: \nu: m)$, where $P(h)=\varepsilon(\mathscr{D}) \prod_{\alpha \in \Phi_{M}^{+}}\left\langle\alpha, \lambda_{0}+h_{M}(h)\right\rangle$. Let $D \in D\left(i v^{*} \times \mathscr{F}\right)$. Then there are $D_{i} \in P\left(i \mathfrak{v}^{*} \times \mathscr{F}\right)$ and $k_{i} \geq 0$ so that

$$
\Psi_{f}(h: \nu ; D: m)=\sum_{i} P(h)^{-k_{i}} \widetilde{\Psi}_{f}\left(h: \nu ; D_{i}: m\right) .
$$

We use the notation of the proof of Lemma 7.12. Let $h_{0} \in \operatorname{cl}(\mathscr{D}) \cap\left(i \mathrm{v}^{*}\right)^{s}$ and pick $\alpha \in \Phi^{\prime}$ so that $\left\langle\lambda_{0}+h_{M}(h), \alpha\right\rangle=0$. Then we can find $\left\{h_{n}\right\}_{n=1}^{\infty}$ in $D_{\alpha}^{\prime}$ so 
that $\lim _{n \rightarrow \infty} h_{n}=h_{0}$. Since, by Theorem 6.1, $\Psi_{f} \in C^{\infty}\left(\operatorname{cl}(\mathscr{D}) \times \mathscr{F} \times M^{\dagger}\right)$, it follows that

$$
\Psi_{f}\left(h_{0}: \nu ; D: m\right)=\lim _{n \rightarrow \infty} \Psi_{f}\left(h_{n}: \nu ; D: m\right) \text {. }
$$

Now there are constants $C>0, s \geq 0$ so that $|P(h)|^{-1} \leq C d_{\alpha}(h)^{-s}$ for all $h \in \mathscr{D}_{\alpha}$. Thus for all $h \in \mathscr{D}_{\alpha}$,

$$
\begin{aligned}
\left\|P(h)^{-k_{i}} \tilde{\Psi}_{f}\left(h: \nu ; D_{i}: m\right)\right\| & \leq C d_{\alpha}(h)^{-s k_{i}}\left\|\widetilde{\Psi}_{f}\left(h: \nu ; D_{i}: m\right)\right\| \\
& \leq C \sup _{0 \leq t \leq 1}\left\|\widetilde{\Psi}_{f}\left(t h_{\alpha}: h_{\alpha}^{\prime}: \nu ; D_{\alpha}^{s k_{i}} D_{i}: m\right)\right\|,
\end{aligned}
$$

where we use the coordinates $h=\left(h_{\alpha}, h_{\alpha}^{\prime}\right)$. Now if $\left(h_{\alpha}, h_{\alpha}^{\prime}\right) \rightarrow h_{0}=\left(0, h_{\alpha, 0}^{\prime}\right)$,

$$
\sup _{0 \leq t \leq 1}\left\|\widetilde{\Psi}_{f}\left(t h_{\alpha}: h_{\alpha}^{\prime}: \nu ; D_{\alpha}^{s k_{i}} D_{i}: m\right)\right\| \rightarrow\left\|\widetilde{\Psi}_{f}\left(0: h_{\alpha, 0}^{\prime}: \nu ; D_{\alpha}^{s k_{i}} D_{i}: m\right)\right\|=0 .
$$

Q.E.D.

Proof of Theorem 7.2. Let $s \geq 0, D \in P\left(i 0^{*} \times \mathscr{F}\right)$. Then for any $f \in \mathscr{C}_{H}(G)$ and $m \in M^{\dagger},(h, \nu) \rightarrow \Psi_{f}(h: \nu: m)$ satisfies the hypotheses for Lemma 7.12 by Theorem 6.1 and Lemma 7.13. Thus there is a finite subset $S \subseteq P\left(i v^{*} \times \mathscr{F}\right)$ so that

$\sup _{(h, \nu) \in \mathscr{D} \times \mathscr{F}}\left(1+d(h)^{-1}\right)^{s}\left\|\Psi_{f}(h: \nu ; D: m)\right\| \leq \sum_{D^{\prime} \in S} \sup _{(h, \nu) \in \mathscr{D} \times \mathscr{F}}\left\|\Psi_{f}\left(h: \nu ; D^{\prime}: m\right)\right\|$

for all $f \in \mathscr{C}_{H}(G), m \in M^{\dagger}$. Now use Theorem 6.1 for each $D^{\prime} \in S$. Q.E.D.

\section{EXPANSION OF $\Psi_{f}(h: \nu)$ IN TERMS OF HOLOMORPHIC FAMILIES}

In $[9, \S 7]$ we defined a Schwartz space $\mathscr{C}(\mathscr{D} \times \mathscr{F})$ as follows. Let $P\left(i v^{*} \times \mathscr{F}\right)$ denote the set of all polynomial coefficient differential operators on $i v^{*} \times \mathscr{F}$. For $h \in \mathscr{D}$, let $d(h)$ denote the distance from $h$ to the boundary of $\mathscr{D}$. Then (8.1a)

$$
\begin{array}{r}
\mathscr{C}(\mathscr{D} \times \mathscr{F})=\left\{\alpha \in C^{\infty}(\operatorname{cl}(\mathscr{D}) \times \mathscr{F}):\|\alpha\|_{D, t}=\sup _{\mathscr{D} \times \mathscr{F}}\left(1+d(h)^{-1}\right)^{t}|D \alpha(h: \nu)|\right. \\
\left.<\infty \text { for all } t \geq 0, D \in P\left(i v^{*} \times \mathscr{F}\right)\right\} .
\end{array}
$$

We topologize $\mathscr{C}(\mathscr{D} \times \mathscr{F})$ via the seminorms \|\|$_{D, t}$. It is the space of smooth functions on $\operatorname{cl}(\mathscr{D}) \times \mathscr{F}$ which, together with all their derivatives, are rapidly decreasing at infinity and have zeros of infinite order along the walls of $\mathscr{D}$. We also define

$$
\begin{aligned}
\mathscr{C}(\mathscr{D} \times \mathscr{F})_{0}=\left\{\alpha \in C^{\infty}(\operatorname{cl}(\mathscr{D}) \times \mathscr{F}):\|\alpha\|_{D, 0}\right. & =\sup _{\mathscr{D} \times \mathscr{F}}|D \alpha(h: \nu)| \\
& \left.<\infty \text { for all } D \in P\left(i v^{*} \times \mathscr{F}\right)\right\} .
\end{aligned}
$$

Thus functions in $\mathscr{C}(\mathscr{D} \times \mathscr{F})_{0}$ have rapid decay at infinity but are not required to vanish along the walls of $\mathscr{D}$. 
In this section we will use the consistent bases for $\mathscr{S}\left(M^{\prime}: W_{0}: h\right)$ and $\mathscr{S}\left(M^{\prime}: W_{0}: h\right)^{*}$ given in Theorem 5.32 and the estimates for $\Psi_{f}$ given in Theorems 6.1 and 7.2 to prove the following theorem. Define $\mathscr{S}\left(M^{\dagger}: W\right)$ the space of holomorphic families of $K_{M}^{\dagger}$-spherical functions as in (5.2).

Theorem 8.2. There is a finite subset $\left\{F_{p}\right\}$ of $\mathscr{S}\left(M^{\dagger}: W\right)$ satisfying the following. For any $f \in \mathscr{C}(G)$ there are $\left\{\alpha_{p}\right\}$ in $\mathscr{C}(\mathscr{D} \times \mathscr{F})_{0}$ so that $\Psi_{f}(h: \nu)=$ $\sum_{p} \alpha_{p}(h: \nu) F_{p}(h)$ for all $(h, \nu) \in \operatorname{cl}(\mathscr{D}) \times \mathscr{F}$. Moreover, if $f \in \mathscr{C}_{H}(G)$, we can choose the $\alpha_{p} \in \mathscr{C}(\mathscr{D} \times \mathscr{F})$.

Theorem 8.2 has the following important consequence. For each family $F \in$ $\mathscr{S}\left(M^{\dagger}: W\right)$ we can define a family $E(P: F: h: \nu: x)$ of Eisenstein integrals as in (4.5) using $F(h)$ instead of $\Psi_{f}(h: \nu)$. Now combining Theorem 8.2 with formula (4.8) we obtain

Theorem 8.3. Let $f \in \mathscr{C}(G)$. Then there are finitely many $F_{i}$ in $\mathscr{S}\left(M^{\dagger}: W\right)$ and $\alpha_{i} \in \mathscr{C}(\mathscr{D} \times \mathscr{F})_{0}$ so that

$$
\widehat{F}(h: \nu: x)=\sum_{i} \alpha_{i}(h: \nu) E\left(P: F_{i}: h: \nu: x\right)
$$

for all $(h, \nu, x) \in \mathrm{cl}(\mathscr{D}) \times \mathscr{F} \times G$. Moreover, if $f \in \mathscr{C}_{H}(G)$ we can choose the $\alpha_{i}$ in $\mathscr{C}(\mathscr{D} \times \mathscr{F})$.

The remainder of this section is devoted to the proof of Theorem 8.2. We use the notation of $\S 5$. Fix a subset $I \subseteq\{1, \ldots, k\}$ and define $\mathscr{O}_{I}$ as in (5.20). Now fix an open set $J^{\prime} \subseteq \mathscr{O}_{I}$ on which we have consistent bases $\left\{F_{p}\left(h^{\prime}\right)\right\}_{p=1}^{s}$ for $\mathscr{S}\left(M^{\prime}: W_{0}: h^{\prime}\right)$ and $\left\{u_{q}^{*}\left(h^{\prime}\right)\right\}_{q=1}^{s}$ for $\mathscr{S}\left(M^{\prime}: W_{0}: h^{\prime}\right)^{*}, h^{\prime} \in J^{\prime}$, as in Theorem 5.32. Let $J=\left\{h \in \operatorname{cl}(\mathscr{D}): h_{M}(h) \in J^{\prime}\right\}$. Then for any $f \in \mathscr{C}(G)$,

$$
\Psi_{f}^{\prime}(h: \nu)=\left.\Psi_{f}(h: \nu)\right|_{M^{0}} \circ p \in \mathscr{S}\left(M^{\prime}: W_{0}: h_{M}(h)\right),
$$

so there are $\alpha_{p}: J \times \mathscr{F} \rightarrow \mathbf{C}$ so that

$$
\Psi_{f}^{\prime}(h: \nu)=\sum_{p} \alpha_{p}(h: \nu) F_{p}\left(h_{M}(h)\right), \quad(h, \nu) \in J \times \mathscr{F} .
$$

For each $h^{\prime} \in J^{\prime}$, let $M\left(h^{\prime}\right)$ denote the $s \times s$ matrix with entries $m_{p q}\left(h^{\prime}\right)=$ $\left\langle u_{p}^{*}\left(h^{\prime}\right), F_{q}\left(h^{\prime}\right)\right\rangle$. For $h^{\prime} \in J^{\prime}, \operatorname{det} M\left(h^{\prime}\right) \neq 0$ so $M\left(h^{\prime}\right)$ has an inverse $N\left(h^{\prime}\right)$ with entries $n_{p q}\left(h^{\prime}\right)$. Now we can recover the coefficients $\alpha_{p}$ in (8.4a) as

$$
\alpha_{p}(h: \nu)=\sum_{q} n_{p q}\left(h_{M}(h)\right)\left\langle u_{q}^{*}\left(h_{M}(h)\right), \Psi_{f}^{\prime}(h: \nu)\right\rangle .
$$

We first study the properties of the functions $h^{\prime} \rightarrow n_{p q}\left(h^{\prime}\right), h^{\prime} \in J^{\prime}$.

Lemma 8.5. For $1 \leq p, q \leq s, h \rightarrow n_{p q}(h)$ is a smooth function on $J^{\prime}$. For each $D \in D\left(i v_{M^{\prime}}^{*}\right)$, there are constants $C, m \geq 0$ so that $\left|n_{p q}(h ; D)\right| \leq C(1+|h|)^{m}$ for all $h \in J^{\prime}$. 
Proof. Since each $F_{p}(h)$ is of the form $S F(\varphi: h), S \in \operatorname{End}_{K_{M^{\prime}}}\left(W_{0}\right), \varphi \in$ $\mathscr{F}_{I}\left(M^{\prime}: \tau_{1}: \tau_{2}\right)$, and $u_{p}^{*}(h)$ is a finite linear combination of terms

$$
R_{h}\left(\delta(h) \otimes w^{*}\right), \quad \delta \in \mathscr{F}_{I}^{*}, w \in W_{0}^{*},
$$

each entry $m_{p q}(h)$ of $M(h)$ is a finite linear combination of terms of the form $\left\langle\delta(h) \otimes w^{*}, S F(\varphi: h)\right\rangle$. Now as in the proof of Lemma 5.25, $S F(\varphi: h)$ is a finite linear combination of terms of the form $e^{h}(k b)(k \cdot \varphi(h) \cdot b) \otimes w$, where $k, b \in K_{M^{\prime}}, w \in W_{0}$. Thus $m_{p q}(h)$ is a finite linear combination of terms of the form

$$
\begin{aligned}
& e^{h}(k b)\left\langle\delta(h) \otimes w^{*}, k \cdot \varphi(h) \cdot b \otimes w\right\rangle \\
& \quad=e^{h}(k b)\langle\delta(h), k \cdot \varphi(h) \cdot b\rangle\left\langle w^{*}, w\right\rangle .
\end{aligned}
$$

Decompose $M=V_{0} \times M^{\prime}(I) \times \prod_{i \in I} M_{i}, i \mathfrak{v}_{M^{\prime}}^{*}=i \mathfrak{v}_{0}^{*} \oplus i \mathfrak{v}_{I}^{*} \oplus \sum_{i \in I} i \mathfrak{v}_{i}^{*}$ as in Lemma 5.27, where $\mathfrak{v}_{I}=\mathfrak{v}_{M^{\prime}} \cap \mathfrak{m}^{\prime}(I)$. Then we can assume $\varphi \in \mathscr{F}_{I}$ is a product,

$$
\varphi\left(h_{0}+h^{\prime}+\sum_{i \in I} h_{i}: x_{0} x^{\prime} \prod_{i \in I} x_{i}\right)=e^{h_{0}}\left(x_{0}\right) \varphi^{\prime}\left(h^{\prime}: x^{\prime}\right) \prod_{i \in I} \varphi_{i}\left(h_{i}: x_{i}\right),
$$

where $\varphi^{\prime}$ is a holomorphic family of matrix coefficients on $M^{\prime}(I)$ and each $\varphi_{i} \in \mathscr{F}_{I, i}$. Pick $m^{\prime} \in M^{\prime}(I), \psi_{i} \in \mathscr{F}_{I, i}, i \in I$, so that

$$
\delta(h)=\delta\left(m^{\prime}: \prod_{i \in I} \psi_{i}: h\right) .
$$

Then for $k=k_{0} k^{\prime} \prod k_{i}, b=b_{0} b^{\prime} \prod b_{i} \in K_{M^{\prime}}, h=h_{0}+h^{\prime}+\sum h_{i} \in i \mathfrak{v}_{M^{\prime}}^{*}$,

$$
\begin{aligned}
\langle\delta(h), k \cdot \varphi(h) \cdot b\rangle= & e^{h_{0}}\left(k_{0}^{-1} b_{0}^{-1}\right) \varphi^{\prime}\left(h^{\prime}: k^{\prime-1} m^{\prime} b^{\prime-1}\right) \\
& \times \prod_{i \in I} \int_{M_{i} / Z_{i}} \varphi_{i}\left(h_{i}: k_{i}^{-1} x_{i} b_{i}^{-1}\right) \overline{\psi_{i}\left(h_{i}: x_{i}\right)} d\left(x_{i} Z_{i}\right) .
\end{aligned}
$$

Thus,

$$
e^{h}(k b)\langle\delta(h), k \cdot \varphi(h) \cdot b\rangle=f^{\prime}\left(h^{\prime}\right) \prod_{i \in I} f_{i}\left(h_{i}\right),
$$

where $f^{\prime}\left(h^{\prime}\right)=e^{h^{\prime}}\left(k^{\prime} b^{\prime}\right) \varphi^{\prime}\left(h^{\prime}: k^{\prime-1} m^{\prime} b^{-1}\right)$ is a holomorphic function of $h^{\prime} \in$ $\left(i \mathfrak{v}_{I}\right)_{\mathrm{C}}^{*}$, and for $i \in I$,

$$
f_{i}\left(h_{i}\right)=e^{h_{i}}\left(b_{i} k_{i}\right) \int_{M_{i} / Z_{i}} \varphi_{i}\left(h_{i}: k_{i}^{-1} x_{i} b_{i}^{-1}\right) \overline{\psi_{i}\left(h_{i}: x_{i}\right)} d\left(x_{i} Z_{i}\right) .
$$

As in the proof of Lemma 5.23, $\varphi_{i}\left(h_{i}: k_{i}^{-1} x_{i} b_{i}^{-1}\right)$ is a finite linear combination of terms of the form $e^{h_{i}}\left(k_{i}^{-1} b_{i}^{-1}\right) \varphi_{i}^{\prime}\left(h_{i}: x_{i}\right)$, where $\varphi_{i}^{\prime} \in \mathscr{F}_{I, i}$. Thus, $f_{i}\left(h_{i}\right)$ is a finite linear combination of terms $\left\langle\varphi_{i}^{\prime}\left(h_{i}\right), \psi_{i}\left(h_{i}\right)\right\rangle_{L_{2}\left(M_{i} / Z_{i}\right)}$. Now using [8, $6.11,6.16]$ this is a rational function of $h_{i}$ with no poles in $\mathscr{D}_{i}$. Thus we have proved that each $m_{p q}(h)$ is a finite linear combination of terms of the form 
$f\left(h_{0}+h^{\prime}+\sum_{i \in I} h_{i}\right)=f^{\prime}\left(h^{\prime}\right) \prod_{i \in I} R_{i}\left(h_{i}\right)$, where $f^{\prime}$ is holomorphic on $\left(i \mathfrak{v}_{I}\right)_{\mathbf{C}^{\prime}}^{*}$, and each $R_{i}$ is a rational function of $h_{i}$ with no poles in $\mathscr{D}_{i}$. In particular, each $m_{p q}(h)$ is a smooth function of $h \in \operatorname{cl}\left(\mathscr{O}_{I}\right)$ and so each $n_{p q}(h)$ is smooth as long as $\operatorname{det} M(h) \neq 0$, hence for $h \in J^{\prime}$. Further, for any $D \in D\left(i v_{M^{\prime}}^{*}\right)$, there is $k \geq 0$ so that $D n_{p q}(h)$ is a finite linear combination of terms of the form $f^{\prime}\left(h^{\prime}\right) \prod_{i} R_{i}\left(h_{i}\right)(\operatorname{det} M(h))^{-k}$, where $f^{\prime}$ and the $R_{i}$ are as above. Note as in Theorem 5.32 we can assume that $J^{\prime}$ was chosen so that $|\operatorname{det} M(h)| \geq \varepsilon>0$ on $J^{\prime}$. Further, $J^{\prime}=i v_{0}^{*} \times J(I) \times \prod_{i \in I} \mathscr{D}_{i}^{n}$, where $\operatorname{cl}(J(I)) \subseteq i v_{I}^{*}$ is compact. Thus $\left|f^{\prime}\left(h^{\prime}\right)\right|$ is bounded on $\operatorname{cl}\left(J^{\prime}\right)$. Finally, each $R_{i}\left(h_{i}\right)$ is of polynomial growth on $\mathscr{D}_{i}^{n}$ and its poles are outside $\operatorname{cl}\left(\mathscr{D}_{i}^{n}\right) \subseteq \mathscr{D}_{i}$. Thus $D n_{p q}(h)$ satisfies the required estimate. Q.E.D.

As before, assume $J^{\prime} \subseteq \mathscr{O}_{I}$ and $J=\left\{h \in \operatorname{cl}(\mathscr{D}): h_{M}(h) \in J^{\prime}\right\}$.

Lemma 8.6. Let $\varphi \in C^{\infty}\left(J \times \mathscr{F} \times M^{\prime}\right)$ so that $\varphi(h: \nu) \in \mathscr{V}\left(M^{\prime}: \tau_{1}: \tau_{2}: h_{M}(h)\right)$ for all $(h, \nu) \in J \times \mathscr{F}$ and $\varphi$ satisfies one of the following growth estimates: For every $r \geq 0$ and $D \in P\left(i v^{*} \times \mathscr{F}\right)$, there are $C, t \geq 0$ so that

(i) $\sup _{(h, \nu) \in J \times \mathscr{F}}\left|\varphi\left(h: \nu ; D: m^{\prime}\right)\right| \leq C\left(1+\tilde{\sigma}_{M}\left(p\left(m^{\prime}\right)\right)\right)^{t} \Xi_{M}\left(p\left(m^{\prime}\right)\right)^{-1}$ for all $m^{\prime} \in M^{\prime}$

(ii) $\sup _{(h, \nu) \in J \times \mathscr{F}}\left(1+d(h)^{-1}\right)^{r}\left|\varphi\left(h: \nu ; D: m^{\prime}\right)\right|$ $\leq C\left(1+\tilde{\sigma}_{M}\left(p\left(m^{\prime}\right)\right)\right)^{t} \Xi_{M}\left(p\left(m^{\prime}\right)\right)^{-1}$ for all $m^{\prime} \in M^{\prime}$.

Let $\delta \in \mathscr{F}^{*}$ and set $\alpha(h: \nu)=\left\langle\delta\left(h_{M}(h)\right), \varphi(h: \nu)\right\rangle$. Let $D \in P\left(i v^{*} \times \mathscr{F}\right)$ and $r \geq 0$. Then if $\varphi$ satisfies (i), $\sup _{(h, \nu) \in J \times \mathscr{F}}|\alpha(h: \nu ; D)|<\infty$ and if $\varphi$ satisfies (ii), $\sup _{(h, \nu) \in J \times \mathscr{F}}\left(1+d(h)^{-1}\right)^{r}|\alpha(h: \nu ; D)|<\infty$.

Proof. Suppose $\delta(h)=\delta\left(m^{\prime}: \prod \psi_{i}\right), m^{\prime} \in M^{\prime}(I), \psi_{i} \in \mathscr{F}_{I, i}, i \in I$. Then

$$
\alpha(h: \nu)=\int_{\prod M_{i} / Z_{i}} \varphi\left(h: \nu: 1: m^{\prime}: \prod x_{i}\right) \prod \overline{\psi_{i}\left(h_{i}: x_{i}\right)} \prod d\left(x_{i} Z_{i}\right),
$$

where $h_{i}$ is the component of $h_{M}(h)$ in $i v_{i}^{*}$. Write the Cartan decomposition $M_{i}=K_{i} \operatorname{cl}\left(A_{i}^{+}\right) K_{i}$ and $K_{i}=Z_{K_{i}}^{0}\left[K_{i}, K_{i}\right]$ in the notation of $[8,11.13]$. Then as in $[8,11.13]$,

$$
\begin{aligned}
\alpha(h: \nu)=\int \varphi\left(h: \nu: 1: m^{\prime}: \prod_{i}\left(k_{i} a_{i} k_{i}^{\prime}\right)\right) \prod_{i} \overline{\psi_{i}\left(h_{i}: k_{i} a_{i} k_{i}^{\prime}\right)} & D\left(a_{i}\right) \\
& \times \prod_{i} d a_{i} d k_{i} d k_{i}^{\prime},
\end{aligned}
$$

where we integrate over $a_{i} \in \operatorname{cl}\left(A_{i}^{+}\right), k_{i}, k_{i}^{\prime} \in\left[K_{i}, K_{i}\right], i \in I$. Thus, 
$|\alpha(h: \nu ; D)|$ is bounded by a finite sum of terms of the form

$$
\begin{aligned}
\int\left|\varphi\left(h: \nu ; D^{\prime}: 1: m^{\prime}: \prod_{i}\left(k_{i} a_{i} k_{i}^{\prime}\right)\right) \prod_{i} \psi_{i}\left(h_{i} ; D_{i}: k_{i} a_{i} k_{i}^{\prime}\right)\right| & D\left(a_{i}\right) \\
& \times \prod_{i} d a_{i} d k_{i} d k_{i}^{\prime},
\end{aligned}
$$

where $D^{\prime} \in P\left(i \mathrm{v}^{*} \times \mathscr{F}\right)$ and $D_{i} \in D\left(i \mathrm{v}_{i}^{*}\right), i \in I$.

Assume $i v_{i}^{*}=\mathbf{R} h_{i, 0}$ is parametrized so that $\mathscr{D}_{i}=\left\{t h_{i 0}: t>0\right\}$. Then we can assume the decomposition $\mathscr{D}_{i}=\mathscr{D}_{i}^{c} \cup \mathscr{D}_{i}^{n}$ in (5.20) was chosen so that if $b_{i}$ is chosen as in $[8,11.13] \mathscr{D}_{i}^{n} \subseteq\left\{t h_{i, 0}: t \geq b_{i}\right\}$. Now for $h \in J$, $h_{M}(h) \in J^{\prime} \subseteq \mathscr{O}_{I}$, so $h_{i} \in \mathscr{D}_{i}^{n}$. Now as in [7, 5.2], for all $h_{i} \in \mathscr{D}_{i}^{n}$,

$$
\left|\psi_{i}\left(h_{i}: k_{i} a_{i} k_{i}^{\prime}\right)\right| \leq C_{i}\left(1+\left|h_{i}\right|\right)^{d_{i}}\left(1+\sigma\left(a_{i}\right)\right)^{q_{i}} e^{-\left(\lambda_{0, i}+b_{i} h_{i, 0}\right)}\left(\kappa\left(a_{i}\right)\right) .
$$

But assuming that $\varphi$ satisfies (ii), there is $t$ so that

$$
\begin{aligned}
& \sup _{(h, \nu) \in J \times \mathscr{F}}\left(1+d(h)^{-1}\right)^{r}\left|\varphi\left(h: \nu ; D^{\prime}: 1: m^{\prime}: \prod k_{i} a_{i} k_{i}^{\prime}\right)\right| \prod_{i}\left(1+\left|h_{i}\right|\right)^{d_{i}} \\
& \quad \leq C\left(1+\tilde{\sigma}_{M}\left(p\left(m^{\prime} \prod k_{i} a_{i} k_{i}^{\prime}\right)\right)\right)^{t} \Xi_{M}^{-1}\left(p\left(m^{\prime}\right) \prod a_{i}\right) .
\end{aligned}
$$

But since $m^{\prime}$ is fixed and $k_{i}, k_{i}^{\prime} \in K_{M, 1}$, this is bounded by $C^{\prime} \prod_{i}\left(1+\sigma\left(a_{i}\right)\right)^{t} \Xi_{M_{i}}^{-1}\left(a_{i}\right)$. Thus

$$
\begin{aligned}
& \sup _{(h, \nu) \in J \times \mathscr{F}^{-}}\left(1+d(h)^{-1}\right)^{r}|\alpha(h: \nu ; D)| \\
& \quad \leq C^{\prime} \prod_{i} \int_{\mathrm{cl}\left(A_{i}^{+}\right)}\left(1+\sigma\left(a_{i}\right)\right)^{q_{i}+t} \Xi_{M_{i}}^{-1}\left(a_{i}\right) D\left(a_{i}\right) e^{-\left(\lambda_{0, i}+b_{i} h_{i, 0}\right)}\left(\kappa\left(a_{i}\right)\right) d a_{i} \\
& \quad<\infty
\end{aligned}
$$

by $[8,11.3]$. If $\varphi$ satisfies (i), we do the same estimate, omitting the terms $\left(1+d(h)^{-1}\right)^{r}$. Q.E.D.

Lemma 8.7. Suppose $\alpha_{p}: J \times \mathscr{F} \rightarrow \mathbf{C}, 1 \leq p \leq s$, are defined as in (8.4a). Then for any $r \geq 0$ and $D \in P\left(i v^{*} \times \mathscr{F}\right)$,

(i) $\sup _{(h, \nu) \in J \times \mathscr{F}}\left|\alpha_{p}(h: \nu ; D)\right|<\infty$ if $f \in \mathscr{C}(G)$;

(ii) $\sup _{(h, \nu) \in J \times \mathscr{F}}\left(1+d(h)^{-1}\right)^{r}\left|\alpha_{p}(h: \nu ; D)\right|<\infty$ if $f \in \mathscr{C}_{H}(G)$.

Proof. Using (8.4b), there are $D_{i}^{\prime} \in D\left(i v_{M^{\prime}}^{*}\right), D_{i}^{\prime \prime} \in P\left(i v^{*} \times \mathscr{F}\right)$ so that

$$
\alpha_{p}(h: \nu ; D)=\sum_{i, q} n_{p q}\left(h_{M}(h) ; D_{i}^{\prime}\right) \beta_{q}\left(h: \nu ; D_{i}^{\prime \prime}\right),
$$

where $\beta_{q}(h: \nu)=\left\langle u_{q}^{*}\left(h_{M}(h)\right), \Psi_{f}^{\prime}(h: \nu)\right\rangle$. Now by (8.5) there are $C, m \geq 0$ so that

$$
\left|n_{p q}\left(h_{M}(h) ; D_{i}^{\prime}\right)\right| \leq C\left(1+\left|\left(h_{M}(h)\right)\right|\right)^{m} \leq C(1+|h|)^{m}
$$


for all $p, q, i, h \in J$. The polynomial term can be combined with the $D_{i}^{\prime \prime}$, so it suffices to show that

$$
\sup _{(h, \nu) \in J \times \mathscr{F}}\left|\beta_{q}(h: \nu ; D)\right|<\infty \text { for all } D \in P\left(i 0^{*} \times \mathscr{F}\right), 1 \leq q \leq s,
$$

if $f \in \mathscr{C}(G)$, or that

$$
\sup _{(h, \nu) \in J \times \mathscr{F}}\left(1+d(h)^{-1}\right)^{r}\left|\beta_{q}(h: \nu ; D)\right|<\infty
$$

if $f \in \mathscr{C}_{H}(G)$.

But $u_{q}^{*}\left(h_{M}(h)\right)$ is a finite linear combination of terms $\delta\left(h_{M}(h)\right) \otimes w^{*}, \delta \in$ $\mathscr{F}_{I}^{*}, w^{*} \in W_{0}^{*}$. Fix $\delta \in \mathscr{F}_{I}^{*}, w^{*} \in W_{0}^{*}$ and let

$$
\beta(h: \nu)=\left\langle\delta\left(h_{M}(h)\right) \otimes w^{*}, \Psi_{f}^{\prime}(h: \nu)\right\rangle .
$$

Then

$$
\beta(h: \nu)=\left\langle\delta\left(h_{M}(h)\right), \Psi_{f}^{\prime}\left(w^{*}: h: \nu\right)\right\rangle,
$$

where

$$
\Psi_{f}^{\prime}\left(w^{*}: h: \nu\right) \in \mathscr{V}\left(M^{\prime}: \tau_{1}: \tau_{2}: h_{M}(h)\right)
$$

is defined by $\Psi_{f}^{\prime}\left(w^{*}: h: \nu: m^{\prime}\right)=\left\langle w^{*}, \Psi_{f}^{\prime}\left(h: \nu: m^{\prime}\right)\right\rangle,\left(h, \nu, m^{\prime}\right) \epsilon$ $J \times \mathscr{F} \times M^{\prime}$. Now $\Psi_{f}^{\prime}\left(w^{*}\right) \in C^{\infty}\left(J \times \mathscr{F} \times M^{\prime}\right)$ and for all $D \in P\left(i \mathrm{v}^{*} \times \mathscr{F}\right)$,

$$
\begin{aligned}
\left|\Psi_{f}^{\prime}\left(w^{*}: h: \nu ; D: m^{\prime}\right)\right| & \leq\left\|w^{*}\right\|_{\mathrm{op}}\left\|\Psi_{f}^{\prime}\left(h: \nu ; D: m^{\prime}\right)\right\| \\
& =\left\|w^{*}\right\|_{\mathrm{op}}\left\|\Psi_{f}\left(h: \nu ; D: p\left(m^{\prime}\right)\right)\right\| \\
& \leq\left\|w^{*}\right\|_{\mathrm{op}}\left(1+\tilde{\sigma}\left(p\left(m^{\prime}\right)\right)^{q} \Xi_{M}^{-1}\left(p\left(m^{\prime}\right)\right) \mu(f)\right.
\end{aligned}
$$

for all $(h, \nu) \in J \times \mathscr{F}, m^{\prime} \in M^{\prime}$, by Theorem 6.1 if $f \in \mathscr{C}(G)$. Thus $\Psi_{f}^{\prime}\left(w^{*}\right)$ satisfies estimate (i) of Lemma 8.6. Similarly, if $f \in \mathscr{C}_{H}(G)$, we see using Theorem 7.2 that $\Psi_{f}^{\prime}\left(w^{*}\right)$ satisfies estimate (ii) of Lemma 8.6. Thus using Lemma 8.6, $\beta$ satisfies (i) if $f \in \mathscr{C}(G)$ and (ii) if $f \in \mathscr{C}_{H}(G)$. Q.E.D.

For each $I \subseteq\{1, \ldots, k\}$, let $\mathscr{J}_{I}$ be the open cover of $\mathscr{O}_{I}$ given by Theorem 5.32. Let $\mathscr{J}=\bigcup_{I} \mathscr{J}_{I}$. It is a finite open cover of $\operatorname{cl}\left(\mathscr{D}_{M^{\prime}}\right)$.

Lemma 8.8. There is a smooth partition of unity $\{\beta(J)\}_{J \in \mathcal{J}}$ of $\mathrm{cl}\left(\mathscr{D}_{M^{\prime}}\right)$ subordinate to $\mathcal{J}$ so that for each $J \in \mathcal{J}$ and $D \in D\left(i v_{M^{\prime}}{ }^{\prime}\right.$,

$$
\sup _{h \in \mathrm{cl}\left(\mathscr{D}_{M^{\prime}}\right)}|\beta(J: h ; D)|<\infty .
$$

Proof. For each $1 \leq i \leq k$, let $\beta_{i}^{c}, \beta_{i}^{n} \in C^{\infty}\left(\operatorname{cl}\left(\mathscr{D}_{i}\right)\right)$ satisfy

(i) $\operatorname{supp} \beta_{i}^{c} \subseteq \mathscr{D}_{i}^{c}$ and $\operatorname{supp} \beta_{i}^{n} \subseteq \mathscr{D}_{i}^{n}$;

(ii) $\beta_{i}^{c}\left(h_{i}\right)+\beta_{i}^{n}\left(h_{i}\right)=1$ for all $h_{i} \in \operatorname{cl}\left(\mathscr{D}_{i}\right)$;

(iii) $0 \leq \beta_{i}^{c}\left(h_{i}\right), \beta_{i}^{n}\left(h_{i}\right) \leq 1$ for all $h_{i} \in \operatorname{cl}\left(\mathscr{D}_{i}\right)$. 
Note if $\mathscr{D}_{i} \in D\left(i \mathrm{v}_{i}^{*}\right),\left|\beta_{i}^{c}\left(h_{i} ; D_{i}\right)\right|$ is bounded on $\operatorname{cl}\left(\mathscr{D}_{i}\right)$ since it is supported in the compact set $\operatorname{cl}\left(\mathscr{D}_{i}^{c}\right)$, and $\left|\beta_{i}^{n}\left(h_{i} ; D_{i}\right)\right|$ is bounded on $\operatorname{cl}\left(\mathscr{D}_{i}\right)$ since it is bounded on $\operatorname{cl}\left(\mathscr{D}_{i}^{c}\right)$ and is constant on $\operatorname{cl}\left(\mathscr{D}_{i}\right) \backslash \mathscr{D}_{i}^{c}$.

Now we obtain a smooth partition of unity for $\operatorname{cl}\left(\mathscr{D}_{M^{\prime}}\right)$ subordinate to $\left\{\mathscr{O}_{I}\right\}$ by defining $\beta_{I}(h)=\prod_{i \notin I} \beta_{i}^{c}\left(h_{i}\right) \prod_{i \in I} \beta_{i}^{n}\left(h_{i}\right)$, where $h_{i}$ denotes the component of $h$ in $i v_{i}^{*}, 1 \leq i \leq k$. Further, for any $D \in D\left(i \mathfrak{v}_{M^{\prime}}^{*}\right),\left|\beta_{I}(h ; D)\right|$ is bounded on $\operatorname{cl}\left(\mathscr{D}_{M^{\prime}}\right)$.

Fix $I \subseteq\{1, \ldots, k\}$. Each $J \in \mathscr{J}_{I}$ is of the form $J=i \mathfrak{v}_{0}^{*} \times J(I) \times \prod_{i \in I} \mathscr{D}_{i}^{n}$, where $J(l)$ is an open subset of $\operatorname{cl}\left(\mathscr{D}^{\prime}\right) \times \prod_{i \notin I} \mathscr{D}_{i}^{c}$. Now $\left\{J(I): J \in \mathscr{J}_{I}\right\}$ is an open cover of $\operatorname{cl}\left(\mathscr{D}^{\prime}\right) \times \prod_{i \notin I} \operatorname{cl}\left(\mathscr{D}_{i}^{c}\right)$. Let $\{\tilde{\beta}(J(I))\}$ be a smooth partition of unity of $\operatorname{cl}\left(\mathscr{D}^{\prime}\right) \times \prod_{i \notin I} \mathscr{D}_{i}^{c}$ subordinate to $\left\{J(I): J \in \mathscr{J}_{I}\right\}$ such that each $\tilde{\beta}(J(I))$ extends smoothly to $\operatorname{cl}\left(\mathscr{D}^{\prime}\right) \times \prod_{i \notin I} \operatorname{cl}\left(\mathscr{D}_{i}^{c}\right)$. We obtain a partition of unity of $\mathscr{O}_{I}=i v_{0}^{*} \times \operatorname{cl}\left(\mathscr{D}^{\prime}\right) \times \prod_{i \notin I} \mathscr{D}_{i}^{c} \times \prod_{i \in I} \mathscr{D}_{i}^{n}$ subordinate to $\mathscr{J}_{I}$ as follows. If $J=i v_{0}^{*} \times J(I) \times \prod_{i \in I} \mathscr{D}_{i}^{n}$, define $\tilde{\beta}(J: h)=\tilde{\beta}\left(J(I): h^{\prime}\right)$ if $h^{\prime}$ is the component of $h$ in $\operatorname{cl}\left(\mathscr{D}^{\prime}\right) \times \prod_{i \notin I} \mathscr{D}_{i}^{c}$. For each $D \in D\left(i v_{M^{\prime}}^{*}\right),|\tilde{\beta}(J: h ; D)|$ is bounded on $\mathscr{O}_{I}$ since $\beta(J)$ extends smoothly to $\operatorname{cl}\left(\mathscr{O}_{I}\right)$ and depends only on the component of $h$ in the compact set $\operatorname{cl}\left(\mathscr{D}^{\prime}\right) \times \prod_{i \notin I} \mathscr{D}_{i}^{c}$.

Combining the above we obtain a smooth partition of unity of $\operatorname{cl}\left(\mathscr{D}_{M^{\prime}}\right)$ subordinate to $\mathscr{J}=\bigcup \mathscr{J}_{I}$ by setting $\beta(J: h)=\beta_{I}(h) \tilde{\beta}(J: h)$ if $J \in \mathscr{F}_{I}$. Since the derivatives of both $\beta_{I}$ and $\tilde{\beta}(J)$ are bounded, so are those of $\beta(J)$. Q.E.D.

Proof of Theorem 8.2. Let $\left\{\beta\left(J^{\prime}\right)\right\}_{J^{\prime} \in \mathcal{J}}$ be the partition of unity of $\operatorname{cl}\left(\mathscr{D}_{M^{\prime}}\right)$ given by Lemma 8.8. Thus for any $f \in \mathscr{C}(G)$ and $(h, \nu) \in \operatorname{cl}(\mathscr{D}) \times \mathscr{F}$, we can write

$$
\Psi_{f}^{\prime}(h: \nu)=\sum_{J^{\prime} \in \mathcal{J}} \beta\left(J^{\prime}: h_{M}(h)\right) \Psi_{f}^{\prime}(h: \nu) .
$$

For each $J^{\prime} \in \mathscr{J}$, let $\left\{F_{p}^{\prime}\left(J^{\prime}: h^{\prime}\right)\right\}_{p=1}^{s}$ be a basis for $\mathscr{S}\left(M^{\prime}: W_{0}: h^{\prime}\right)$, $h^{\prime} \in J^{\prime}$, as in Theorem 5.32. Then for $J=\left\{h \in \operatorname{cl}(\mathscr{D}): h_{M}(h) \in J^{\prime}\right\}$, we have as in $(8.4 \mathrm{a}) \alpha_{p}\left(J^{\prime}\right): J \times \mathscr{F} \rightarrow \mathbf{C}$ so that

$$
\Psi_{f}^{\prime}(h: \nu)=\sum_{p=1}^{s} \alpha_{p}\left(J^{\prime}: h: \nu\right) F_{p}^{\prime}\left(J^{\prime}: h_{M}(h)\right), \quad(h, \nu) \in J \times \mathscr{F} .
$$

Combining the above we write for all $(h, \nu) \in \operatorname{cl}(\mathscr{D}) \times \mathscr{F}$,

$$
\Psi_{f}^{\prime}(h: \nu)=\sum_{J^{\prime}} \sum_{p} \alpha_{p}^{\prime}\left(J^{\prime}: h: \nu\right) F_{p}^{\prime}\left(J^{\prime}: h_{M}(h)\right),
$$

where

$$
\alpha_{p}^{\prime}\left(J^{\prime}: h: \nu\right)= \begin{cases}\beta\left(J^{\prime}: h_{M}(h)\right) \alpha_{p}\left(J^{\prime}: h: \nu\right) & \text { if } h_{M}(h) \in J^{\prime}, \\ 0 & \text { if } h_{M}(h) \notin J^{\prime}\end{cases}
$$

Now for any $D \in P\left(i 0^{*} \times \mathscr{F}\right), \alpha_{p}^{\prime}\left(J^{\prime}: h: \nu ; D\right)=0$ if $h \notin J$ and there are 


$$
\begin{aligned}
& D_{i}^{\prime} \in D\left(i \mathfrak{v}_{M^{\prime}}^{*}\right), D_{i}^{\prime \prime} \in P\left(i \mathfrak{v}^{*} \times \mathscr{F}\right) \text { so that } \\
& \qquad\left|\alpha_{p}^{\prime}\left(J^{\prime}: h: \nu ; D\right)\right| \leq \sum_{i}\left|\beta\left(J^{\prime}: h_{M}(h) ; D_{i}^{\prime}\right)\right|\left|\alpha_{p}\left(J^{\prime}: h: \nu ; D_{i}^{\prime \prime}\right)\right|
\end{aligned}
$$

if $h \in J$. But each $\left|\beta\left(J^{\prime}: h_{M}(h) ; D_{i}^{\prime}\right)\right|$ is bounded. Then using Lemma 8.7, $\alpha_{p}^{\prime}\left(J^{\prime}\right)$ is in $\mathscr{C}(\mathscr{D} \times \mathscr{F})_{0}$ if $f \in \mathscr{C}(G)$ and is in $\mathscr{C}(\mathscr{D} \times \mathscr{F})$ if $f \in \mathscr{C}_{H}(G)$.

Fix $J^{\prime} \in \mathscr{J}_{I} \subseteq \mathscr{J}$ and $1 \leq p \leq s$. Then there are $\varphi^{\prime} \in \mathscr{F}_{I}\left(M^{\prime}: \tau_{1}: \tau_{2}\right)$ and $S \in \operatorname{End}_{K_{M^{\prime}}}\left(W_{0}\right)$ so that $F_{p}^{\prime}\left(J^{\prime}\right)=S F\left(\varphi^{\prime}\right)$. By (5.12) and (5.21), there are finitely many $\varphi_{q}^{\prime} \in \mathscr{F}\left(M^{\prime}: \tau_{1}: \tau_{2}\right)$ and rational functions $r_{q}$ with no poles in $\operatorname{cl}\left(\mathscr{O}_{I}\right)$ so that $F_{p}^{\prime}\left(J^{\prime}: h^{\prime}\right)=\sum_{q} r_{q}\left(h^{\prime}\right) S F\left(\varphi_{q}^{\prime}: h^{\prime}\right), h^{\prime} \in \mathscr{O}_{I}$. Further, by Proposition 5.33 there are $F_{q}=F_{p q}\left(J^{\prime}\right) \in \mathscr{S}\left(M^{\dagger}: W\right)$ so that $\left.F_{q}(h)\right|_{M^{0}} \circ p=$ $S F\left(\varphi_{q}^{\prime}: h_{M}(h)\right)$ for all $h \in \operatorname{cl}(\mathscr{D})$. Thus,

$$
\alpha_{p}^{\prime}\left(J^{\prime}: h: \nu\right) F_{p}^{\prime}\left(J^{\prime}: h_{M}(h)\right)=\sum_{q} \alpha_{p q}\left(J^{\prime}: h: \nu\right)\left(\left.F_{p q}\left(J^{\prime}: h\right)\right|_{M^{0}} \circ p\right),
$$

where $\alpha_{p q}\left(J^{\prime}: h: \nu\right)=r_{q}\left(h_{M}(h)\right) \alpha_{p}^{\prime}\left(J^{\prime}: h: \nu\right)$. Since $r_{q}$ is a rational function with no poles in the support of $\alpha_{p}^{\prime}\left(J^{\prime}\right), \alpha_{p q}\left(J^{\prime}: h: \nu\right)$ is in $\mathscr{C}(\mathscr{D} \times \mathscr{F})_{0}$ if $f \in \mathscr{C}(G)$ and is in $\mathscr{C}(\mathscr{D} \times \mathscr{F})$ if $f \in \mathscr{C}_{H}(G)$. Finally, since $\left.F \rightarrow F\right|_{M^{0}} \circ p$ is a bijection between $\mathscr{S}\left(M^{\dagger}: W: h\right)$ and $\mathscr{S}\left(M^{\prime}: W_{0}: h_{M}(h)\right)$ for $h \in \operatorname{cl}(\mathscr{D})$, we have

$$
\Psi_{f}(h: \nu)=\sum_{J^{\prime}} \sum_{p} \sum_{q} \alpha_{p q}\left(J^{\prime}: h: \nu\right) F_{p q}\left(J^{\prime}: h\right)
$$

for all $(h, \nu) \in \operatorname{cl}(\mathscr{D}) \times \mathscr{F}$. Q.E.D.

\section{A CHARACTER IDENTITY}

In this section we will prove a generalization of Schmid's character identity identifying the sum of two limits of discrete series with a reducible representation induced from a maximal parabolic subgroup. As a consequence of this basic identity, we will derive character formulas which should give all the matching conditions among the series of tempered representations which are necessary to describe the dual of the Schwartz space.

Assume that $G$ is a connected reductive group with rank $G=\operatorname{rank} K$. Let $B$ be a maximal relatively compact Cartan subgroup of $G$ and let $\Phi=\Phi\left(\mathfrak{g}_{\mathrm{C}}, \mathfrak{b}_{\mathrm{C}}\right)$, $\rho$ the half-sum of positive roots in $\Phi, \Lambda=\left\{\lambda \in i \mathfrak{b}^{*}: \lambda-\rho\right.$ is integral $\}$. Let $\mathscr{C}$ be a chamber of $i b^{*}$ with respect to $\Phi$. Then every $\lambda \in \Lambda \cap \mathrm{cl}(\mathscr{C})$ corresponds to a relative discrete series or limit of relative discrete series representation $\pi_{\lambda}$ of $G$ with character $\Theta(\lambda)$ given roughly as follows (see [5, $\S 2]$ for details).

Let $H$ be a $\theta$-stable Cartan subgroup of $G$ and let $c$ be a Cayley transform with $c\left(\mathfrak{b}_{\mathbf{C}}\right)=\mathfrak{h}_{\mathbf{C}}$. There is a subgroup $W_{H}$ of the Weyl group of $\Phi$ so that $e^{c(w \lambda-\rho)}$ gives a well-defined character of $H$ for $w \in W_{H}, \lambda \in \Lambda$. Now for every $w \in W_{H}$ and chamber $\mathscr{C}$ there is a locally constant function $c(w: \mathscr{C})$ 
on $H^{\prime}$, the set of regular elements in $H$, so that for all $\lambda \in \Lambda \cap \operatorname{cl}(\mathscr{C}), x \in H^{\prime}$,

$$
\Theta(\lambda: x)=\Delta(x)^{-1} \sum_{w \in W_{H}} c(w: \mathscr{C}: x) e^{c(w \lambda)}(x) .
$$

Here $\Delta$ is the Weyl denominator.

Now for any $\lambda \in \Lambda$ and any chamber $\mathscr{C}, \lambda$ not necessarily in $\mathscr{C}$, we can define the coherently continued relative discrete series character

$$
\Theta(\lambda: \mathscr{C}: x)=\Delta(x)^{-1} \sum_{w \in W_{H}} c(w: \mathscr{C}: x) e^{c(w \lambda)}(x), \quad x \in H^{\prime} .
$$

Now let $\beta$ be a singular imaginary root in $\Phi$ which is simple for the ordering of $\Phi$ determining $\mathscr{C}$. Thus $\mathscr{C}$ and $s_{\beta} \mathscr{C}$ are separated only by the wall $\mathscr{H}_{\beta}=$ $\left\{\lambda \in i \mathfrak{b}^{*}:\langle\lambda, \beta\rangle=0\right\}$. (Here $s_{\beta}$ denotes the reflection in $\mathscr{H}_{\beta}$.) Let $H=T \times A$ be the Cartan subgroup of $G$ obtained by the Cayley transform $c$ with respect to $\beta$ and let $P=M A N$ be a corresponding (maximal) cuspidal parabolic subgroup. Then $\mathfrak{t}=\{H \in \mathfrak{b}: \beta(H)=0\}$ and $\mathfrak{a}=\exp \left(\mathbf{R}^{c} H_{\beta}^{*}\right)$. Since $\operatorname{dim} \mathfrak{a}=$ $1, T \subseteq B$ even though it need not be connected. Thus given $\lambda \in \Lambda$ we obtain data for a coherently continued induced character as follows. Let

$$
\lambda_{\mathrm{t}}=\left.\lambda\right|_{\mathrm{t}}
$$

and let $\mathscr{C}_{\mathrm{t}}$ be the chamber of $i \mathrm{t}^{*}$ with respect to $\Phi_{M}=\Phi\left(\mathfrak{m}_{\mathrm{C}}, \mathfrak{t}_{\mathrm{C}}\right)$ such that $\left.\tau\right|_{\mathfrak{t}} \in \mathscr{C}_{\mathfrak{t}}$ for all $\tau \in \mathscr{C} \cup s_{\beta}(\mathscr{C})$. Define $\Theta\left(M^{0}: \lambda_{\mathfrak{t}}: \mathscr{C}_{\mathfrak{t}}\right)$ to be the coherently continued relative discrete series character on $M^{0}$ for $\lambda_{t}$ and $\mathscr{C}_{t}$ defined as in (9.2). Note that we can choose an ordering $\Phi^{+}$for $\Phi$ so that $\langle\alpha, \beta\rangle \geq 0$ for all $\alpha \in \Phi^{+}$. Set $\Phi_{M}^{+}=\left\{c \alpha: \alpha \in \Phi^{+},\langle\alpha, \beta\rangle=0\right\}$. Then $\left.\rho\right|_{\mathfrak{t}}=\rho_{M}$, where $\rho$ is the half-sum over $\Phi^{+}$and $\rho_{M}$ is the half-sum over $\Phi_{M}^{+}$. Thus $\lambda-\left.\rho\right|_{\mathrm{t}}=\lambda_{\mathrm{t}}-\rho_{M}$. Now since $T \subseteq B, \lambda_{\mathrm{t}} \in \Lambda_{M}=\left\{\lambda \in i \mathrm{t}^{*}: \lambda-\rho_{M}\right.$ is integral $\}$ if $\lambda \in \Lambda$. Further, $Z_{M}\left(M^{0}\right) \subseteq T \subseteq B$ so we can define $\chi \in Z_{M}\left(M^{0}\right)^{\wedge}$ by

$$
\chi=\left.e^{\lambda-\rho}\right|_{Z_{M}\left(M^{0}\right)} .
$$

Now by the above, $\left.\chi\right|_{Z_{M}^{0}}=\left.e^{\lambda_{t}-\rho_{M}}\right|_{Z_{M^{0}}}$ so $\chi \otimes \Theta\left(M^{0}: \lambda_{t}: \mathscr{C}_{t}\right)$ defines a character of $M^{\dagger}=Z_{M}\left(M^{0}\right) M^{0}$. For any $\nu \in \mathfrak{a}_{\mathrm{C}}^{*}$, let

$$
\Theta\left(P: \chi: \lambda_{\mathrm{t}}: \mathscr{C}_{\mathrm{t}}: \nu\right)=\operatorname{Ind}_{M^{\dagger} A N}^{G}\left(\chi \otimes \Theta\left(M^{0}: \lambda_{\mathrm{t}}: \mathscr{C}_{\mathrm{t}}\right) \otimes e^{i \nu} \otimes 1\right) .
$$

Finally, define $\nu(\lambda) \in \mathfrak{a}_{\mathrm{C}}^{*}$ by

$$
\nu(\lambda)=-\left.i^{c} \lambda\right|_{\mathfrak{a}_{\mathbf{c}}}, \quad \lambda \in \Lambda \subseteq \mathfrak{b}_{\mathbf{C}}^{*} .
$$

Now Schmid's identity (when $[G, G]$ has finite center) is

$$
\Theta(\lambda: \mathscr{C})+\Theta\left(\lambda: s_{\beta} \mathscr{C}\right)=\Theta\left(P: \chi: \lambda_{t}: \mathscr{C}_{t}: \nu(\lambda)\right), \quad \text { all } \lambda \in \Lambda[12]
$$

We will generalize this identity to include derivatives along continuous families of relative discrete series parameters. In order to have nontrivial derivatives 
we assume that $\mathscr{H}_{\beta} \cap i \mathfrak{v}^{*} \neq i \mathfrak{v}^{*}$. Then there will be elements $h \in i \mathfrak{v}^{*}$ with $h\left(H_{\beta}^{*}\right) \neq 0$. Fix any $h_{1} \in i \mathfrak{v}^{*}$ with

$$
h_{1}\left(H_{\beta}^{*}\right)=1 \text {. }
$$

Then for any smooth function $\varphi$ of $h \in i v^{*}$ we can define the directional derivative

$$
D_{h}^{\beta} \varphi(h)=\left.\frac{d}{d \xi}\right|_{\xi=0} \varphi\left(h+\xi h_{1}\right) .
$$

Pick $\nu_{1} \in \mathfrak{a}^{*}$ so that

$$
\left\langle\nu_{1},{ }^{c} H_{\beta}^{*}\right\rangle=1 \text {. }
$$

Then for any smooth function $\psi$ of $\nu \in \mathfrak{a}^{*}$ we define

$$
D_{\nu}^{\beta} \psi(\nu)=\left.\frac{d}{d \xi}\right|_{\xi=0} \psi\left(\nu+\xi \nu_{1}\right) \text {. }
$$

Now let $\lambda_{0} \in \Lambda$ and let $\lambda(h)=\lambda_{0}+h, h \in i v^{*}$, be the corresponding continuous family of relative discrete series parameters for $G$. Define $\lambda_{t}(h)=$ $\left.\lambda(h)\right|_{\mathfrak{t}}$ and $\chi(h)=\left.e^{\lambda(h)-\rho}\right|_{Z_{M}\left(M^{0}\right)}$ as in (9.3). Note $\Theta(\lambda(h): \mathscr{C})+\Theta\left(\lambda(h): s_{\beta} \mathscr{C}\right)$ is a smooth function of $h \in i v^{*}$ and $\Theta\left(P: \chi(h): \lambda_{\mathfrak{t}}(h): \mathscr{C}_{t}: \nu\right)$ is a smooth function of $(h, \nu) \in i \mathfrak{v}^{*} \times \mathfrak{a}_{\mathrm{C}}^{*}$.

Theorem 9.6. For any integer $k \geq 0$,

$$
\left(D_{h}^{\beta}\right)^{k}\left(\theta(\lambda(h): \mathscr{C})+\Theta\left(\lambda(h): s_{\beta} \mathscr{C}\right)\right)=\left(D_{h}^{\beta} \pm i D_{\nu}^{\beta}\right)^{k} \Theta\left(P: \chi(h): \lambda_{\mathfrak{t}}(h): \mathscr{C}_{t}: 0\right)
$$

for all $h \in i^{*}$ with $\langle\lambda(h), \beta\rangle=0$.

Proof. Write

$$
F_{1}(h)=\Theta(\lambda(h): \mathscr{C})+\Theta\left(\lambda(h): s_{\beta} \mathscr{C}\right)
$$

and

$$
G(h: \nu)=\Theta\left(P: \chi(h): \lambda_{t}(h): \mathscr{C}_{t}: \nu\right) .
$$

Note that $G(h: \nu)=G(h:-\nu)$ for all $\nu \in \mathfrak{a}_{\mathbf{C}}^{*}$. As in [8, 3.2], $F_{1}$ and $G$ extend to holomorphic functions on $\mathfrak{v}_{\mathbf{C}}^{*}$ and $\mathfrak{v}_{\mathbf{C}}^{*} \times \mathfrak{a}_{\mathbf{C}}^{*}$ respectively. For $h \in \mathfrak{v}_{\mathbf{C}}^{*}$, define $\nu(h)=-\left.i^{c} \lambda(h)\right|_{\mathfrak{a}_{\mathrm{c}}}=-i\left\langle\lambda(h), H_{\beta}^{*}\right\rangle \cdot \nu_{1}$, where $\nu_{1}$ is defined as in (9.5c). Set $F_{2}(h)=G(h: \nu(h)) . F_{2}$ is also a holomorphic function on $\mathfrak{v}_{\mathrm{C}}^{*}$. Schmid's identity (9.4) says that $F_{1}(h)=F_{2}(h)$ for all $h \in i v^{*}$ such that $\lambda(h)$ is a discrete series parameter for a quotient $\bar{G}$ of $G$ with $[\bar{G}, \bar{G}]$ having finite center. Such points are dense in $i \mathfrak{v}^{*}$ so $F_{1}(h)=F_{2}(h)$ for all $h \in \mathfrak{v}_{\mathrm{C}}^{*}$. Thus for any $k \geq 0,\left(D_{h}^{\beta}\right)^{k} F_{1}(h)=\left(D_{h}^{\beta}\right)^{k} F_{2}(h)$ for all $h \in \mathfrak{v}_{\mathrm{C}}^{*}$. But for $h \in \mathfrak{v}_{\mathrm{C}}^{*}$ with $\langle\lambda(h), \beta\rangle=0, F_{2}\left(h+\xi h_{1}\right)=G\left(h+\xi h_{1},-i \xi \nu_{1}\right)$, since $\left\langle\lambda\left(h+\xi h_{1}\right), H_{\beta}^{*}\right\rangle=\xi$. Thus $\left(D_{h}^{\beta}\right)^{k} F_{2}(h)=\left(D_{h}^{\beta}-i D_{\nu}^{\beta}\right)^{k} G(h: 0)$ for all $h$ with $\langle\lambda(h), \beta\rangle=0$. Thus $\left(D_{h}^{\beta}\right)^{k} F_{1}(h)=\left(D_{h}^{\beta}-i D_{\nu}^{\beta}\right)^{k} G(h: 0)$ if $\langle\lambda(h), \beta\rangle=0$. Now since $G(h: \nu)$ is even as a function of $\nu,\left(D_{h}^{\beta}+i D_{\nu}^{\beta}\right)^{k} G(h: 0)=\left(D_{h}^{\beta}-i D_{\nu}^{\beta}\right)^{k} G(h: 0)$. Q.E.D. 


\section{Matching conditions and the Plancherel function}

We return to the notation of $\S \S 4-8$. Fix $\alpha_{1}, \ldots, \alpha_{k} \in \mathscr{C}(\mathscr{D} \times \mathscr{F}), F_{1}, \ldots$, $F_{k} \in \mathscr{S}\left(M^{\dagger}: W\right)$, and write

$$
\begin{gathered}
\Phi(h: \nu: x)=\sum_{i=1}^{k} \alpha_{i}(h: \nu) E\left(P: F_{i}: h: \nu: x\right), \\
\Phi(x)=\int_{\mathscr{D} \times \mathscr{F}} \Phi(h: \nu: x) m(h: \nu) d h d \nu .
\end{gathered}
$$

The main results of $[4,9]$ say that $\Phi \in \mathscr{C}_{H}(G: W)$ if

$$
(h, \nu) \mapsto \Phi(h: \nu: x) m(h: \nu)
$$

is jointly smooth on $\operatorname{cl}(\mathscr{D}) \times \mathscr{F}$.

In Theorem 10.22 we will prove a matching condition for the $\widehat{F}(h: \nu)$, $f \in \mathscr{C}(G)$. As a consequence, we prove in Theorem 10.24 that for $f \in \mathscr{C}_{H}(G)$, $(h, \nu) \mapsto \widehat{F}(h: \nu: x) m(h: \nu)$ is jointly smooth. Thus using Theorem 8.3 , the wave packets

$$
F(H: x)=\int_{\mathscr{D} \times \mathscr{F}} \hat{F}(h: \nu: x) m(h: \nu) d h d \nu
$$

associated to $f \in \mathscr{C}_{H}(G)$ are Schwartz.

We first review the explicit formulas for the Plancherel functions $m(h: \nu)$. Let $\Phi^{+}$denote a set of positive roots for $\Phi=\Phi\left(\mathfrak{g}_{\mathrm{C}}, \mathfrak{h}_{\mathbf{C}}\right)$, and let $\Phi_{R}^{+}=\{\alpha \in$ $\Phi^{+}: \alpha$ takes real values on $\left.\mathfrak{h}\right\}$. For $\alpha \in \Phi_{R}^{+}$, let $H_{\alpha}^{*} \in \mathfrak{a}$ be dual to $\alpha^{\vee}=$ $2 \alpha /\langle\alpha, \alpha\rangle$ under the killing form. Let $X_{\alpha}, Y_{\alpha}$ be elements of the root spaces of $\mathfrak{g}_{\alpha}, \mathfrak{g}_{-\alpha}$ respectively, so that $\theta\left(X_{\alpha}\right)=Y_{\alpha}$ and $\left[X_{\alpha}, Y_{\alpha}\right]=H_{\alpha}^{*}$. Write $Z_{\alpha}=X_{\alpha}-$ $Y_{\alpha}$ and set $\gamma_{\alpha}=\exp \pi Z_{\alpha}$. Then $\gamma_{\alpha} \in Z_{M}\left(M^{0}\right)$. For $\nu \in \mathscr{F}$, write $\nu_{\alpha}=\nu\left(H_{\alpha}^{*}\right)$. Let $\Phi_{\alpha}^{+}=\left\{\beta \in \Phi^{+}:\left.\beta\right|_{a}=c \alpha\right.$ for $\left.c \neq 0\right\}$ and define $\rho_{\alpha}=\frac{1}{2} \sum_{\beta \in \Phi_{\alpha}^{+}} \beta\left(H_{\alpha}^{*}\right)$. Let $\Phi_{R}^{*}=\bigcup_{\alpha \in \Phi_{R}^{+}} \Phi_{\alpha}^{+}$. Finally for $h \in i v^{*}$, we write $\lambda(h)=\lambda_{0}+h_{M}(h)$ and $\chi(h)=\chi_{0} \otimes e^{h}$. Then for $h \in i v^{*}, \nu \in \mathscr{F}$, we define

$$
\varepsilon_{\alpha}(h)=\frac{(-1)^{\rho_{\alpha}} \operatorname{trace}\left[\chi\left(h: \gamma_{\alpha}\right)+\chi\left(h: \gamma_{\alpha}^{-1}\right)\right]}{2 \operatorname{deg} \chi(h)}, \quad \alpha \in \Phi_{R}^{+},
$$

$$
\begin{gathered}
m_{\alpha}^{*}(h: \nu)=\prod_{\beta \in \Phi_{\alpha}^{+}}\langle\lambda(h)+i \nu, \beta\rangle \cdot \frac{\sinh \pi \nu_{\alpha}}{\cosh \pi \nu_{\alpha}-\varepsilon_{\alpha}(h)}, \quad \alpha \in \Phi_{R}^{+}, \\
m_{R}^{*}(h: \nu)=\prod_{\alpha \in \Phi_{R}^{+}} m_{\alpha}^{*}(h: \nu), \\
m(h: \nu)=\prod_{\alpha \in \Phi^{+} \backslash \Phi_{R}^{*}}\langle\lambda(h)+i \nu, \alpha\rangle \cdot m_{R}^{*}(h: \nu) .
\end{gathered}
$$


Lemma 10.3. Let $\alpha \in \Phi_{R}^{+}$. If $\gamma_{\alpha}$ has finite order, then $m_{\alpha}^{*}$ is jointly smooth on iv ${ }^{*} \times \mathscr{F}$. If $\gamma_{\alpha}$ has infinite order, $m_{\alpha}^{*}$ is jointly smooth except possibly at points $\left(h_{0}, \nu_{0}\right)$, where $\nu_{0}\left(H_{\alpha}^{*}\right)=0$ and $\varepsilon_{\alpha}\left(h_{0}\right)=1$.

Proof. Suppose $\gamma_{\alpha}$ has finite order. Since $\chi(h)$ is a unitary character and $\chi\left(h: \gamma_{\alpha}\right)$ depends continuously on $h, \chi\left(h: \gamma_{\alpha}\right)$ and hence $\varepsilon_{\alpha}(h)$ must be independent of $h \in i \mathrm{v}^{*}$.

Now in either case, $m_{\alpha}^{*}$ is jointly smooth except possibly at points $\left(h_{0}, \nu_{0}\right)$ with $\nu_{0}\left(H_{\alpha}^{*}\right)=0$ and $\varepsilon_{\alpha}\left(h_{0}\right)=1$, when the denominator is zero. But if $\gamma_{\alpha}$ has finite order, then $\varepsilon_{\alpha}(h) \equiv 1$, so

$$
m_{\alpha}^{*}(h: \nu)=\prod_{\beta \in \Phi_{\alpha}^{+} \backslash\{\alpha\}}\langle\lambda(h)+i \nu, \beta\rangle \cdot \frac{\nu_{\alpha} \sinh \pi \nu_{\alpha}}{\cosh \pi \nu_{\alpha}-1}
$$

is smooth. Q.E.D.

Fix $\alpha \in \Phi_{R}^{+}$with $\gamma_{\alpha}$ of infinite order. Then $\alpha$ is a root of a simple factor $\mathfrak{g}_{1}$ of $\mathfrak{g}$ with $G_{1}$ simply connected, noncompact, and of hermitian type. From [5, $\S 1$ ] we know that $\Phi_{R}\left(\mathfrak{g}_{1}\right)$, the set of all real roots coming from $\mathfrak{g}_{1}$, is of type $A_{1}^{r} \times C_{s}$ for some $r \geq 0, s \geq 0$, and if $\alpha$ is in the $C_{s}$ factor it is long. Let $\mathfrak{b}$ be a fundamental Cartan subalgebra of $\mathfrak{g}$ and let $\operatorname{SOS}(H)$ denote the set of strongly orthogonal singular imaginary roots of $(\mathfrak{g}, \mathfrak{b})$ used to define the Cayley transform $c$ with $c\left(\mathfrak{b}_{\mathbf{C}}\right)=\mathfrak{h}_{\mathbf{C}}$. Then $c^{-1} \alpha \in \operatorname{SOS}(H)$ and $Z_{\alpha}=c^{-1}\left(i H_{\alpha}^{*}\right) \in \mathfrak{b}$. Set $h_{\alpha}=-i h\left(Z_{\alpha}\right) \in \mathbf{R}$.

Fix $\left(h_{0}, \nu_{0}\right) \in i \mathfrak{v}^{*} \times \mathscr{F}$ so that $\varepsilon_{\alpha}\left(h_{0}\right)=1$ and $\nu_{0}\left(H_{\alpha}^{*}\right)=0$. Then $\chi\left(h_{0}+h\right)\left(\gamma_{\alpha}\right)=e^{\pi i h^{\prime}} \chi_{0}\left(\gamma_{\alpha}\right)$ and trace $\chi\left(h_{0}: \gamma_{\alpha}\right)=\operatorname{trace} \chi\left(h_{0}: \gamma_{\alpha}^{-1}\right)=\operatorname{deg} \chi\left(h_{0}\right)$. $(-1)^{\rho_{\alpha}}$, so that

$$
\varepsilon_{\alpha}\left(h_{0}+h\right)=\cos \pi h_{\alpha}
$$

and

$$
m_{\alpha}^{*}\left(h_{0}+h: \nu_{0}+\nu\right)=\prod_{\beta \in \Phi_{n}^{+}}\left\langle\lambda\left(h_{0}+h\right)+i \nu, \beta\right\rangle \cdot \frac{\sinh \pi \nu_{\alpha}}{\cosh \pi \nu_{\alpha}-\cos \pi h_{\alpha}} .
$$

Thus we have the following.

Lemma 10.5. $(h, \nu) \rightarrow m_{\alpha}^{*}\left(h_{0}+h: \nu_{0}+\nu\right)\left(\nu_{\alpha}^{2}+h_{\alpha}^{2}\right)$ is jointly smooth at $(0,0)$.

For $n \geq 0$, let $(x, y, z)$ denote coordinates in $\mathbf{R}^{n+2}$, where $x, y \in \mathbf{R}$, $z \in \mathbf{R}^{n}$.

Lemma 10.6. Suppose $f \in C^{\infty}\left(\mathbf{R}^{2+n}\right)$ satisfies $D f(0,0, z)=0$ for all $z \in \mathbf{R}^{n}$ and all constant coefficient differential operators $D$. Then

$$
f(x, y, z)\left(x^{2}+y^{2}\right)^{-1} \in C^{\infty}\left(\mathbf{R}^{2+n}\right) .
$$

Proof. For each $z \in \mathbf{R}^{n}$, expand $f$ in a Taylor series in $x$ and $y$ about $(0,0, z)$. Since all derivatives are zero, for each $k$, we just have $f(x, y, z)=$ $R_{k}(x, y, z)$, the $k$ th remainder term. Fix a compact subset $\Omega$ of $\mathbf{R}^{n}$. Then 
for every $k$, there is a constant $C_{k}$ so that $|f(x, y, z)| \leq C_{k}\left(x^{2}+y^{2}\right)^{k}$ for $\left(x^{2}+y^{2}\right) \leq 1, z \in \Omega$. Further, for any $D, D f$ satisfies the same condition as $f$, so there are constants $C_{k, D}$ so that $|D f(x, y, z)| \leq C_{k, D}\left(x^{2}+y^{2}\right)^{k}$ for $x^{2}+y^{2} \leq 1, z \in \Omega$. Let $g(x, y, z)=f(x, y, z)\left(x^{2}+y^{2}\right)^{-1}$. From the above estimates it is easy to see inductively that $g$ has continuous derivatives of all orders also satisfying $D g(0,0, z)=0$ for all $D, z$. Q.E.D.

Lemma 10.7. Suppose $f \in C^{\infty}\left(\mathbf{R}^{2+n}\right)$. Then $f(x, y, z)\left(x^{2}+y^{2}\right)^{-1} \in C^{\infty}\left(\mathbf{R}^{2+n}\right)$ if and only if $\left(\frac{\partial}{\partial x} \pm i \frac{\partial}{\partial y}\right)^{k} f(0,0, z)=0$ for all $k \geq 0, z \in \mathbf{R}^{n}$.

Proof. $\left.\left(\frac{\partial}{\partial x} \pm i \frac{\partial}{\partial y}\right)^{k}\right|_{x=y=0}\left(x^{2}+y^{2}\right)=0$ for all $k$, so one direction is easy. To prove the converse, we use Mather's division theorem [11] to write

$$
f(x, y, z)=\left(x^{2}+y^{2}\right) f_{1}(x, y, z)+x f_{2}(y, z)+f_{3}(y, z),
$$

where $f_{1} \in C^{\infty}\left(\mathbf{R}^{2+n}\right), f_{2}, f_{3} \in C^{\infty}\left(\mathbf{R}^{1+n}\right)$. Assume $\left(\frac{\partial}{\partial x} \pm i \frac{\partial}{\partial y}\right)^{k} f(0,0, z)=0$ for all $z, k$. Thus for all $k$,

$$
\begin{gathered}
\left.\left(\frac{\partial}{\partial x} \pm i \frac{\partial}{\partial y}\right)^{k}\right|_{x=y=0}\left[\left(x^{2}+y^{2}\right) f_{1}(x, y, z)+x f_{2}(y, z)+f_{3}(y, z)\right] \\
=k\left( \pm i \frac{\partial}{\partial y}\right)^{k-1} f_{2}(0, z)+\left( \pm i \frac{\partial}{\partial y}\right)^{k} f_{3}(0, z)=0 .
\end{gathered}
$$

Combining the equalities for $\left(\frac{\partial}{\partial x}+i \frac{\partial}{\partial y}\right)^{k}$ and $\left(\frac{\partial}{\partial x}-i \frac{\partial}{\partial y}\right)^{k}$ we find that $\left(\frac{\partial}{\partial y}\right)^{k-1} f_{2}(0, z)=\left(\frac{\partial}{\partial y}\right)^{k} f_{3}(0, z)=0$. Thus $g(x, y, z)=x f_{2}(y, z)+f_{3}(y, z)$ satisfies $D g(0,0, z)=0$ for all $z$ and all constant coefficient differential operators $D$, so the result follows from Lemma 10.6. Q.E.D.

Let $a \neq 0 \in \mathbf{R}^{n}, b \neq 0 \in \mathbf{R}^{m}$ and define $p \in C^{\infty}\left(\mathbf{R}^{n} \times \mathbf{R}^{m}\right)$ by $p(x, y)=$ $\langle x, a\rangle^{2}+\langle y, b\rangle^{2}$. Take any $\left(x_{a}, x_{b}\right) \in \mathbf{R}^{n} \times \mathbf{R}^{m}$ with $\left\langle x_{a}, a\right\rangle=\left\langle y_{b}, b\right\rangle=$ 1. Let $D_{x}^{a}, D_{y}^{b}$ denote the directional derivatives in the directions of $x_{a}, y_{b}$ respectively, and set $\mathscr{H}(a, b)=\left\{(x, y) \in \mathbf{R}^{n} \times \mathbf{R}^{m}:\langle x, a\rangle=\langle y, b\rangle=0\right\}=$ $\left\{(x, y) \in \mathbf{R}^{n} \times \mathbf{R}^{m}: p(x, y)=0\right\}$. We can now rephrase Lemma 10.7 as follows.

Lemma 10.8. Let $g \in C^{\infty}\left(\mathbf{R}^{n} \times \mathbf{R}^{m}\right)$. Then $g p^{-1}$ is smooth at $\left(x_{0}, y_{0}\right) \in$ $\mathscr{H}(a, b)$ if and only if $\left(D_{x}^{a} \pm i D_{y}^{b}\right)^{k} g\left(x_{0}, y_{0}\right)=0$ for all $k \geq 0$.

Lemma 10.9. Let $a_{1}, \ldots, a_{r} \in \mathbf{R}^{n}$ be linearly independent and $b_{1}, \ldots, b_{r} \in \mathbf{R}^{m}$ be nonzero. Let $p_{i}(x, y)=\left\langle x, a_{i}\right\rangle^{2}+\left\langle y, b_{i}\right\rangle^{2}$. Suppose $g \in C^{\infty}\left(\mathbf{R}^{n} \times \mathbf{R}^{m}\right)$ such that $g p_{i}^{-1} \in C^{\infty}\left(\mathbf{R}^{n} \times \mathbf{R}^{m}\right)$ for $1 \leq i \leq r$. Then $g \prod_{i=1}^{r} p_{i}^{-1} \in C^{\infty}\left(\mathbf{R}^{n} \times \mathbf{R}^{m}\right)$.

Proof. The lemma is trivial if $r=1$. Assume inductively that it is true for $r-1$, where $r \geq 2$. Thus $g \prod_{i=1}^{r-1} p_{i}^{-1} \in C^{\infty}\left(\mathbf{R}^{n} \times \mathbf{R}^{m}\right)$. Let $\mathscr{H}^{\prime}\left(a_{r}, b_{r}\right)=$ $\left\{(x, y) \in \mathscr{H}\left(a_{r}, b_{r}\right): \prod_{i=1}^{r-1} p_{i}(x, y) \neq 0\right\}$. Since $a_{1}, \ldots, a_{r}$ are linearly independent, $\mathscr{H}^{\prime}\left(a_{r}, b_{r}\right)$ is dense in $\mathscr{H}\left(a_{r}, b_{r}\right)$. But for $(x, y) \in \mathscr{H}^{\prime}\left(a_{r}, b_{r}\right)$, 
$\left(D_{x}^{a_{r}} \pm i D_{y}^{b_{r}}\right)^{k} g(x, y)=0$ for all $k \geq 0$ and $\prod_{i=1}^{r-1} p_{i}^{-1}$ is smooth at $(x, y)$. Thus,

$$
\left(D_{x}^{a_{r}} \pm i D_{y}^{b_{r}}\right)^{k}\left[g(x, y) \prod_{i=1}^{r-1} p_{i}(x, y)^{-1}\right]=0 \text { for all } k \geq 0 .
$$

Now since $\mathscr{H}^{\prime}\left(a_{r}, b_{r}\right)$ is dense in $\mathscr{H}\left(a_{r}, b_{r}\right)$,

$$
\left(D_{x}^{a_{r}} \pm i D_{y}^{b_{r}}\right)^{k}\left[g(x, y) \prod_{i=1}^{r-1} p_{i}(x, y)^{-1}\right]=0 \text { for all } k \geq 0, \quad(x, y) \in \mathscr{H}\left(a_{r}, b_{r}\right) \text {. }
$$

Thus, $g \cdot \prod_{i=1}^{r-1} p_{i}^{-1} p_{r}^{-1}=g \cdot \prod_{i=1}^{r} p_{i}^{-1}$ is smooth by Lemma 10.8. Q.E.D.

Lemma 10.10. Let $D$ be a first-order constant coefficient differential operator on $\mathbf{R}^{n}$, and suppose $g, h \in C^{\infty}\left(\mathbf{R}^{n}\right), x_{0} \in \mathbf{R}^{n}$, such that $D^{k}(g h)\left(x_{0}\right)=0$ for all $k \geq 0$. Then either $D^{k} g\left(x_{0}\right)=0$ for all $k \geq 0$, or $D^{k} h\left(x_{0}\right)=0$ for all $k \geq 0$.

Proof. Suppose there is $m \geq 0$ so that $D^{m} g\left(x_{0}\right) \neq 0$. We will prove that $D^{k} h\left(x_{0}\right)=0$ for all $k \geq 0$. Pick the smallest $m$ with $D^{m} g\left(x_{0}\right) \neq 0$. Then

$$
0=D^{m}(g \cdot h)\left(x_{0}\right)=\sum_{j=0}^{m}\left(\begin{array}{c}
m \\
j
\end{array}\right) D^{j} g\left(x_{0}\right) D^{m-j} h\left(x_{0}\right)=D^{m} g\left(x_{0}\right) h\left(x_{0}\right),
$$

so $h\left(x_{0}\right)=0$. Assume inductively that $D^{j} h\left(x_{0}\right)=0$ for all $0 \leq j \leq r$. Then

$$
0=D^{m+r+1}(g \cdot h)\left(x_{0}\right)=\left(\begin{array}{c}
m+r+1 \\
m
\end{array}\right) D^{m} g\left(x_{0}\right) D^{r+1} h\left(x_{0}\right)
$$

since $D^{j} g\left(x_{0}\right)=0$ for $0 \leq j \leq m-1$ and $D^{m+r+1-j} h\left(x_{0}\right)=0$ for $m+1 \leq$ $j \leq m+r+1$. Thus $D^{r+1} h\left(x_{0}\right)=0$. Q.E.D.

We will apply Lemmas $10.8,10.9$, and 10.10 to the situation of $\mathbf{R}^{n} \times \mathbf{R}^{m}=$ $i v^{*} \times \mathscr{F}$ and $p_{\alpha}(h, \nu)=h\left(-i Z_{\alpha}\right)^{2}+\nu\left(H_{\alpha}^{*}\right)^{2}=h_{\alpha}^{2}+\nu_{\alpha}^{2}$. Fix $\left(h_{1}, \nu_{1}\right) \in i v^{*} \times \mathscr{F}$ so that $h_{1}\left(-i Z_{\alpha}\right)=1=\nu_{1}\left(H_{\alpha}^{*}\right)$, and denote the associated directional derivatives by $D_{h}^{\alpha}, D_{\nu}^{\alpha}$ respectively.

Lemma 10.11. Let $g \in C^{\infty}\left(i v^{*} \times \mathscr{F}\right)$. Then $g \cdot m_{R}^{*} \in C^{\infty}\left(i v^{*} \times \mathscr{F}\right)$ if and only if for any $\alpha$ and $\left(h_{0}, \nu_{0}\right)$ with $m_{\alpha}^{*}$ not jointly smooth at $\left(h_{0}, \nu_{0}\right)$ we have $\left(D_{h}^{\alpha} \pm i D_{\nu}^{\alpha}\right)^{k} g\left(h_{0}: \nu_{0}\right)=0$ for all $k \geq 0$.

Proof. Assume first that $g$ satisfies the derivative condition. Fix $\left(h_{0}, \nu_{0}\right) \in$ $i v^{*} \times \mathscr{F}$ and let $\Phi_{0}=\left\{\alpha \in \Phi_{R}^{+}: m_{\alpha}^{*}\right.$ is not smooth at $\left.\left(h_{0}, \nu_{0}\right)\right\} \subseteq\left\{\alpha \in \Phi_{R}^{+}: \gamma_{\alpha}\right.$ has infinite order, $\varepsilon_{\alpha}\left(h_{0}\right)=1$, and $\left.\nu_{0}\left(H_{\alpha}^{*}\right)=0\right\}$. Note that $c^{-1} \Phi_{0} \subseteq \operatorname{SOS}(H)$ so roots in $\Phi_{0}$ are linearly independent. For $\alpha \in \Phi \backslash \Phi_{0}, m_{\alpha}^{*}$ is smooth at $\left(h_{0}, \nu_{0}\right)$. For $\alpha \in \Phi_{0}, m_{\alpha}^{*}\left(h_{0}+h: \nu_{0}+\nu\right)\left(h_{\alpha}^{2}+\nu_{\alpha}^{2}\right)$ is smooth at $(0,0)$ by Lemma 10.5. Thus $g \cdot m_{R}^{*}$ is smooth at $\left(h_{0}, \nu_{0}\right)$ if $\tilde{g} \cdot p^{-1}$ is smooth at $(0,0)$, where $\tilde{g}(h: \nu)=g\left(h_{0}+h: \nu_{0}+\nu\right)$ and $p(h: \nu)=\prod_{\alpha \in \Phi_{0}} p_{\alpha}(h: \nu)$, $p_{\alpha}(h: \nu)=\nu_{\alpha}^{2}+h_{\alpha}^{2}$. But the derivative hypothesis implies by Lemma 10.8 that 
$\tilde{g} \cdot p_{\alpha}^{-1}$ is smooth at $(0,0)$ for all $\alpha \in \Phi_{0}$. Now Lemma 10.9 implies that $\tilde{g} \cdot p^{-1}$ is smooth at $(0,0)$.

For the converse, assume $g \cdot m_{R}^{*} \in C^{\infty}\left(i \mathfrak{v}^{*} \times \mathscr{F}\right)$. Fix $\alpha$ and $\left(h_{0}, \nu_{0}\right)$ with $m_{\alpha}^{*}$ not jointly smooth at $\left(h_{0}, \nu_{0}\right)$. Write $D=D_{\alpha}^{h} \pm i D_{\alpha}^{\nu}$, and define $\Phi_{0}=\left\{\beta \in \Phi_{R}^{+}: m_{\beta}^{*}\right.$ is not smooth at $\left.\left(h_{0}, \nu_{0}\right)\right\}$. Then

$$
\prod_{\beta \in \Phi_{0} \backslash\{\alpha\}}\left(h_{\beta}^{2}+\nu_{\beta}^{2}\right) \cdot g \cdot m_{R}^{*}=\left[g \cdot \prod_{\beta \in \Phi_{R}^{+} \backslash \Phi_{0}} m_{\beta}^{*} \cdot \prod_{\beta \in \Phi_{0}} m_{\beta}^{*}\left(h_{\beta}^{2}+\nu_{\beta}^{2}\right)\right] /\left(h_{\alpha}^{2}+\nu_{\alpha}^{2}\right)
$$

is smooth by hypothesis, so

$$
D^{k}\left[g \cdot \prod_{\beta \in \Phi_{R}^{+} \backslash \Phi_{0}} m_{\beta}^{*} \cdot \prod_{\beta \in \Phi_{0}} m_{\beta}^{*}\left(h_{\beta}^{2}+\nu_{\beta}^{2}\right)\right]=0
$$

for all $k \geq 0$ by Lemma 10.8. But $g, m_{\beta}^{*}, \beta \in \Phi_{R}^{+} \backslash \Phi_{0}$, and $m_{\beta}^{*}\left(h_{\beta}^{2}+\nu_{\beta}^{2}\right), \beta \in$ $\Phi_{0}$, are all smooth. Thus by Lemma 10.10 , one of these functions must have $D^{k}=0$ at $\left(h_{0}, \nu_{0}\right)$ for all $k \geq 0$. Now this is not true of $m_{\beta}^{*}, \beta \in \Phi_{R}^{+} \backslash \Phi_{0}$, or $m_{\beta}^{*}\left(h_{\beta}^{2}+\nu_{\beta}^{2}\right), \beta \in \Phi_{0}$. Thus $D^{k} g\left(h_{0}, \nu_{0}\right)=0$ for all $k \geq 0$. Q.E.D.

Theorem 10.12. $\widehat{F}(h: \nu: x) m_{R}^{*}(h: \nu)$ is jointly smooth on $\mathrm{cl}(\mathscr{D}) \times \mathscr{F}$ if and only if for every $\alpha \in \Phi_{R}^{+}$and every $\left(h_{0}, \nu_{0}\right) \in \mathscr{D} \times \mathscr{F}$ with $m_{\alpha}^{*}$ not jointly smooth at $\left(h_{0}, \nu_{0}\right)$ we have $\left(D_{h}^{\alpha} \pm i D_{\nu}^{\alpha}\right)^{k} \widehat{F}\left(h_{0}: \nu_{0}: x\right)=0$ for all $x \in G$, $k \geq 0$.

Proof. By Lemma 10.11 it suffices to show that $\left(D_{h}^{\alpha} \pm i D_{\nu}^{\alpha}\right)^{k} \widehat{F}\left(h_{0}: \nu_{0}: x\right) \equiv 0$ for all $\left(h_{0}, \nu_{0}\right) \in(\mathrm{cl} \mathscr{D} \backslash \mathscr{D}) \times \mathscr{F}$. But by $(10.1)$,

$$
\widehat{F}(h: \nu: x)=\sum_{i} \alpha_{i}(h: \nu) E\left(P: F_{i}: h: \nu: x\right),
$$

where the $E\left(P: F_{i}: h: \nu: x\right)$ are jointly smooth for $(h, \nu) \in i v^{*} \times \mathscr{F}$ and the $\alpha_{i} \in \mathscr{C}(\mathscr{D} \times \mathscr{F})$. But for every $\alpha \in \mathscr{C}(\mathscr{D} \times \mathscr{F}), D \in D\left(i v^{*} \times \mathscr{F}\right)$, and $h_{0} \in \operatorname{cl}(\mathscr{D}) \backslash \mathscr{D}, D \alpha\left(h_{0}: \nu\right)=0$ for all $\nu \in \mathscr{F}$. Thus for all $D \in D\left(i v^{*} \times \mathscr{F}\right)$ and $h_{0} \in \operatorname{cl}(\mathscr{D}) \backslash \mathscr{D}, D \widehat{F}\left(h_{0}: \nu: x\right)=0$ for all $\nu \in \mathscr{F}, x \in G$. Q.E.D.

Fix a root $\alpha \in \Phi_{R}^{+}$with $\gamma_{\alpha}$ of infinite order. Recall $c^{-1} \alpha \in \operatorname{SOS}(H)$. Let $H^{\prime}=T^{\prime} \times A^{\prime}$ be the Cartan subgroup of $G$ with $\operatorname{SOS}\left(H^{\prime}\right)=\operatorname{SOS}(H) \backslash c^{-1} \alpha$ used to define the Cayley transform $c^{\prime}$ with $c^{\prime}\left(\mathfrak{b}_{\mathbf{C}}\right)=\mathfrak{h}_{\mathbf{C}}^{\prime}$. Thus, $c=c_{\alpha} c^{\prime}$, where $c_{\alpha}$ is the Cayley transform corresponding to $\alpha$ and $c_{\alpha}\left(\mathfrak{h}^{\prime}\right)_{\mathbf{C}}=\mathfrak{h}_{\mathbf{C}}$. We have $\mathfrak{t} \subseteq \mathfrak{t}^{\prime} \subseteq \mathfrak{b}$ and $\mathfrak{a}^{\prime}=\{H \in \mathfrak{a}: \alpha(H)=0\}$. Write $\alpha^{\prime}=c_{\alpha}^{-1} \alpha \in \Phi\left(\mathfrak{g}, \mathfrak{h}^{\prime}\right)$. Note $Z_{\alpha} \in \mathfrak{t}^{\prime}$ so that $\gamma_{\alpha} \in \exp \left(\mathfrak{t}^{\prime}\right)$ and $-i Z_{\alpha}=c_{\alpha}^{-1}\left(H_{\alpha}^{*}\right)$. Let $P^{\prime}=M^{\prime} A^{\prime} N^{\prime}$ be a parabolic subgroup corresponding to $H^{\prime}$.

Lemma 10.13. Let $h_{0} \in i \mathfrak{v}^{*}$ such that $\varepsilon_{\alpha}\left(h_{0}\right)=1$. Then there is $\lambda^{\prime} \in\left(i \mathfrak{t}^{\prime}\right)^{*}$ such that:

(i) $\lambda^{\prime}-\rho_{M^{\prime}}$ is integral; 
(ii) $\left.\lambda^{\prime}\right|_{\mathfrak{t}}=\lambda_{0}$;

(iii) $\operatorname{tr} \chi_{0}\left(\gamma_{\alpha}\right) / \operatorname{deg} \chi_{0}=e^{\lambda^{\prime}-\rho_{M^{\prime}}}\left(\gamma_{\alpha}\right)$;

(iv) $\varepsilon_{\alpha}(h)=\cos \pi\left\langle\lambda^{\prime}+h_{M^{\prime}}(h),-i Z_{\alpha}\right\rangle$ for all $h \in i v^{*}$;

(v) $\left\langle\lambda^{\prime}+h_{M^{\prime}}\left(h_{0}\right), \alpha^{\prime}\right\rangle=0$.

Proof. We need to define compatible orderings for several root systems. Fix a set $\Sigma^{\prime}$ of positive restricted roots for $\left(\mathfrak{g}, \mathfrak{a}^{\prime}\right)$ and a set $\Phi_{M}^{+}$of positive roots for $\Phi\left(\mathfrak{m}_{\mathbf{C}}, \mathfrak{t}_{\mathbf{C}}\right)$. Now define $\Phi^{+}=\Phi^{+}\left(\mathfrak{g}_{\mathbf{C}}, \mathfrak{h}_{\mathbf{C}}\right)$ by $\Phi^{+}=\left\{\beta \in \Phi:\left.\beta\right|_{\mathfrak{a}^{\prime}} \epsilon\right.$ $\Sigma^{\prime},\left.\beta\right|_{\mathfrak{a}^{\prime}}=0$ and $\langle\beta, \alpha\rangle>0$, or $\left.\beta\right|_{\mathfrak{a}}=0$ and $\left.\beta \in \Phi_{M}^{+}\right\}$. Let $\Phi_{M^{\prime}}^{+}=$ $c_{\alpha}^{-1}\left\{\beta \in \Phi^{+}:\left.\beta\right|_{\mathfrak{a}^{\prime}}=0\right\}$. Let $\Phi_{\mathfrak{b}}^{+}=c^{-1} \Phi^{+}$. Define $\rho_{G}, \rho_{M}, \rho_{M^{\prime}}$ to be the half-sums of roots in $\Phi_{\mathfrak{b}}^{+}, \Phi_{M}^{+}$, and $\Phi_{M^{\prime}}^{+}$, respectively. With these orderings, $\left.\rho_{G}\right|_{\mathfrak{t}}=\rho_{M},\left.\rho_{G}\right|_{\mathfrak{t}^{\prime}}=\rho_{M^{\prime}},\left.\rho_{M^{\prime}}\right|_{\mathfrak{t}}=\rho_{M}$, and $(-1)^{\rho_{\alpha}}=e^{\rho_{M^{\prime}}}\left(\gamma_{\alpha}\right)$.

Write $\mathfrak{g}=\mathfrak{g}_{1} \oplus \mathfrak{g}_{2}$, where $\mathfrak{g}_{1}$ is the simple ideal of $\mathfrak{g}$ with $\alpha$ as a root and $\mathfrak{g}_{2}$ is its orthocomplement. If $\mathfrak{s}$ is any subalgebra of $\mathfrak{g}$, we write $\mathfrak{s}_{i}=\mathfrak{s} \cap \mathfrak{g}_{i}$, $i=1,2$. Then we have $t_{1} \subseteq \mathfrak{t}_{1}^{\prime} \subseteq \mathfrak{b}_{1}$ and $t_{2}=\mathfrak{t}_{2}^{\prime}$. In fact, since $\mathfrak{g}_{1}$ is of hermitian type we have $T_{1} \subseteq B_{1}=B_{1}^{0}$. In particular, $T_{1}$ is abelian. Let $\lambda_{0,1}=$ $\left.\lambda_{0}\right|_{\mathfrak{t}_{1}}, \rho_{M, 1}=\left.\rho_{M}\right|_{\mathfrak{t}_{1}}$, and $\chi_{0,1} \in Z_{M_{1}}\left(M_{1}^{0}\right)^{\wedge}$ so that $\left.\chi_{0}\right|_{Z_{M_{1}}\left(M_{1}^{0}\right)}$ is a multiple of $\chi_{0,1}$. Then $\chi_{0,1} \otimes e^{\lambda_{0,1}-\rho_{M, 1}}$ is a character of $T_{1}$, hence the restriction of a character of $B_{1}=\exp \left(\mathfrak{b}_{1}\right)$. Thus, there is $\lambda_{G_{1}} \in i \mathfrak{b}_{1}^{*}$ so that $e^{\lambda_{G_{1}}-\rho_{G_{1}}}$ is well defined on $B_{1}$ and $\left.e^{\lambda_{G_{1}}-\rho_{G_{1}}}\right|_{T_{1}}=\chi_{0,1} \otimes e^{\lambda_{0,1}-\rho_{M, 1}}$. Here $\rho_{G_{1}}=\left.\rho_{G}\right|_{\mathfrak{b}_{1}}$. We can assume $\lambda_{G_{1}}$ is chosen so that $\left.\lambda_{G_{1}}\right|_{t_{1}}=\lambda_{0,1}$. Define $\lambda^{\prime} \in\left(i \mathrm{t}^{\prime}\right)^{*}$ by $\left.\lambda^{\prime}\right|_{\mathfrak{t}_{2}^{\prime}}=\left.\lambda_{0}\right|_{\mathfrak{t}_{2}}$ and $\left.\lambda^{\prime}\right|_{\mathfrak{t}_{1}^{\prime}}=\left.\lambda_{G_{1}}\right|_{\mathfrak{t}_{1}^{\prime}}$. By construction $\lambda^{\prime}$ satisfies (ii). Now $e^{\lambda^{\prime}-\rho_{M^{\prime}}}$ is well defined on $\exp \left(\mathfrak{t}_{2}^{\prime}\right)$ since $\exp \left(\mathfrak{t}_{2}^{\prime}\right)=\exp \left(\mathfrak{t}_{2}\right)$ and $\lambda^{\prime}-\left.\rho_{M^{\prime}}\right|_{\mathfrak{t}_{2}^{\prime}}=\lambda_{0}-\left.\rho_{M}\right|_{\mathfrak{t}_{2}^{\prime}}$. Also $e^{\lambda^{\prime}-\rho_{M^{\prime}}}$ is well defined on $\exp \left(\mathfrak{t}_{1}^{\prime}\right)$ since $\lambda^{\prime}-\left.\rho_{M^{\prime}}\right|_{\mathfrak{t}_{1}^{\prime}}=\lambda_{G_{1}}-\left.\rho_{G_{1}}\right|_{\mathfrak{t}_{1}^{\prime}}$. Finally, $\exp \left(\mathfrak{t}_{1}^{\prime}\right) \cap \exp \left(\mathfrak{t}_{2}^{\prime}\right) \subseteq Z_{M}\left(M^{0}\right) \cap T^{0}$ and

$$
\left.e^{\lambda_{0}-\rho_{M}}\right|_{\left(T_{1}^{\prime}\right)^{0} \cap\left(T_{2}^{\prime}\right)^{0}}=\left.\chi_{0}\right|_{\left(T_{1}^{\prime}\right)^{0} \cap\left(T_{2}^{\prime}\right)^{0}}=\left.e^{\lambda_{G_{1}}-\rho_{G_{1}}}\right|_{\left(T_{1}^{\prime}\right)^{0} \cap\left(T_{2}^{\prime}\right)^{0}} \text {. }
$$

Thus $\lambda^{\prime}$ satisfies $(i)$.

Now $\gamma_{\alpha} \in Z_{M_{1}}\left(M_{1}^{0}\right) \cap \exp \left(\mathfrak{t}_{1}^{\prime}\right)$ and $\chi_{0,1}\left(\gamma_{\alpha}\right)=e^{\lambda_{G_{1}}-\rho_{G_{1}}}\left(\gamma_{\alpha}\right)=e^{\lambda^{\prime}-\rho_{M^{\prime}}}\left(\gamma_{\alpha}\right)$, so $\lambda^{\prime}$ satisfies (iii). In particular, trace $\chi\left(h: \gamma_{\alpha}\right) / \operatorname{deg} \chi_{0}=e^{\lambda^{\prime}+h_{M^{\prime}}(h)-\rho_{M^{\prime}}}\left(\gamma_{\alpha}\right)$ for all $h \in i \mathrm{v}^{*}$. Thus $\varepsilon_{\alpha}(h)=\cos \pi\left\langle\lambda^{\prime}+h_{M^{\prime}}(h),-i Z_{\alpha}\right\rangle$ for all $h \in i \mathrm{v}^{*}$. Now if $\varepsilon_{\alpha}\left(h_{0}\right)=1,\left\langle\lambda^{\prime}+h_{M^{\prime}}\left(h_{0}\right),-i Z_{\alpha}\right\rangle=\left\langle\lambda^{\prime}+h_{M^{\prime}}\left(h_{0}\right),\left(\alpha^{\prime}\right)^{v}\right\rangle=2 m_{0}$ for some $m_{0} \in Z$. If $m_{0} \neq 0$, we can replace $\lambda^{\prime}$ by $\lambda^{\prime}-m_{0} \alpha^{\prime}$. Properties (i)-(iv) are preserved, and $(\mathrm{v})$ is now satisfied. Q.E.D.

Lemma 10.14. $Z_{M}\left(M^{0}\right)=Z_{M^{\prime}}\left(M^{\prime 0}\right) Z_{M \cap M^{\prime 0}}\left(M^{0}\right)$. Further, $Z_{M \cap M^{\prime 0}}\left(M^{0}\right)$ is contained in the center of $Z_{M}\left(M^{0}\right)$.

Proof. We always have $Z_{M^{\prime}}\left(M^{\prime 0}\right) Z_{M \cap M^{\prime 0}}\left(M^{0}\right) \subseteq Z_{M}\left(M^{0}\right)$, and

$$
\left[Z_{M}\left(M^{0}\right) / Z_{M^{\prime}}\left(M^{\prime 0}\right) Z_{M \cap M^{\prime 0}}\left(M^{0}\right)\right]=\left[T / T \cap T^{\prime}\right] .
$$


Thus for the first part it suffices to show that $T \subseteq T^{\prime}$. Write $\mathfrak{g}=\mathfrak{g}_{1} \oplus \mathfrak{g}_{2}$ as in the proof of Lemma 10.13. Since $T_{2}=T_{2}^{\prime}$ it is enough to show that $T_{1} \subseteq T_{1}^{\prime}$. But here $T_{1}=\left\{b \in B_{1}: e^{\alpha}(b)=1\right.$ for all $\left.\alpha \in \operatorname{SOS}(H)\right\} \subseteq T_{1}^{\prime}=\left\{b \in B_{1}: e^{\alpha}(b)=1\right.$ for all $\left.\alpha \in \operatorname{SOS}\left(H^{\prime}\right)\right\}$ since $\operatorname{SOS}\left(H^{\prime}\right) \subseteq \operatorname{SOS}(H)$.

Now decompose $Z_{M \cap M^{\prime 0}}\left(M^{0}\right)=Z_{M_{1} \cap M_{1}^{\prime 0}}\left(M_{1}^{0}\right) Z_{M_{2} \cap M_{2}^{\prime 0}}\left(M_{2}^{0}\right)$. Since $M_{2}=$ $M_{2}^{\prime}$ we have $Z_{M_{2} \cap M_{2}^{\prime 0}}\left(M_{2}^{0}\right)=Z_{M_{2}^{0}}$, the center of $M_{2}^{0}$. This is central in $Z_{M}\left(M^{0}\right)$. But $Z_{M_{1} \cap M_{1}^{\prime 0}}\left(M_{1}^{0}\right) \subseteq Z_{M_{1}}\left(M_{1}^{0}\right)$ which is abelian since $\mathfrak{g}_{1}$ is of hermitian type, and hence is central in $Z_{M}\left(M^{0}\right)$. Q.E.D.

Let ${ }^{*} P=P \cap M^{\prime 0}$ and write its Langlands decomposition ${ }^{*} P={ }^{*} M^{*} A^{*} N$, where ${ }^{*} M=M \cap M^{\prime 0}$. Write ${ }^{*} P^{\dagger}={ }^{*} M^{\dagger *} A^{*} N$ where ${ }^{*} M^{\dagger}=Z^{*}{ }_{M}\left(M^{0}\right) M^{0}$. For $\chi_{0} \in Z_{M}\left(M^{0}\right)^{\wedge}$, let $\chi_{0}^{\prime}=\left.\chi_{0}\right|_{Z_{M^{\prime}}\left(M^{\prime 0}\right)}$ and let $\chi_{0}^{*}$ be the character by which $\chi_{0}$ acts on the central subgroup $Z_{M_{M}}\left(M^{0}\right)$. Write $\chi_{0}^{\prime}(h)=\chi_{0}^{\prime} \otimes e^{h}, \chi_{0}^{*}(h)=$ $\chi_{0}^{*} \otimes e^{h}, h \in i 0^{*}$. We can define a family of unitary representations of $M^{\prime 0}$ by (10.15a) $\pi\left(M^{\prime 0}:{ }^{*} P: \chi_{0}^{*}(h): \lambda(h): \nu^{*}\right)$

$$
\begin{aligned}
= & \operatorname{Ind}_{\left.Z{ }^{*} M^{(} M^{0}\right) M^{0 *} A^{*} N}^{M_{N}}\left(\chi_{0}^{*}(h) \otimes \Theta\left(M^{0}: \lambda(h)\right) \otimes e^{i \nu^{*}} \otimes 1\right), \\
& h \in i \mathfrak{v}^{*}, \nu^{*} \in{ }^{*} \mathfrak{a}^{*} .
\end{aligned}
$$

We denote the corresponding characters by

$$
\Theta\left(M^{\prime 0}:{ }^{*} P: \chi_{0}^{*}(h): \lambda(h): \nu^{*}\right) \text {. }
$$

Lemma 10.16. For $\nu \in \mathfrak{a}^{*}$, let $\nu^{\prime}=\left.\nu\right|_{\mathfrak{a}^{\prime}}$ and $\nu^{*}=\left.\nu\right|_{{ }_{\mathfrak{a}}}$. Then

$$
\begin{aligned}
& \pi(H: \chi(h): \lambda(h): \nu) \\
& \quad=\operatorname{Ind}_{Z_{M^{\prime}}\left(M^{\prime 0}\right) M^{\prime 0} A^{\prime} N^{\prime}}\left(\chi_{0}^{\prime}(h) \otimes \pi\left(M^{\prime 0}:{ }^{*} P: \chi_{0}^{*}(h): \lambda(h): \nu^{*}\right) \otimes e^{i \nu^{\prime}} \otimes 1\right) .
\end{aligned}
$$

Proof. Use Lemma 10.14 and induction by stages. Q.E.D.

For any chambers $\mathscr{C}$ of $i \mathrm{t}^{*}$ with respect to $\Phi\left(\mathfrak{m}_{\mathrm{C}}, \mathfrak{t}_{\mathrm{C}}\right)$ and $\mathscr{C}^{\prime}$ of $\left(i \mathrm{t}^{\prime}\right)^{*}$ with respect to $\Phi\left(\mathfrak{m}_{\mathrm{C}}^{\prime}, \mathfrak{t}_{\mathrm{C}}^{\prime}\right)$ we write

$$
\begin{aligned}
& \Theta(H: \chi(h): \lambda(h): \mathscr{C}: \nu) \\
& \quad=\operatorname{Ind}_{M^{\dagger} A N}^{G}\left(\chi(h) \otimes \Theta\left(M^{0}: \lambda(h): \mathscr{C}\right) \otimes e^{i \nu} \otimes 1\right)
\end{aligned}
$$

and

$$
\begin{aligned}
\Theta\left(H^{\prime}: \chi^{\prime}(h): \lambda^{\prime}(h): \mathscr{C}^{\prime}: \nu^{\prime}\right) \\
\quad=\operatorname{Ind}_{\left(M^{\prime}\right)^{\dagger} A^{\prime} N^{\prime}}^{G}\left(\chi^{\prime}(h) \otimes \theta\left(M^{\prime 0}: \lambda^{\prime}(h): \mathscr{C}^{\prime}\right) \otimes e^{i \nu^{\prime}} \otimes 1\right),
\end{aligned}
$$

where $\Theta\left(M^{0}: \lambda(h): \mathscr{C}\right)$ and $\Theta\left(M^{\prime 0}: \lambda^{\prime}(h): \mathscr{C}^{\prime}\right)$ are coherently continued relative discrete series characters defined as in (9.2).

Now let $\mathscr{C}^{\prime}$ be a chamber of $\left(i t^{\prime}\right)^{*}$ so that $\mathscr{C}^{\prime}$ and $s_{\alpha^{\prime}} \mathscr{C}^{\prime}$ are separated only by the root hyperplane $\mathscr{H}_{\alpha^{\prime}}$. Let $\mathscr{C}$ be a chamber of $i t^{*}$ so that $\left.\tau\right|_{\mathfrak{t}} \in \mathscr{C}$ for all $\tau \in \mathscr{C}^{\prime} \cup s_{\alpha^{\prime}} \mathscr{C}^{\prime}$. 
Proposition 10.18. Fix $h_{0} \in i v^{*}$ such that $\varepsilon_{\alpha}\left(h_{0}\right)=1$ and define $\lambda^{\prime} \in\left(i \mathrm{t}^{\prime}\right)^{*}$ as in Lemma 10.13, $\chi_{0}^{\prime}=\left.\chi_{0}\right|_{Z_{\mathcal{M}^{\prime}}\left(M^{\prime 0}\right)}$. Write $\lambda^{\prime}(h)=\lambda^{\prime}+h_{M^{\prime}}(h), h \in i \mathfrak{v}^{*}$. For any $\nu_{0} \in \mathfrak{a}^{*}$ with $\nu_{0}\left(H_{\alpha}^{*}\right)=0$ we set $\nu_{0}^{\prime}=\left.\nu_{0}\right|_{\mathfrak{a}^{\prime}}$. Then for all $k \geq 0$,

$$
\begin{gathered}
\left(D_{h}^{\alpha} \pm i D_{\nu}^{\alpha}\right)^{k}\left[\Theta\left(H^{\prime}: \chi^{\prime}\left(h_{0}\right): \lambda^{\prime}\left(h_{0}\right): \mathscr{C}^{\prime}: \nu_{0}^{\prime}\right)\right. \\
\left.\quad+\Theta\left(H^{\prime}: \chi^{\prime}\left(h_{0}\right): \lambda^{\prime}\left(h_{0}\right): s_{\alpha^{\prime}} \mathscr{C}^{\prime}: \nu_{0}^{\prime}\right)\right] \\
=\left(D_{h}^{\alpha} \pm i D_{\nu}^{\alpha}\right)^{k} \Theta\left(H: \chi\left(h_{0}\right): \lambda\left(h_{0}\right): \mathscr{C}: \nu_{0}\right) .
\end{gathered}
$$

Proof. For all $(h, \nu)$ we have

$$
\begin{aligned}
& \Theta\left(H^{\prime}: \chi^{\prime}(h): \lambda^{\prime}(h): \mathscr{C}^{\prime}: \nu^{\prime}\right)+\Theta\left(H^{\prime}: \chi^{\prime}(h): \lambda^{\prime}(h): s_{\alpha^{\prime}} \mathscr{C}^{\prime}: \nu^{\prime}\right) \\
& =\operatorname{Ind}_{Z_{M^{\prime}}\left(M^{\prime 0}\right) M^{\prime 0} A^{\prime} N^{\prime}}^{G}\left(\chi ^ { \prime } ( h ) \otimes \left[\Theta\left(M^{\prime 0}: \lambda^{\prime}(h): \mathscr{C}^{\prime}\right)\right.\right. \\
& \left.\left.+\Theta\left(M^{\prime 0}: \lambda^{\prime}(h): s_{\alpha^{\prime}} \mathscr{C}^{\prime}\right)\right] \otimes e^{i \nu^{\prime}} \otimes 1\right)
\end{aligned}
$$

and

$$
\begin{aligned}
\Theta(H & : \chi(h): \lambda(h): \mathscr{C}: \nu) \\
& =\operatorname{Ind}_{Z_{M^{\prime}}\left(M^{\prime 0}\right) M^{\prime 0} A^{\prime} N^{\prime}}^{G}\left(\chi^{\prime}(h) \otimes \Theta\left(M^{\prime 0}:{ }^{*} P: \chi^{*}(h): \lambda(h): \mathscr{C}: \nu^{*}\right) \otimes e^{i \nu^{\prime}} \otimes 1\right)
\end{aligned}
$$

by Lemma 10.16. Further, for all $\xi \in \mathbf{R},\left(\nu_{0}+\xi \nu_{1}\right)^{*}=\xi \nu_{1}$. Thus it suffices to prove that

$$
\begin{aligned}
\left(D_{h}^{\alpha}\right)^{k} & {\left[\Theta\left(M^{\prime 0}: \lambda^{\prime}\left(h_{0}\right): \mathscr{C}^{\prime}\right)+\Theta\left(M^{\prime 0}: \lambda^{\prime}\left(h_{0}\right): s_{\alpha^{\prime}} \mathscr{C}^{\prime}\right)\right] } \\
= & \left(D_{h}^{\alpha} \pm i D_{\nu}^{\alpha}\right)^{k} \Theta\left(M^{\prime 0}:{ }^{*} P: \chi^{*}\left(h_{0}\right): \lambda\left(h_{0}\right): \mathscr{C}: 0\right)
\end{aligned}
$$

for all $k \geq 0$. This is Theorem 9.6, since if $\beta=\alpha^{\prime}$, then $-i Z_{\alpha}=H_{\beta}^{*}$ and $H_{\alpha}^{*}=$ $c_{\alpha} H_{\beta}^{*}$ so that $D_{h}^{\alpha}=D_{h}^{\beta}, D_{\nu}^{\alpha}=D_{\nu}^{\beta}$ in the notation of Theorem 9.6. Q.E.D.

Fix $h_{0} \in \mathscr{D}$ with $\varepsilon_{\alpha}\left(h_{0}\right)=1$ and let $\Phi_{0}=\left\{\beta \in \Phi\left(\mathfrak{m}^{\prime}, \mathfrak{t}^{\prime}\right):\left\langle\beta, \lambda^{\prime}\left(h_{0}\right)\right\rangle=0\right\}$, where $\lambda^{\prime}$ is defined as in Lemma 10.13 .

Lemma 10.19. Assume $h_{0} \in \mathscr{D}$. Then either $\Phi_{0}=\left\{ \pm \alpha^{\prime}\right\}$ or $\Phi_{0}$ is a root system of type $A_{2}$.

Proof. Let $\beta \in \Phi_{0}$. If $\left\langle\beta, \alpha^{\prime}\right\rangle=0$, then $c_{\alpha} \beta \in \Phi(\mathfrak{m}, \mathfrak{t})$ and $\left\langle c_{\alpha} \beta, \lambda\left(h_{0}\right)\right\rangle=0$. But $h_{0} \in \mathscr{D}$ implies that $\lambda\left(h_{0}\right)$ is regular. Thus $\left\langle\beta, \alpha^{\prime}\right\rangle \neq 0$ for any $\beta \in \Phi_{0}$. Now $A_{1}$ and $A_{2}$ are the only root systems which contain a root with no roots orthogonal to it. Q.E.D.

Lemma 10.20. Let $\left(h_{0}, \nu_{0}\right) \in \mathscr{D} \times \mathscr{F}, \alpha \in \Phi_{R}^{+}$, such that $m_{\alpha}^{*}(h: \nu)$ is not jointly smooth at $\left(h_{0}, \nu_{0}\right)$. Then $\Phi_{0}=\left\{ \pm \alpha^{\prime}\right\}$.

Proof. By Lemma 10.19 either $\Phi_{0}=\left\{ \pm \alpha^{\prime}\right\}$ or $\Phi_{0}$ is of type $A_{2}$. Suppose $\Phi_{0}$ is of type $A_{2}$. Then $c^{-1} \Phi_{0}= \pm\left\{\alpha, \gamma, \gamma^{\sigma}\right\}$, where $\gamma, \gamma^{\sigma}$ are conjugate complex roots of $(\mathfrak{g}, \mathfrak{h})$ with $\gamma+\gamma^{\sigma}=\alpha$. One of ${ }^{c} \gamma,{ }^{c} \gamma^{\sigma}$, say ${ }^{c} \gamma^{\sigma}$, must be a 
compact root of $\left(\mathfrak{m}^{\prime}, \mathfrak{t}^{\prime}\right)$. Now $(\gamma, \nu)=\left\langle\gamma^{\sigma}, \nu\right\rangle=\frac{1}{2}\langle\alpha, \nu\rangle$ for all $\nu \in \mathscr{F}$ and $\left\langle\gamma^{\sigma}, \lambda(h)\right\rangle=-\langle\gamma, \lambda(h)\rangle$ for all $h$. Thus

$$
\begin{aligned}
\langle\gamma, \lambda(h)\rangle & =\frac{1}{2}\left\langle\gamma-\gamma^{\sigma}, \lambda(h)\right\rangle=\frac{1}{2}\left\langle{ }^{c} \gamma-{ }^{c} \gamma^{\sigma}, \lambda^{\prime}(h)\right\rangle \\
& =\frac{1}{2}\left\langle^{c} \gamma+{ }^{c} \gamma^{\sigma}, \lambda^{\prime}(h)\right\rangle=\frac{1}{2}\left\langle{ }^{c} \alpha, \lambda^{\prime}(h)\right\rangle
\end{aligned}
$$

for all $h$ since $\left\langle{ }^{c} \gamma^{\sigma}, \lambda^{\prime}(h)\right\rangle=\left\langle{ }^{c} \gamma^{\sigma}, \lambda^{\prime}\left(h_{0}\right)\right\rangle=0$ for all $h$. Thus

$$
\begin{aligned}
& \left\langle\lambda\left(h_{0}+h\right)+i\left(\nu_{0}+\nu\right), \gamma\right\rangle\left\langle\lambda\left(h_{0}+h\right)+i\left(\nu_{0}+\nu\right), \gamma^{\sigma}\right\rangle \\
& \quad=-\frac{1}{4}\left(\left\langle h,{ }^{c} \alpha\right\rangle^{2}+\langle\nu, \alpha\rangle^{2}\right)=C\left(h\left(-i Z_{\alpha}\right)^{2}+\nu\left(H_{\alpha}^{*}\right)^{2}\right)=C\left(h_{\alpha}^{2}+\nu_{\alpha}^{2}\right),
\end{aligned}
$$

where $C \neq 0$, and so $m_{\alpha}^{*}(h: \nu)$ is jointly smooth at $\left(h_{0}, \nu_{0}\right)$ by Lemma 10.5 . This contradicts our hypothesis. Q.E.D.

Suppose $\Phi_{0}=\left\{ \pm \alpha^{\prime}\right\}$. Then $\lambda^{\prime}\left(h_{0}\right)$ is semiregular. In particular, it is not orthogonal to any compact root. Thus both $\Theta\left(M^{\prime 0}: \lambda^{\prime}\left(h_{0}\right): \mathscr{C}^{\prime}\right)$ and $\Theta\left(M^{\prime 0}: \lambda^{\prime}\left(h_{0}\right): s_{\alpha^{\prime}} \mathscr{C}^{\prime}\right)$ are nonzero limits of discrete series. Hence $\Theta\left(H: \chi\left(h_{0}\right): \lambda\left(h_{0}\right): \nu_{0}\right)$ is reducible. We can assume $\mathscr{C}^{\prime}$ is chosen so that

$$
\lambda^{\prime}\left(h_{0}+\xi h_{1}\right) \in\left\{\begin{array}{ll}
\mathscr{C}^{\prime} & \text { if } \xi>0, \\
s_{\alpha^{\prime}} \mathscr{C}^{\prime} & \text { if } \xi<0
\end{array} \text { for } \xi\right. \text { sufficiently small }
$$

Thus,

$$
\begin{aligned}
\left(D_{h}^{\alpha}\right)^{k} & \Theta\left(H^{\prime}: \chi^{\prime}\left(h_{0}\right): \lambda^{\prime}\left(h_{0}\right): \mathscr{C}^{\prime}: \nu_{0}^{\prime}\right) \\
& =\lim _{\xi \downarrow 0}\left(\frac{d}{d \xi}\right)^{k} \Theta\left(H^{\prime}: \chi^{\prime}\left(h_{0}+\xi h_{1}\right): \lambda^{\prime}\left(h_{0}+\xi h_{1}\right): \nu_{0}^{\prime}\right)
\end{aligned}
$$

and

$$
\begin{aligned}
\left(D_{h}^{\alpha}\right)^{k} & \theta\left(H^{\prime}: \chi^{\prime}\left(h_{0}\right): \lambda^{\prime}\left(h_{0}\right): s_{\alpha^{\prime}} \mathscr{C}^{\prime}: \nu_{0}^{\prime}\right) \\
& =\lim _{\xi \uparrow 0}\left(\frac{d}{d \xi}\right)^{k} \theta\left(H^{\prime}: \chi^{\prime}\left(h_{0}+\xi h_{1}\right): \lambda^{\prime}\left(h_{0}+\xi h_{1}\right): \nu_{0}^{\prime}\right)
\end{aligned}
$$

are actual limits of derivatives along a continuous family of tempered characters.

Theorem 10.22. Let $\left(h_{0}, \nu_{0}\right) \in \mathscr{D} \times \mathscr{F}$ and $\alpha \in \Phi_{R}^{+}$such that $m_{\alpha}^{*}$ is not jointly smooth at $\left(h_{0}, \nu_{0}\right)$. Let $f \in \mathscr{C}(G)$. Then for all $k \geq 0, x \in G$,

$$
\begin{aligned}
&\left(D_{h}^{\alpha} \pm i D_{\nu}^{\alpha}\right)^{k} \Theta\left(H: \chi\left(h_{0}\right): \lambda\left(h_{0}\right): \nu_{0}: R(x) F\left(h_{0}\right)\right) \\
&=\left(\lim _{\xi \downarrow 0}+\lim _{\xi \uparrow 0}\right)\left(\frac{d}{d \xi}\right)^{k} \Theta\left(H^{\prime}: \chi^{\prime}\left(h_{0}+\xi h_{1}\right): \lambda^{\prime}\left(h_{0}+\xi h_{1}\right)\right. \\
&\left.\quad: \nu_{0}^{\prime}: R(x) F\left(h_{0}+\xi h_{1}\right)\right) .
\end{aligned}
$$

Proof. For any $f \in \mathscr{C}(G)$ and $h \in i \mathrm{v}^{*}, F(h) \in \mathscr{C}(G / Z, \zeta(h): W)$ is defined as in (4.2b). Now if $\Theta(h)$ is the character of any tempered representation with $Z$-character $\zeta(h)$ we have $\Theta(h: R(x) F(h))=\int_{\omega} \overline{\Theta(h: y)} F(h: y x) d y$, where $\omega$ is a fundamental domain in $G$ for $G / Z$. We can assume $\sigma_{V}$ is bounded 
on $\omega$ since $V / Z$ is compact. Assume for a moment that we can exchange the orders of integration and differentiation. Then

$$
\begin{aligned}
&\left(D_{h}^{\alpha} \pm i D_{\nu}^{\alpha}\right)^{k} \Theta\left(H: \chi\left(h_{0}\right): \lambda\left(h_{0}\right): \nu_{0}: R(x) F\left(h_{0}\right)\right) \\
&=\int_{\omega} \sum_{j=0}^{k}\left(\begin{array}{l}
k \\
j
\end{array}\right)\left(D_{h}^{\alpha} \pm i D_{\nu}^{\alpha}\right)^{j} \Theta\left(H: \chi\left(h_{0}\right): \lambda\left(h_{0}\right): \mathscr{C}: \nu_{0}: y\right) \\
& \quad \times\left(D_{h}^{\alpha}\right)^{k-j} F\left(h_{0}: y x\right) d y
\end{aligned}
$$

and

$$
\begin{gathered}
{\left[\begin{array}{l}
\left.\lim _{\xi \downarrow 0}+\lim _{\xi \uparrow 0}\right]\left(\frac{d}{d \xi}\right)^{k} \Theta\left(H^{\prime}: \chi^{\prime}\left(h_{0}+\xi h_{1}\right): \lambda^{\prime}\left(h_{0}+\xi h_{1}\right): \nu_{0}: R(x) F\left(h_{0}+\xi h_{1}\right)\right) \\
=\int_{\omega} \sum_{j=0}^{k}\left(\begin{array}{l}
k \\
j
\end{array}\right)\left(D_{h}^{\alpha}\right)^{j}\left[\Theta\left(H^{\prime}: \chi^{\prime}\left(h_{0}\right): \lambda^{\prime}\left(h_{0}\right): \mathscr{C}^{\prime}: \nu_{0}^{\prime}: y\right)\right. \\
\left.+\Theta\left(H^{\prime}: \chi^{\prime}\left(h_{0}\right): \lambda^{\prime}\left(h_{0}\right): s_{\alpha^{\prime}} \mathscr{C}^{\prime}: \nu_{0}^{\prime}: y\right)\right] \\
\times\left(D_{h}^{\alpha}\right)^{k-j} F\left(h_{0}: y x\right) d y
\end{array}\right.}
\end{gathered}
$$

by $(10.21)$, so the result follows from Proposition 10.18. To move the derivatives inside the integral we note that by Lemma 6.4, given any $D \in D\left(i v^{*}\right)$ there exist $t \geq 0$ and $S \subseteq \mathscr{U}(\mathfrak{g}) \oplus \mathscr{U}(\mathfrak{g})$ so that for all $s \geq 0, f \in \mathscr{C}(G), x \in G$,

$$
\sup _{h \in i 0^{*}}\|F(h ; D: y x)\| \leq \Xi(y x)(1+\sigma(y x))^{-s}\left(1+\sigma_{V}(y x)\right)^{t} \mu_{t+t_{0}+s, s}(f) \text {. }
$$

Now for $x \in G$ fixed, given $s \geq 0$, there is $C_{s}>0$ so that

$$
\sup _{h \in i 0^{*}}\|F(h ; D: y x)\| \leq C_{s} \Xi(y)(1+\sigma(y))^{-s} \text { for all } y \in \omega .
$$

Further, if $\Theta(h: \nu)$ is either of the induced representations

$$
\Theta(H: \chi(h): \lambda(h): \nu) \text { or } \Theta\left(H^{\prime}: \chi^{\prime}(h): \lambda^{\prime}(h): \nu^{\prime}\right)
$$

we have, as in Lemma 6.10, using the formula for induced characters given in $[6,3.1]$, that given any $D \in D\left(i v^{*} \times \mathscr{F}\right)$, there are $C, t \geq 0$ so that

$$
\sup _{h, \nu}|\Theta(h: \nu ; D: y)| \leq C|\Delta(y)|^{-1}(1+\tilde{\sigma}(y))^{t} \quad \text { for all } y \in G .
$$

Now for $y \in \omega$, there is $C \geq 0$ so that $(1+\tilde{\sigma}(y))^{t} \leq C(1+\sigma(y))^{t}$. Finally, as in $[6,2.15]$, we can pick $s>0$ large enough that $\int_{\omega} \Xi(y)|\Delta(y)|^{-1}(1+\sigma(y))^{t-s} d y<$ $\infty$. Q.E.D.

Remark 10.23. For $f \in \mathscr{C}(G)$ we have Fourier coefficients

$$
\widehat{F}(h: \nu: x)=\Theta(H: \chi(h): \lambda(h): \nu: R(x) F(h))
$$

and

$$
\widehat{F}\left(H^{\prime}: h: \nu^{\prime}: x\right)=\Theta\left(H^{\prime}: \chi^{\prime}(h): \lambda^{\prime}(h): \nu^{\prime}: R(x) F(h)\right)
$$


$h \in i^{*}, \nu \in \mathscr{F}, \nu^{\prime} \in \mathscr{F}^{\prime}=\left(\mathfrak{a}^{\prime}\right)^{*}$. Theorem 10.22 gives a matching condition between certain derivatives of these Fourier coefficients at the points $\left(h_{0}, \nu_{0}\right)$, where the $H$-and the $H^{\prime}$-series of representations meet. This is the type of matching condition that must hold in order that the sum of wave packets

$\int_{\mathscr{D} \times \mathscr{F}} \hat{F}(h: \nu: x) m(h: \nu) d h d \nu+\int_{\mathscr{D}^{\prime} \times \mathscr{F}^{\prime}} \hat{F}\left(H^{\prime}: h: \nu^{\prime}: x\right) m\left(H: h: \nu^{\prime}\right) d h d \nu^{\prime}$ is a Schwartz class function on $G$ when the individual wave packets are not Schwartz.

Theorem 10.24. Let $f \in \mathscr{C}_{H}(G)$. Then

$$
(h, \nu) \mapsto \Theta(H: \chi(h): \lambda(h): \nu:(R(x) F)(h)) m(h: \nu)
$$

is jointly smooth on $\operatorname{cl}(\mathscr{D}) \times \mathscr{F}$.

Proof. Let $\alpha \in \Phi_{R}^{+}$and $\left(h_{0}, \nu_{0}\right) \in \mathscr{D} \times \mathscr{F}$ such that $m_{\alpha}^{*}$ is not jointly smooth at $\left(h_{0}, \nu_{0}\right)$. By Theorem 10.12 it suffices to prove that

$$
\left(D_{h}^{\alpha} \pm i D_{\nu}^{\alpha}\right)^{k} \widehat{F}\left(h_{0}: \nu_{0}: x\right)=0 \text { for all } k \geq 0 .
$$

Now since $f \in \mathscr{C}_{H}(G)$,

$$
\Theta\left(H^{\prime}: \chi^{\prime}\left(h_{0}+\xi h_{1}\right): \lambda^{\prime}\left(h_{0}+\xi h_{1}\right): \nu^{\prime}: R(x) F(h)\right) m\left(H^{\prime}: h_{0}+\xi h_{1}: \nu^{\prime}\right)=0
$$

for almost all $\xi \in \mathbf{R}, \nu \in \mathscr{F}^{\prime}$. But $m\left(H^{\prime}: h_{0}+\xi h_{1}: \nu^{\prime}\right)$ is possibly zero or undefined only if $\left\langle\lambda^{\prime}\left(h_{0}+\xi h_{1}\right)+i \nu^{\prime}, \beta\right\rangle=0$ for some $\beta \in \Phi\left(\mathfrak{g}_{\mathbf{C}}, \mathfrak{h}_{\mathbf{C}}^{\prime}\right)$. But $\lambda^{\prime}\left(h_{0}+\xi h_{1}\right)+i \nu^{\prime}$ is regular for almost all $\left(\xi, \nu^{\prime}\right) \in \mathbf{R} \times \mathscr{F}^{\prime}$. Thus, since it is smooth,

$$
\Theta\left(H^{\prime}: \chi^{\prime}\left(h_{0}+\xi h_{1}\right): \lambda^{\prime}\left(h_{0}+\xi h_{1}\right): \nu^{\prime}: R(x) F\left(h_{0}+\xi h_{1}\right)\right)=0
$$

for all $\left(\xi, \nu^{\prime}\right) \in \mathbf{R} \times \mathscr{F}^{\prime}$. Thus using Theorem 10.22,

$$
\left(D_{h}^{\alpha} \pm i D_{\nu}^{\alpha}\right)^{k} \Theta\left(H: \chi\left(h_{0}\right): \lambda\left(h_{0}\right): \nu_{0}: R(x) F\left(h_{0}\right)\right)=0
$$

for all $k \geq 0$. Q.E.D.

\section{REFERENCES}

1. Harish-Chandra, Harmonic analysis on real reductive groups. I, J. Funct. Anal. 19 (1975), 104-204.

2. __ Harmonic analysis on real reductive groups. II, Invent. Math. 36 (1976), 1-55.

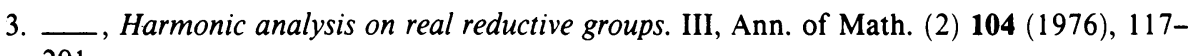
201.

4. R. A. Herb, The Schwartz space of a general semisimple Lie group. III: c-functions, Adv. in Math. (to appear).

5. R. A. Herb and J. A. Wolf, The Plancherel Theorem for general semisimple groups, Compositio Math. 57 (1986), 271-355.

6. __ Rapidly decreasing functions on general semisimple groups, Compositio Math. 58 (1986), 73-110.

7. Wave packets for the relative discrete series I: The holomorphic case, J. Funct. Anal. 73 (1987), 1-37. 
8. _ Wave packets for the relative discrete series II: The non-holomorphic case, J. Funct. Anal. 73 (1987), 38-106.

9. __ The Schwartz space of a general semisimple Lie group I: Wave packets of Eisenstein integrals, Adv. in Math. 80 (1990), 164-224.

10. H. Kraljević and D. Miličić, The $C^{*}$-algebra of the universal covering group of $S L(2, \mathbf{R})$, Glas. Mat. Ser. III 7 (27) (1972), 35-48.

11. J. Mather, Stable $C^{\infty}$ mappings I: The division theorem, Ann. of Math. (2) 87 (1968), 89-104.

12. W. Schmid, Two character identities for semisimple Lie groups, Non-Commutative Harmonic Analysis (Marseille-Luminy 1976), Lecture Notes in Math., vol. 587, Springer-Verlag, New York, 1977.

13. J. A. Wolf, Discrete groups, symmetric spaces, and global holonomy, Amer. J. Math. 84 (1962), 527-542.

14. _ Homogeneity and bounded isometries in manifolds of negative curvature, Illinois $\mathrm{J}$. Math. 8 (1964), 14-18.

15. _ Unitary representations of partially holomorphic cohomology spaces, Mem. Amer. Math. Soc. No. 138 (1974).

Department of Mathematics, The University of Maryland, College Park, Maryland 20742 